\title{
Animal models of cognitive aging and dementia in the rat : problems and perspectives
}

Citation for published version (APA):

Blokland, A. (1992). Animal models of cognitive aging and dementia in the rat : problems and perspectives. [Doctoral Thesis, Maastricht University]. Datawyse / Universitaire Pers Maastricht. https://doi.org/10.26481/dis.19920918ab

Document status and date:

Published: 01/01/1992

DOI:

10.26481/dis.19920918ab

Document Version:

Publisher's PDF, also known as Version of record

\section{Please check the document version of this publication:}

- A submitted manuscript is the version of the article upon submission and before peer-review. There can be important differences between the submitted version and the official published version of record.

People interested in the research are advised to contact the author for the final version of the publication, or visit the DOI to the publisher's website.

- The final author version and the galley proof are versions of the publication after peer review.

- The final published version features the final layout of the paper including the volume, issue and page numbers.

Link to publication

\footnotetext{
General rights rights.

- You may freely distribute the URL identifying the publication in the public portal. please follow below link for the End User Agreement:

www.umlib.nl/taverne-license

Take down policy

If you believe that this document breaches copyright please contact us at:

repository@maastrichtuniversity.nl

providing details and we will investigate your claim.
}

Copyright and moral rights for the publications made accessible in the public portal are retained by the authors and/or other copyright owners and it is a condition of accessing publications that users recognise and abide by the legal requirements associated with these

- Users may download and print one copy of any publication from the public portal for the purpose of private study or research.

- You may not further distribute the material or use it for any profit-making activity or commercial gain

If the publication is distributed under the terms of Article $25 \mathrm{fa}$ of the Dutch Copyright Act, indicated by the "Taverne" license above, 
ANIMAL MODELS OF COGNITIVE AGING AND DEMENTIA IN THE RAT:

PROBLEMS AND PERSPECTIVES 



\section{ANIMAL MODELS OF COGNITIVE AGNG AND DEMENTIA IN THE RAT: PROBLEMS AND PERSPECTIVES}

\section{PROEFSCHRIFT}

ter verkrijging van de graad van doctor aan de Rijksuniversiteit Limburg te Maastricht, op gezag van de Rector Magnificus, Prof. Mr. M.J. Cohen, volgens het besluit van het College van Dekanen, in het openbaar te verdedigen op vrijdag, 18 september 1992 om 14.00 uur

door

\section{ARJAN BLOKLAND}

geboren op 30 januari 1962 te Heerlen 
PROMOTORES:

Prof. Dr. J. Jolles

Dr. W.G.M. Raaijmakers

\section{BEOORDELINGSCOMMISSIE:}

Prof. Dr. H.A.J. Struijker Boudier (voorzitter)

Dr. M. de Baets

Prof. Dr. W.H. Gispen (Rijksuniversiteit van Utrecht)

Dr. J. Lodder

Dr. H. Merckelbach

Prof. Dr. N.E. van de Poll (Nederlands Instituut voor Hersenonderzoek)

ISBN 90-5278-033-1

The printing of the thesis was financially supported by Ethifarma Nederland B.V., a Sigma Tau Company. 


\section{CONTENTS}

Chapter 1: General Introduction

Introduction

Aspects of age-related cognitive decline

Animal models of the cognitive decline in aging

Comparison of age groups in learning and memory tasks

Treatments for cogritive aging and dementia 5

The objectives of the present study

Summary of experiments

Chapter 2: The cone field: a spatial discrimination task for the automatic and simultaneous assessment of working and reference memory in the rat.

Abstract

Introduction

Experiment 1: Spatial discrimination performance of young and senescent female BN rats

Materials and methods

Results

Discussion

Experiment 2: The cone field as a task for the longitudinal assessment of spatial discrimination learning

Materials and methods

Results

Discussion

General Discussion

Chapter 3: Different time course for age-related changes of behavior in a complex spatial cone field discrimination task in Lewis rats

Abstract

Introduction

Materials and methods

Results

Discussion

Chapter 4: Spatial discrimination learning and orientation strategy in young and old Lewis rats

Abstract

Introduction

Materials and methods

Results

Discussion.

Chapter 5: Age-related changes in spatial discrimination performance in Lewis rats

Abstract

Introduction 
Experiment 1: Acquisition of the standard: Morris task in Lewis rast of three different ages

Matertals and method

Results and conclusions.

Experiment 2: The performatnce of Lewis ratts of three different ages in a learning set paradigm

Materials and method

Results and conclusions

Experiment3: The performance of young and old Lewis rats in the water escape task with a fixed start and platform position

Materials and method

Results and conclusions

Experiment 4: The effects of introducing proximal cues to the testing environment in young and old rats

Materials and method

Results and conclusions

General discussion

Chapter 6: Age-related changes in the response to stressful stimuli in different rat strains

Abstract

Introduction

Materials and methods

Results

Discussion

Chapter 7: Age differences in food motivation in Brown-Norway rats

Abstract

Introduction

Materials and methods

Results

Discussion

Chapter 8: Correlation between behavioral and biochemical parameters in young and old Lewis rats

Abstract

Introduction

Materials and methods

Results

Discussion

Chapter 9: Spatial learning deficit and reduced hippocampal ChAT activity after an i.c.v. injection of streptozotocin

Abstract

Introduction

Materials and methods

Results

Discussion 
Chapter 10: Differential effect of acefyl-1-carnitine on open field behavior in young and old rats

Abstract 91

Introduction

Materials and methods

Results and discussion

Chapter 11: Neuroprotective effects of acetyl-1-carnitine in a new paradigm for behavioral assessment during acute metabolic inhibition

Abstract

Introduction

Materials and methods

Resulls

Discussion

Chapter 12: Summary and conclusions

Introduction

Summary of studies

Conclusions

Summary of conclusions

References

Samenvatting en conclusies

Dankwoord 



\section{GENERAL INTRODUCTION}

\section{Introduction}

Over the last century the lifespan of people has increased and many people in western couniries now live to become older than 60 years of age. This demographic development, which has also been referred to as double aging (dubbele vergrijzing), has made age-related problems and diseases more prominent. The elderly frequently complain of a decline in cognitive functions especially in functions related to remembering and forgetting. The cognitive problems may be summarized as a decline in remembering events, a general slowing of behavior, and a decreased efficiency in problem-solving tasks (for review see Birren \& Schaie, 1985). Recently, it has been shown that the agerelated decline in cognitive functions is also related to neurological and psychiatric abnormalities, and biological life events (Crook et al., 1986; Houx, 1991). Although there may be slight differences between individuals in the degree of the cognitive decline, it affects almost everyone eventually. The most important consequence of this cognitive decline is that it affects the quality of life. People may see themselves as not being competent to participate in daily life, which may lead to symptoms that are secondary to the cognitive impairments, e.g., depression and anxiety.

Even more dramatic than the normal process of cognitive aging is the progressive disease dementia. In the Diagnostic Statistical Manual of the American Psychiatric Association, dementia is defined as a progressive clinical state which is characterized by a deterioration of intellect, memory, judgement, and abstract thinking (1987). In later stadia, people suffering from dementia will become dependent on the help of others. It has been estimated that approximately 10 percent of the population over 60 years of age will be affected by dementia, of which the most prevalent form is dementia of the Alzheimer's type.

The primary aim of current aging research is to understand the process of aging and to elucidate the possible causes of the age-related cognitive decline. This research should yield: 1) treatments which attenuate the cognitive disturbances in aging and dementia, 2) diagnostic tools to recognize early stages of abnormal aging, and 3) information which may help prevent the development of the cognitive decline in aging and dementia. With respect to this last point, it has recently been found that there are risk factors (biological life events) which, for a substantial part, account for the cognitive decline in aged people (see Houx, 1991). Consequently, this research may improve the quality of life of aged people by alleviating their poor cognitive functioning. Before this goal can be reached, a better understanding of the aging process is required. At present, however, the processes which underlie the manifestation of cognitive aging and dementia are still poorly understood. 


\section{Aspects of age-related cognitive decline}

Human studies have shown that aging affects performance in various tasks o a broad range of cognitive functions (for more extensive reviews see Botwimick, 1981; Jolles, 1986; Light, 1991; Salthouse, 1988). The overall finding that old subjects never perform better than their younger controls on memory tasks, i.e., no cross-over interaction, suggests that a (one or more) fundamental process underlies the memory deficit (Light, 1991). It should, however, be noted that several studies have shown that older subjects perform better on particular verbal tasks. Thus, although there is a general decline in cognitive functions, it seems that there is a difference between the magnitude of the decine in the various cognitive functions. Well-trained skills and knowledge seem to be preserved in aged people. Also, when new material has to be remembered passively (recognition of new material), aging seems to have only a minor effect. The most prominent age-related deficits are observed when new material has to be used actively (encoding and retrieval of new information) (Jolles, 1986).

Another aspect of age-related changes in cognition is a general slowing of information-processing (Salthouse, 1985; 1990). It is as yet, however, unclear what peripheral and/or central mechanisms underlie this well-known phenomenon. The slowing of information processing is assumed to contribute to the age-related decline in cognitive processes. For example, the slower rate of processing may account for the age-related deficits found in tasks in which one has to deal with an information overload or when one has to switch between different information entities, such as selective attention. The cognitive slowing may also lead to age-related changes in the use of problem-solving strategies (Salthouse, 1985). It has also been suggested that older people are more rigid in their behavior and more likely to make perseveration errors than young subjects (see Dean \& Bartus, 1988).

Many studies have been conducted to characterize the age-related cognitive decline in which different learning and memory tasks were used. However, many studies in which cognitive functions were assessed in old and young subjects did not control sufficiently for confounding factors (e.g. motivation. health, sensory/motor function) and/or methodological problems (e.g., selection of subjects) (see Dean \& Bartus, 1988; Houx, 1991). Consequently, the nature of the age-related cognitive changes remains poorly defined. However, there is a growing body of evidence to suggest that some tasks are especially sensitive to the effect of aging. Aged subjects seem to have problems with remembering recent events whereas immediate and long term memory are practically unaffected. The extent of the age-related deficit in recent memory increases with the complexity of the material to be remembered. Furthermore, there is circumstantial evidence that aged people are more sensitive to interfering stimuli than younger subjects. However, al though some characteristics of the age-related cognitive decline have emerged, more studies which control for confounding factors and which take methodological issues into account are needed to understand the cause and nature of the decline in cognitive functions with increasing age. 


\section{Animal models of the cognitive decline associated with aging}

Because the use of humans in aging studies has ethical and practical restrictions, animals have become important in investigating the age-related decline in cognitive functions. The advantages of using animals instead of humans in aging research are numerous (e.g., control of possible confounding variables, access to brain tissue, control of genotype, shorter life span). However, the major problem of animal models is the external validity: is the aged animal comparable to the aged human. Moreover, findings from studies with animals do not resemble those characteristic of human disease states (Gamzu, 1985). Although the typical features of the decline in cognitive functions may differ for man and animals, the agreement between the nature of the behavioral deficits in aged subjects of several species (see below) may suggest that a common neurological etiology may underlie the deficits (Dean \& Bartus, 1988). In a special issue of the journal Neurobiology of Aging' (volume 9, 1988, pages 441-742), attention was given to animal models of aging. Most authors favored the use of animal models for the age- and disease-related decline in cognitive functions, although each one raised doubts with respect to a certain topic. One of the topics was what paradigm could be used in animal studies that could have a predictive value for humans.

Virtually all studies which have evaluated the age-related decline in cognitive functions have used learning and memory tasks. However, old animals do not show an impaired performance on every task when compared to young controls. In rats, it has been found that learning and memory tasks have to have certain features to reveal an age-related performance deficit. Goodrick (but also Doty $(1966,1972))$ may be seen as a pioneer in evaluating the task aspects which are required to reveal age-related learning and memory deficits in rats. Two important task aspects are: 1) complexity (Goodrick, 1972), and 2) distribution of practice (Goodrick, 1973). The more complex a task is and the less trials given per day, the greater the age-related perlormance deficit. Furthermore, Goodrick suggested that old rats are behaviorally more rigid than young rats (Goodrick. 1975) and that a decline in short-term memory processes could underlie the performance deficits in complex mazes (Goodrick, 1980). These task requirements resemble those that have been used in human research (see above) and indicate that there is a similarity in the nature of cognitive aging between species. Thus paradigms for studying the age-related cognitive decline should recognize the task requirements mentioned above. Learning and memory tasks should have at least one characteristic which is known to be sensitive to aging. Another important point is that age-related cognitive deficits observed in animals should show a similarity to age-related cognitive deficits found in man. Two paradigms which have consistently revealed age-related performance deficits that are comparable to findings from human studies are: 1) short-term memory tasks, and 2) spatial discrimination learning tasks.

In one of two paradigms considered to be useful as an animal model of 
cognitive aging, the performance of animals is assessed in short-term memory tasks. In short-term memory tasks animals hawe to remember a response or a stimulus in order to make a correct response after a delay. Numerous studies have shown an age-related performance deficit in these tasks in the rat (e.g. Dunnett et al., 1988; Hamm et al., 1983). However, it seems that the complexity of the information to be held in short-term memory may be important in determining whether an age-related performance deficit is observed (Wallace et al., 1980; Willig et al., 1987). This is in line with the findings of Goodrick (1972 „see above). The length of the delay is also a critical aspect for detecting an age effect (Dunnett et al,, 1988). The deficit in short-term memory processes has been described as an increased rate of forgetting with aging (see Willig et al., 1987). Similar deficits have also been observed in humans (Craik, 1977; Flicker et al., 1985). The resemblance between humans and animals may indicate that shortterm memory tasks can be used to evaluate the age-related cognitive decline.

The other paradigm in which a consistent age-related decline in performance has been found in the rat is spatial discrimination learning (e.g., Gage et al., 1984a; Gallagher \& Pelleymounter, 1988b; Rapp et al., 1987). In spatial discrimination tasks animals can make the correct response(s) by making use of stimuli from the environment. There are different ways of moving to an object in space (see Kollb \& Whishaw, 1990). The first possibility is to use the body as a reference for subsequent movements. No external cues are required with this kind of spatial orientation, which is also referred to as "egocentric orientation". Furthermore, one can make use of a specific cue in the environment and move towards or away from that specific environmental cue. The cue is essential in this type of spatial orientation. In the third type of spatial orientation, also referred to as "allocentric orientation", the relationship among different environmental cues guides one's behavior. It will be clear that information processing is very complex in allocentric orientation, because the relation among different stimuli has to be calculated. The age-related performance deficit in allocentric orientation tasks is assumed to be caused by the complexity of processing the spatial information. An age-related deficit in allocentric orientation has also been reported in humans (see Gallagher \& Pelleymounter, 1988b; Olton, 1988). Tasks in which an allocentric orientation strategy is used may therefore be regarded as a useful paradigm for studying the age-related cognitive decline.

One animal model that has received much attention is the nucleus basalis lesion model. This nucleus degenerates in Alzheimer's disease (Whitehouse et al., 1981). Numerous studies have evaluated the effects of nucleus basalis lesions and found profound deficits in short-term memory and spatial discrimination tasks in the lesioned animals (Dunnett, 1985; Hepler et al., 1985; Whishaw et al., 1985). The correlation between a decreased cortical cholinergic activity and learning and memory impairments suggested that this paradigm could be a valuable and rather simple model of dementia. However, the cognitive dysfunction observed in the nucleus basalis lesion model in the rat was recently suggested not to resemble the cognitive dysfunction in Alzheimer's disease (Dunnett et al., 1991). The nucleus basalis lesion model nicely demonstrates the 
methodological problems associated with animal models (for review see Dunnett et al., 1991). One of the problems with the nucleus basalis lesion model is that the clustering of cholinergic cells in one nucleus, as found in man (the nucleus basalis of Meynert) is not observed in rodents (Salamone, 1986). As a consequence, lesioning of the nucleus basalis in rats may also damage other cells which have no cholinergic output to the cortex (e.g., neurons in the globus pallidus), and this may complicate the interpretation of results in terms of a decrease in centrall cholinergic functions.

\section{Comparison of age groups in learning and memory tasks}

As mentioned above, the difference in performance between young and old subjects has predominantly been investigated in learning and memory tasks. However, when considering the definition of learning formulated by Bower and Hilgard (1981),

"Learning refers to the change in a subject's behavior or behavioral potential to a given situation brought about by the subject's repeated experiences in that situation, provided that the behavioral change cannot be explained on the basis of the subject's native response tendencies, maturation, or temporary states (such as fatigue, drunkenness, drives and so on)."

it could be argued, with respect to this definition, that age-related differences in a change in behavior can be explained in terms of an age-related difference in native response tendency, maturation, fatique etc. Consequently, learning may not be comparable for young and old subjects. Many authors have described the problems associated with aging studies and the consequences of these problems for the interpretation of behavioral data (e.g., Ingram, 1985; Ray \& Barrett, 1973; Rigter, 1983; Sarter, 1987). Possible factors that could confound results obtained from aging studies are age-related differences in, for example, motivation, speed of responding, reactivity to stress, sensory sensitivity, motor performance, attentional processes, problem-solving strategies, exploration. Although the pitfalls (at least those that have been recognized) have been distinguished, it has often been concluded from aging studies that there are age-related differences in learning and memory without possible confounding factors having been controlled for. Because learning is a result of multiple (non)cognitive processes, a first step should be to identify the processes that play a crucial role in the learning process. Once the major processes have been recognized, it should be possible to obtain a better understanding of the cause and nature of the age-related performance deficits.

\section{Treatments for cognitive aging and dementia}

It is well established that the integrity of the central cholinergic system plays an important role in learning and memory processes (see Hagan \& Morris, 1988) 
The cholinergic hypothesis of geriatric memory dysfunction claims that the agerelated decline in cognitive function is largely related to the deterioration of central cholinergic function (Bartus et al., 1982, 1985). There are two different observations which support the hypothesis. The first observation is that cholinergic markers have been found to be decreased in aging and dementia (McGeer \& McGeer, 1978; Perry, 1980). The nucleus basalis of Meynert, which has a cholinergic projection to the cortex, is degenerated in patients suffering from dementia of the Alzheimer type (Whitehouse et al., 1981) and the destruction causes a dramatic decrease in central cholinergic markers (Bowen et al., 1976). The parallel between the decrease in central cholinergic function and cognitive deficits (Katzman, 1986) strongly supports the cholinergic hypothesis of geriatric memory dysfunction. A second observation that supports the cholinergic hypothesis is that cholinergic antagonists cause memory deficits in young subjects (Broks et al., 1988; Drachman \& Leavitt, 1974).

However, one requirement which should also be met in the cholinergic hypothesis, namely that an enhancement of central cholinergic activity should ameliorate the age-related cognitive impairments, has yielded contradictory results and clinical findings have been largely negative (for reviews see Bartus et al., 1985; Collerton, 1986). Taken together, these findings suggest that the deterioration of the cholinergic system underlies the decline in learning and memory processes with aging but that it is questionable whether enhancement of the cholinergic system is able to attenuate the age-related cognitive decline (Fibiger, 1991).

Circumstantial evidence has been found that different classes of drugs which have no direct effect on the cholinergic system can attenuate the cognitive decline in aging (see Hock, 1987; Jolles, 1983; Sarter, 1991). Many different classes of drugs have been used in clinical and in animal studies, for instance, catacholaminergic drugs, hormones, neuropeptides, stimulants, cerebral vasodilatators, and opioids. Recently, the so-called nootropics have been suggested to alleviate cognitive functions (see Giurgia et al., 1983) and similar findings have been reported for calcium entry blockers (Levere \& Walker, 1991; Schuurman \& Traber, 1989). However, the development of different drugs of the classes mentioned above has not yielded a panacea for the cognitive decline in aging. A possible explanation for the disappointing results in the development of cognition-enhancing drugs could be that memory is not a unitary process and depends on different cerebral structures (Jolles, 1983). A better understanding of the pharmacology of memory could lead to drugs which improve cognitive function. Another explanation for the ineffectiveness of drugs could be that a more fundamental biochemical process causes the cognitive deficits. The decline in cholinergic function could be secondary to these more fundamental

\section{The objectives of this thesis}

From the introduction above it can be concluded that cognitive aging is still a 
poorly understood phenomenon at both the cognitive and biochemical level. The use of animals to explore the possible mechanisms underlying the agerelated decline in cognitive processes may at first sight be regarded as a very useful tool. However, behavioral data from animal aging studies are difficult to interpreted in terms of age-related differeneces in learning and memory processes. Both the internal and external validity of animal aging studies may be violated since, respectively, the performance of young and old subjects may be differently affected by confounding factors and the age-related deficits in rats may not be comparable to human deficits. The latter problem can be overcome by selecting an appropriate animal model. However, in order to control for possible confounding factors one should try to identify the nature of the age-related differences in performance in the learning and memory tasks. If in a learning and memory task the performance of young and old rats differs, one can, for example, come to the conclusions that there is a shift in the use of problem-solving strategies with age, or that there are age-related differences in unlearned behavioral respons tendencies which interfere with learning and memory performance. The age-related shift in the use of strategies can be the result of maturation or the loss of (non)cognitive abilities necessary to use a strategy. It will be clear that one has to be careful in drawing conclusions from studies in which learning and memory are assessed in young and old rats. One way to circumvent this problem is to try to control for factors that could interfere differently with learning and memory in young and old rats and to evaluate the behavior of young and old rats in more detail.

Confounding factors that may cannot be evaluated in a single experiment. The performance of young and old rats should preferentially be evaluated in different tasks which are assumed to measure one dimension of learning and memory. The use of different procedures in these learning tasks could also reveal information about how young and old rats solve a task. There are only a few studies which have related different aspects of behavior with age-related differences in learning and memory (e.g., Gage et al., 1989; Sprangler et al., 1989; Willig et al.,1987). The performance of young and old rats is usually assessed in only one task.

One of the aims of this dissertation is to illustrate that a better understanding of the nature of the age-related performance deficit in learning and memory tasks can be obtained by using different tasks and different procedures. In almost all of the experiments described in this dissertation the behavior of one strain of rats was used to exclude possible strain differences which may complicate the interpretation of the different behavioral tests. Although this may limit the generalization to other strains of rats, it is more appropriate for understanding the nature of the age-related cognitive/performance deficits of rats in learning tasks. Furthermore, the performance of rats of different ages was predominantly evaluated in spatial discrimination learning. The advantage of this is that it allows the evaluation of learning and memory performance of young and old rats in one dimension. A disadvantage of this is comparable to the one mentioned with the use of one strain, i.e., generalization. 
At present there are no adequate animal models in which a treatment/lesion causes behavioral deficits in animals comparable to deficits observed in human aging and dementia. The nucleus basalis model was until recently assumed to be a simple and valid animal model that could account for the cognitive deficits of aging and dementia. However, at present there are doubts whether this may be a valid model (see above). The finding that peptidases and phosphatidylinositol kinase are affected in Alzheimer's disease (Jolles et al., 1992; Terwel et al., 1992), may suggest that fundamental biochemical processes underlie the degeneration of neurons in pathological aging. It would therefore be interesting to evaluate whether disturbances in fundamental biochemical processes could result in cognitive impairments. This could lead to a better understanding of the processes that underlie the cognitive decline in (pathological) aging and consequently may lead to treatments which attenuate these biochemical processes. Another aim of this dissertation was to evaluate a recently developed animal model of dementia which has been characterized mainly at the biochemical level up till now. In this model the glucose uptake of neurons is disturbed after an i.c.w. injection of streptozotocin and this leads to biochemical changes comparable to those observed in dementia (Ding et al., 1992; Mayer et al., 1990; Nitsch et al, 1989). It was evaluated whether streptozotocin-treated rats showed an impaired performance in an animal model of aging.

As mentioned above, the decreased uptake of glucose in neurons may underlie the cognitive deficits of (pathological) aging. It will be clear that such a disturbance leads to a lower brain energy metabolism. A lower brain energy metabolism has been observed in both aging and dementia and has been found to correlate with cognitive function in man as well as in animals (see Gage et al., 1984b). Thus a lower brain energy metabolism may underlie the cognitive decline of aging and dementia. Finally, the effects of a drug known to influence energetic parameters were assessed. To test the hypothesis that this substance acts on the brain via changes in energy metabolism, a new behavioral paradigm was developed in which the cerebral energy metabolism was decreased experimentally.

\section{Summary of experiments}

The studies described in this dissertation attempted to identify (non)cognitive processes which may change during aging in the rat in order to get a better understanding of the age-related cognitive decline observed. In order to do this, we evaluated behavior in different learning and memory tasks with different procedures (chapters 1, 2, 3 and 4), evaluating non-cognitive processes 1 (chapters 5 and 6) and a within-subjects design (chapter 7). By careful examination of the

1 It should be mentioned here that (non)cognitive processes are assessed in tasks in which also cognitive processes are involved. However, the operationalized measure of a non-cognitive process 
results of the different behavioral experiments, it should be possible to understand what behavioral changes accompany the aging process. These experiments should reveal whether the behavior of young and old rats can be compared, i.e., whether the same cognitive processes underlie the performance of young and old rats in the learning tasks. One study (chapter 8 ) evaluated an animal model of the age-related decline in cognitive functions. In this model a fundamental process that could underlie the neuronal aging process was affected, and the behavioral and biochemical consequences of the intervention were correlated. Finally, two studies (chapters 9 and 10) evaluated the behavioral effects of a nootropic drug and an attempt was made to understand the mechanism of action of the drug. 



\title{
THE CONE-FIELD: A SPATIAL DISCRIMINATION TASK FOR THE AUTOMATIC AND SIMULTANEOUS ASSESSMENT OF WORKING AND REFERENCE MEMORY IN RATSI
}

\author{
F. Josef van der Staay, Brigitte Krechting, Arjan Blokland \\ and Wijnand Raaijmakers
}

\begin{abstract}
A cone-field apparatus was developed in which spatial working memory (WM) and reference menory (RM) can be assessed automatically and simultaneously. The cone-field is a large open field with 16 cones in it. A configuration of four cones was baited with a food pellet. In the holeboard, the predecessor of the cone-field, no clear criteria exist to distinguish between accidental and directed orientations toward the target (hole). In the cone-field the visit to the target (cone) is unambiguously defined. We found that senescent rats were impaired on both the WM and the $\mathrm{RM}$. The development of a fixed pattern of visiting the baited cones was effectively prevented by starting the rats randomly from four different positions. When only one start position was used, rats developed a fixed pattern of visiting the baited cones. "This pattern was more pronounced in the young than in the senescent animals. In the last experiment we explored whether the task might be suited for the assessment of WM and RM performance in longitudinal studies. Rats performed like naive animals on both memory components when re-acquisitions were separated by 4-month intervals. without training.
\end{abstract}

\section{Introduction}

The ability of rats to find their way through complex mazes has been known since the early beginnings of experimental animal psychology (cf. Munn, 1950). Since then a variety of mazes, each with its own characteristics, have been developed. Two major classes of mazes can be distinguished: 'sequential choice' mazes and "free choice" mazes (Lachman \& Brown, 1957).

A 'sequential choice' type maze consists of a fixed start position, and one correct route (with blind alleys to avoid) to a fixed goal (e.g., the Stone maze, Goodrick, 1968, 1975; Ingram, 1988). The animal learns a 'route' or a sequence of left/right choices in order to reach the goal. In a 'free choice' type maze food can be present at various locations in the apparatus, and the animal is free to visit these places in whichever order it wants to. A much used example of this type is the radial maze with eight or more arms radiating from a central platform (Olton \& Samuelson, 1976). Depending on the type of maze used, the most efficient behavior might either consist of using a strategy (running a fixed route or systematic scanning of the places that might contain a food reward) or to remember the list of places already visited during a trial.

Olton and Samuelson (1976) suppose that the latter happens in their 'elevated

1 This Chapter has been published in Journal of Neuroscience Methods, 31 (1990) 13-22 
radial maze' and that the list of visits is held in the 'working memory" (WM). Contrary to the WM, which holds information that is relevant only within a specific trial, the 'reference memory' (RM) (Olton \& Papas, 1979) holds trial independent information, for example about the locations where the food is hidden. Thus, if food can only be found in a subset of potential places that might be visited by the foraging rat, then two memory components can be distinguished: WM and RM.

Oades and Isaacson (1978) developed a hole board apparatus, in which both WM and RM can be assessed simultaneously. However, one problem with the holeboard task appeared to be the definition of a hole visit, as no clear criterion exists to distinguish between directed and accidental orientations toward a hole. Slightly different definitions may give rise to different results (cf. van Luijtelaar et al., 1989). This problem may place constraints on the value of the holeboard. Therefore, we developed a cone-field apparatus on the basis of the holeboard, in which visits to the baited and unbaited places are defined unambiguously. In addition, in the cone-field apparatus, tracing of visits is fully computerized. Thus, experimenter induced errors are excluded.

We are especially interested in age-related impairments in learning and memory. According to Barnes (1979), spatial discrimination tasks could serve as valuable experimental paradigms to study the cognitive and neurobiological changes which accompany aging. To be considered as a valid tool in aging research, conefield tasks should be sensitive to these naturally occurring declines.

Age-associated decreases in learning ability and memory performance have been found in 'sequential choice' mazes as well as in 'free-choice' type mazes, such as the circular platform and the radial maze (Barnes, 1979; Barnes et al., 1980; Ingram et al., 1981; Wallace et al., 1980), the Morris water maze (Gage et al., 1984a), and the holeboard (van der Staay et al., 1986, 1988).

We found that the WM and RM performance of aged animals in the holeboard was less good than that of young animals (van der Staay et al, 1986, 1988). Young rats, however, developed a more pronounced pattern of visiting the baited holes (fixed food-search pattern) in the course of training than old rats did (van der Staay et al., 1986). The development of a fixed food search pattern may comprise an effective way to solve the spatial discrimination and to reduce WM and RM errors. Such a response strategy however, could interfere with the interpretation of spatial discrimination performance in terms of WM and RM processes (Olton, 1988). Therefore, we payed special attention to this problem when developing a spatial discrimination task in the cone-field.

In this paper we describe experiments performed to develop and validate the cone-field as an apparatus in which various aspects of the spatial discrimination behavior of rats can be assessed. In the first experiment the spatial discrimination performance of young and senescent rats was compared. We examined the effect of randomizing the start position between trials on the development of a fixed
food-search pattern.

A second approach in validating the cone-field task consisted of investigating the effects of bilateral fimbria lesions on spatial discrimination performance 
(fully reported elsewhere: van der Staay et al, 1989), because it has been reported earlier that this type of lesions impair both the WM and the RM performance (cf. Jarrard, 1980). The possibility that the cone-field could be useful in longitudinal studies was explored using the intact control rats of the lesion experiment.

Experiment I: Spatial discrimination performance of young and senescent female BN rats

The aim of this experiment was to assess whether senescent animals show an age-related impairment of spatial discrimination performance in the cone-field apparatus. In addition, we investigated whether randomization of the start position might prevent the development of a fixed food-search pattern and whether the results with respect to WM and RM would be comparable to those found in the holeboard task (van der Staay et al, 1986; 1988).

\section{Material and methods}

\section{Animals}

Eight young (3-month-old) and nine senescent (33-rnonth-old) female Brown Norway (BN/BiRij) rats were supplied by the TNO Institute for Experimentall Gerontology, Rijswijk, the Netherlands. They were housed individually in standard MakrolonTM cages with sawdust bedding in an air conditioned room (ca. $20^{\circ} \mathrm{C}$ ). The lights were on from 7:00 to 19:00.

\section{Apparatus}

The cone-field was a dodecagonal open field with 16 cones in it and with four start boxes connected to it (see Fig. 1 for details and dimensions). The $45 \mathrm{~cm}$ high side walls were made of transparent poly(vinyl chloride). The floor consisted of stainless steel. Sliding doors, which gave access to the cone-field, were operated by the experimenter.

A food cup was placed in the top of each cone. One 45-mg foodpellet could be fixed in the centre of each food cup such that it could not be reached by the rat. These pellets made it impossible for the rat to detect the baited cones by means of odour cues emanating from the food cups. One $45 \mathrm{mg}$ food pellet was placed in each baited cone as a reward which the rat could obtain.

A visit to a cone is unambiguously operationally defined as a leaning response against a collar on the top of a cone. This response closes an electrical circuit between the collar and the floor and activates a counter. Inspection of the contents of the food cup and attainment of food pellets are conditional upon this leaning response. Only contacts preceded by a visit to another cone are scored (exception: the first cone visit). Infrared photocells detect whether a rat poked its nose into a food cup. An Apple//e microcomputer collected the data and controlled the duration of trials (in latter experiments, a MS-DOS-compatible microcomputer was used). 


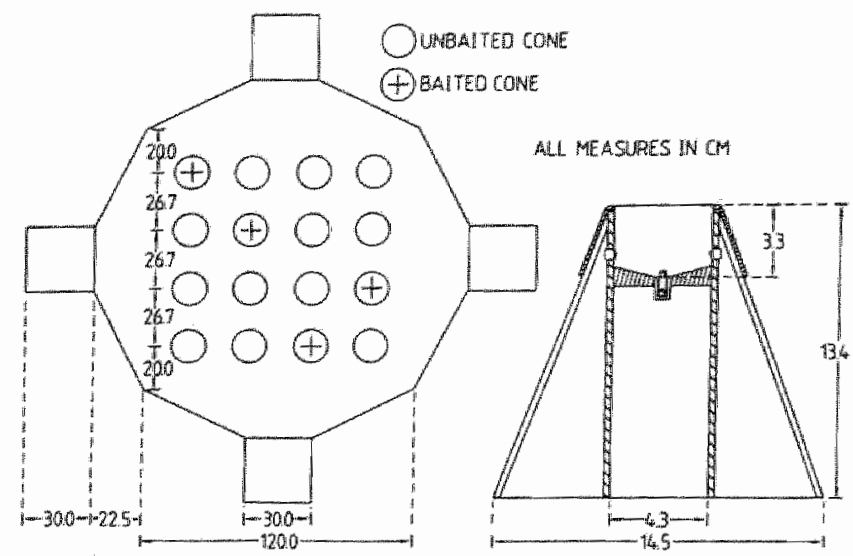

Fig. I. Map of the cone theld and constructional derails of a cone.

FGURE 1: Map of the cone-field and constructional details of a cone.

The experimental room was illuminated by four fluorescent red tubes and three 100-W bulbs which were adjusted by a dimmer to an intensity of 35-50 lux, measured on the floor of the apparatus. Extra-maze cues consisted of two doors, a one-way screen and a window (both covered by black curtains), a sink, a table with computer and interface, and a radial maze. The experimenter satin a chair in front of the apparatus. He was present and visible throughout all behavioral testing.

\section{Procedure}

One week after arrival at our laboratory, the weights of the animals were gradually reduced, within one week, to $85 \%$ of their free-feeding values. In the course of formal training, the young animals were allowed to gain weight $(5 \mathrm{~g} /$ week) in order to follow the growth curves of undeprived peers. The body weights of the senescent rats, on the other hand, were further reduced until they had reached $80 \%$ of their undeprived free feeding weights. This differential deprivation technique was applied in order to induce a comparable level of food deprivation between the age groups (cf. Goodrick, 1968; 1975).

All rats were familiarized with the cone-field in ten-minute sessions on four consecutive days. All cones contained one $45 \mathrm{mg}$ food pellet (Campden Instruments) during the adaptation sessions. Additional pellets were scattered on the floor of the apparatus.

The animals were trained with massed trials (days 1 and $2: 2$ trials/day; days 3 to 20:3 trials/day; day 21: 2 trials) to a total of 60 trials. Four cones of a fixed set contained one food pellet each (see Fig. 1). The configuration of baited cones was also used in its $90^{\circ}, 180^{\circ}$ and $270^{\circ}$ orientation. Two rats per age group were trained with each orientation of the baited cones (in the senescent group with one of the 
four orientations three rats were trained). The starting position within a series of daily trials was determined by random permutations of the numbers 1 to 4 . Each rat had its own random order of starting positions.

A trial started by placing a rat into a start box. The sliding door was opened immediately. As soon as the rat had entered the cone-field, the door was closed. A trial was terminated when a rat had found and consumed all four food pellets or when 10 minutes had elapsed, whichever event occurred first. The rat was then put back into its home cage. The cone-field was cleaned with a damp sponge, and the four cones were re-baited. The next trial was then started. Each trial with less than four cone visits was not counted and the rat was given an extra trial.

\section{Statistical analyses}

Three measures of training were subjected to statistical analyses: WM errors, RM errors, and choice correspondence of reinforced visits.

- WM errors were revisits to the cones of the baited set.

- RM errors were all visits and revisits to the never baited cones.

- Choice correspondence of reinforced visits was determined as follows: The sequences of reinforced choices of two subsequent trials were compared, and the longest common sequence was determined. This measure could range from 1 (completely different sequence) to 4 (identical sequence of all four reinforced visits). The measure reflects the variability of the pattern of cone visits, but it neglects all erroneous choices (van der Staay et al., 1986; 1989). Computer simulation revealed that (provided 4 cones are baited) the mean choice correspondence measure is 1.72 if the order of reinforced visits was purely random from trial to trial.

Means of blocks of 10 trialls each were calculated. Changes on the three variables in the course of formal training were assessed by a two factorial age by trialblock analysis of variance (ANOVA) with repeated measures on the last factor (Winer, 1971, p. 515 ff.). This analysis was supplemented by ANOVAs within age groups in the case of age by trialblock interactions. As we expected that the performance of the age groups was similar at the start of formal training, we compared the first block means by t-tests.

\section{Results}

One 33-month old rat hardly ever left the start box during the adaptation sessions and during formal training. The data of this animal were therefore excluded from further training.

Learning curves were analyzed over trialblocks 2 to 6 only, because of a fairly high incidence of incomplete trials within the first block of 10 trials. A Kruskal-Wallis analysis revealed that the young rats completed less trials than the aged animals (Chi ${ }^{2}$ approximation $=4.5, \mathrm{p}<0.05$ ), as shown by the number of pellets left (average \pm S.E.M.: young rats: $1.2 \pm 0.2$; senescent rats: $0.6 \pm 0.2$ ). From the second trialblock onward incomplete trials in both age groups occurred only incidentally, and young and senescent rats no longer differed. 
WM errors (see Fig. 2, left panel). On the first three trialblocks WM performance of the two age groups did not differ $(\operatorname{ts}(14)<2.1$, ns). The age groups showed a different change of WM errors over trialblocks (age by blocks interaction: $\mathbb{F}(4,56)=4.5, \mathrm{p}<0.01)$. The analyses within age groups confirmed that the number of errors decreased in the young rats $(\mathbb{F}(4,28)=7.7, p<0.01)$. The apparent increase in the number of errors in the senescent rats, on the other hand, was not confirmed statistically $(\mathrm{F}(4,28)<1$, ns).
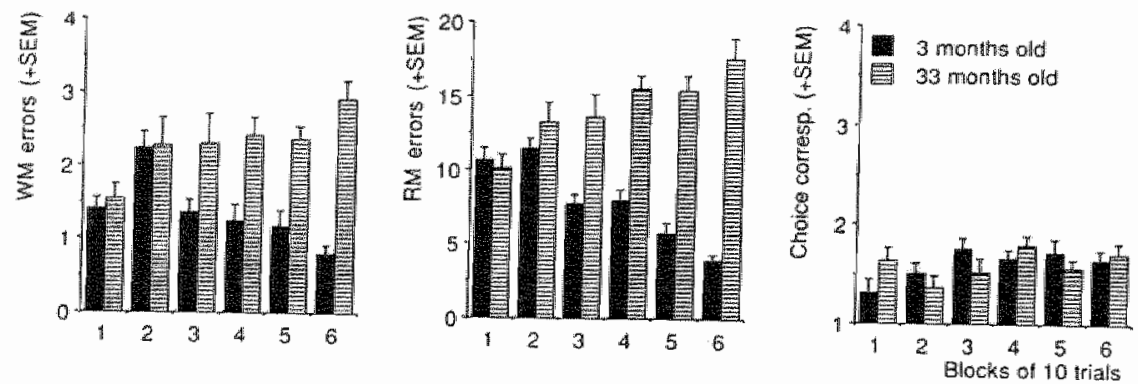

FIGURE 2: Mean number ( S.E.M.) of WM errors (left panel), RM errors (centre panel), and choice correspondence of rewarded cone visits (right panel) of 3.5- and 33-month-old female BN rats in a spatial discrimination task in a cone-field. Note that in trial block 1 the number of WM and RM errors are biased due to incomplete trials. Therefore, only trialblocks 2 to 6 were considered in statistical analyses.

RM errors (see Fig. 2, centre panel). On the first two trialblocks the age groups did not differ $(t s(14)<1.13$, ns). The two age groups differed on the development of RM errors in the course of training (age by blocks interaction: $F(4,56)=12.9$, $p<$ 0.01 ). The analyses within age groups confirmed that young rats learned to reduce their number of $\mathrm{RM}$ errors $(\mathrm{F}(4,28)=20.7, \mathrm{p}<0.01)$. The aged rats apparently increased their RM errors in the course of training. However, this impression was not confirmed statistically $(\mathrm{F}(4,28)=2.7, \mathrm{~ns})$.

Choice correspondence of reinforced visits (see Fig. 2, right panel). Choice correspondence always fluctuated around chance level and did not differ between
the ages on any of the trial blocks.

\section{Discussion}

An age-related decrease of spatial discrimination performance in the cone-field task was found. Both the WM and RM were impaired in the senescent rats. These results corroborate findings made in other 'free-choice' type mazes (e.g., Barnes, 
1979; Gage et al., 1984a; Ingram et al., 1981; van der Staay et al., 1986; 1988; Wallace et al., 1980).

There are no indications that the differences in WM and RM performance are due to differences between age groups on their initial levels of exploratory or locomotor activity. The senescent rats were as active as the young ones in all but the first adaptation session, as measured by the number of cone visits and the number of different cones visited (data not shown).

Motivational differences between ages were taken into account by applying differential deprivation techniques (Goodrick, 1968; Ingram et al., 1981); the older the rats are, the more their body weights are reduced in the course of training.

In order to disrupt the development of a fixed food-search pattern, the rats were randomly started from one of four start positions. Analysis of the choice correspondence of reinforced visits revealed that this procedure effectively prevented the rats from adopting a preferred sequence of visiting the baited cones.

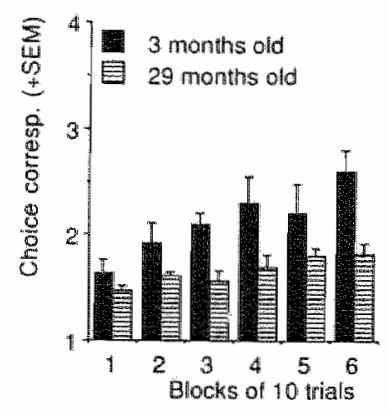

FICURE 3: Mean chotce correspondence of rewarded cone visits (+ S.E.M.) of three- and 29-monthwold male BN rats in a spatial discrimination task in a cone-field with one start position.

This finding contrasts with results from an experiment with an earlier version of the cone-field that was provided with one start position only. Five three-month-old and four 29-month-old male BN rats were used. The data were analyzed as described above. The age differences in WM and RM performance were comparable to those found in experiment 1. However, the choice correspondence showed a steady increase in the course of training (block effect: $F(5,35)=5.6, p<0.01)$, and the increase was stronger in the young than in the senescent group (age by blocks interaction $F(5,35)=2.5, p<0.05$ ) (see Fig. 3). This result illustrates that the interpretation in terms of WM and RM is problematic. It is not clear whether the better acquisition of the WM and the RM in the young rats was due to the development of a more efficient food-search pattern or because of superior place learning. 
Experiment II: The cone-field as a task for the longitudinal assessment of spatial discrimination learning

Most of the animal studies on age effects have been carried out with a cross-sectional design in which different age groups born at different times are compared in the same experiment. No distinction can be made between age and cohort differences in such a design. Unfortunately, there is a lack of longitudinal studies on complex learning and memory in animal research. An exception is the study by Bierley and co-workers (1986) who used a WM version of the radial maze.

The cone-field task might be valuable in longitudinal research on aging. Ideally, the learning and memory performances of the animals should not be affected by previous training on this task. All changes in the learning curves for WM and RM should be the result of developmental changes such as maturation or aging. The experiment described below was conducted to investigate whether the cone-field task could be used for the repeated acquisition of WM and RM in a longitudinal design.

\section{Materials and methods}

\section{Animals}

The involvement of the cholinergic input to the hippocampus was recently studied in rats that had received bilateral fimbria lesions, as has already been described in detail elsewhere (van der Staay et al., 1989). The untreated control rats $(n=8)$ of that experiment were used in the present study, and only the results of this group will be presented here.

Male Wistar rats (Bor:WISW(SPFCpb)), weighing approximately 280 grams, were supplied by Winkelmann, Versuchstierzucht GmbH \& Co. KG, Borchen, West Germany. They were housed individually in standard MakrolonTM cages with sawdust bedding in an air conditioned room (ca. $20^{\circ} \mathrm{C}$ ). The lights were on from $8: 00$ to $20: 00$.

\section{Apparatus}

The apparatus described in experiment I was used.

\section{Procedure}

The experimental protocol for food deprivation, the adaptation sessions and behavioral testing was similar to that used in experiment $\mathrm{I}$. The distribution of massed trials, however, was different from that in experiment I (day 1: 2 trials; days 2 and $3: 3$ trials/day; days 4 to $8: 4$ trials/day; days 9 to $15: 6$ trials/day; total: 70 trials). The rats were kept at $85 \%$ of their free-feeding weights during behavioral testing.

After the seventieth trial the rats were put on a reversed dark-light cycle (light on: $20.00 \mathrm{~h}$; light off: $8.00 \mathrm{~h}$ ) with food and water available ad libitum. Re-acquisition of spatial discrimination was assessed when the rats were 8- and 
12-month old. The procedures used in the re-acquisition sessions were identical to those of the original acquisition sessions. However, no adaptation sessions were given prior to re-acquisition. The rats were subjected to a total of 50 trials (days 1 to $3: 4$ trials/day; days 4 to $8: 6$ trials/day; day $9: 8$ trials), since the rats had reached asymptotic performance after about 40 trials during the original acquisition period (see Fig. 4).

\section{Statistical analyses}

Three measures of the training periods were analyzed: WM errors, RM errors and choice correspondence.

The means of blocks of 10 trials were calculated for the first 50 trials of the three training periods, comprising the original acquisition and the two re-acquisition periods. WM errors and $\mathbb{R M}$ errors were analyzed by a two-factorial (training period by trialblock) ANOVA with repeated measures on both factors.

\section{Results}

WM errors (see Fig. 4, left panel). The rats made, on average, the same number of WM errors (general mean: averaged over all trialblocks) in the different training periods $(F(2,14)=0.60$, ns). The rats reduced their number of WM errors over the trial blocks $(\mathrm{F}(4,28)=18.76, \mathrm{p}<0.01)$. Learning progress was similar in the three training periods $(\mathrm{F}(8,56)=1.00$, ns).
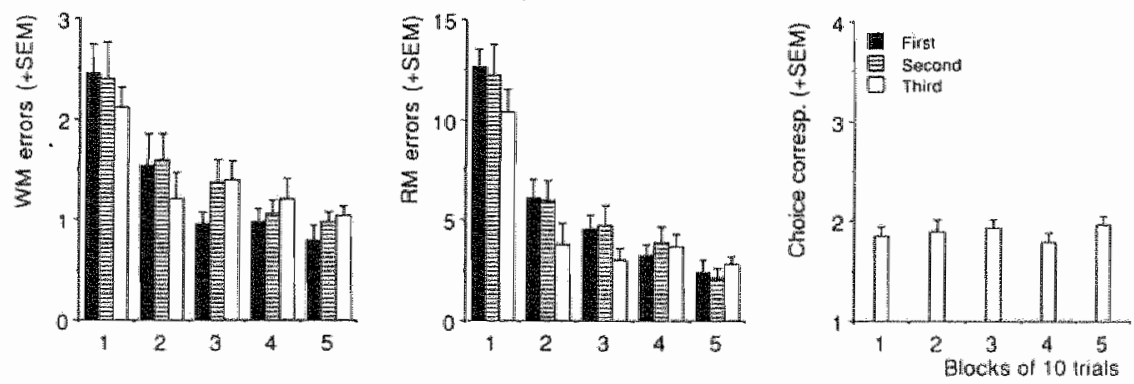

FIGURE 4: Mean number (+ S.E.M.) of WM errors (left panel) and RM errors (centre panel) of 8 malle Wistar rats in the first (acquisition), second and third (re-acquisition) tratining period. In the right panel choice correspondence of rewarded cone visits in the third training period is depicted.

RM errors (see Fig. 4, centre panel). There were no differences in the number of RM errors averaged over the trialblocks (general mean) in the three successive training periods $(\mathrm{F}(2,14)=1.72, \mathrm{~ns})$. The $\mathrm{RM}$ performance improved in the course of training $(\mathrm{F}(4,28)=131.77, \mathrm{p}<0.01)$, and the rate of improvement was similar in 
the acquisition and the re-acquisition periods $(\mathrm{F}(8,56)=1.99$, ns).

Choice correspondence of reinforced visits (see Fig. 4, right panel). Choice correspondence of reinforced visits was analyzed for the last re-acquisition period. The choice correspondence did not increase over the trialblocks $(F(4,28)<$ $1.00, \mathrm{~ns})$. It is conceivable that the development of a preferred food-search pattern might depend upon the position from which a rat starts. Therefore, an analysis on the choice correspondence per start position was performed for the second re-acquisition period. There was no difference in the choice correspondence between start positions (all $\mathrm{Fs}<1$, ns).

\section{Discussion}

The learning curves for WM and RM, obtained from the three successive training periods, had simllar shapes. Even after extended training, the rats had not developed a fixed pattern of visiting the baited cones. Retention of the previous training seemed to be absent. This lack of retention contrasts with the findings of Bierley and co-workers (1986), who observed that rats rapidly re-acquired critical performance in the radial arm maze task after an extended period without training. The absence of retention in the cone-field task with a four-month inter-test-interval is probably due to the complexity of the task. Thus, the cone-field task seems to be suitable for the repeated assessment of WM and RM performance in longitudinal studies.

\section{General discussion}

The aim of the present study was to validate the cone-field task as a complex place learning task that permits the simultaneous assessment of WM and RM.

We found that the WM and RM performance of old rats was clearly impaired, corroborating results from age-comparison studies with the holeboard (van der Staay et al., 1986; 1988). In the cone-field with one fixed start position, comparable age differences were found. In that version of the cone-field apparatus, however, the rats adopted a fixed food-search pattern in the course of training that was more pronounced in the young animals. Analysis of choice correspondence of reinforced visits revealed that randomization of the start position prevented the rats from developing a preferred food-search pattern. The second experiment explored the usefulness of the cone-field task for longitudinal research. Our results suggest that the learning curves are not distinguishable from those of naive animals if inter-test-intervals of at least four months are used. It is possible, however, that other inter-test-intervals are necessary for other strains of rats.

The validity of the cone-field to assess spatial discrimination is further supported by the finding that selective bilateral lesions of the fimbria disrupt WM and RM performance, confirming that intact hippocampal projections are important for the solving of the cone-field task (van der Staay, et al., 1989), as has been found with other complex spatial discrimination tasks (e.g., Jarrard et al,
1984 ). 


\section{Task requirements}

As early as 1929 Stone concluded that it is absolutely necessary for tasks designed for aging research that measures of cognitive performance are not confounded by factors such as speed of responding (cited by Goodrick, 1980). As WM and RM performance in the cone-field are entirely based on error scores, and as rats are allowed to complete a trial at their own speed, this requirement is met.

Olton (1988) points out that the testing procedure should make certain that the animals rely on the type of memory which the task is assumed to measure. This implies that the task must provide additional information, for example about the manner in which an animal solves the spatial discrimination (response strategies). In the holeboard, the predecessor of the cone-field, and in the conefield with one start position (see discussion, experiment I) animals developed a preferred food-search pattern in the course of training. The holeboard study involved one- and two year-old female rats of the inbred hooded CPBB strain (van der Staay et al., 1986). We found that it was mainly the younger rats that developed a preferred pattern of visiting the food containing locations.

Kobayashi and colleagues (1988) observed that young rats visited adjacent arms of a radial maze more frequently than aged rats, and that the frequency of visiting adjacent arms increased with training only in the young animals. This might imply that the young rats adopted a non-spatial strategy of 'running around in circles" (Yoerg \& Kamil, 1982). This strategy can facilitate the solution of a 'free choice" maze problem and may thus interfere with the interpretation of an improvement in spatial discrimination performance in terms of WM and RM processes (Olton, 1988). The cone-field task with four start position satisfies the requirement that the task is not solved by non-spatial strategies.

\section{Alternative measures}

The automatic registration of cone visits and of the exact time of every event makes it possible to analyze the rat's behavior in more detail. In addition to the spatial memory measures used in this study, other operational definitions can be used (compare Jarrard et al., 1984; Jucker et al., 1988).

If the trials are not completed within a predetermined time-limit, the error measures are likely to over-estimate the actual performance. If this occurs frequently, then the following alternative operational definitions of WM and RM provide less biased measures. (van der Staay et al., 1986; 1988). WM can be defined operationally by the ratio (number of food rewarded visits)/(number of visits and revisits to the baited set of cones). Thus this measure represents the percentage of all visits to the baited set of cones that had been reinforced. The alternative definition of RM is given by the ratio (number of visits and revisits to the baited set of cones)/(number of visits to all cones). This measure expresses the number of visits to the baited set of cones as a percentage of the total number of visits to all cones. Both measures have also been used by Devenport and Cater (1986) where they were labelled as 'efficiency' and 'accuracy', respectively.

Besides analysis of discrimination training, adaptation sessions which precede 
training in the cone-field can provide additional information. This comprises information about exploration and the speed of adaptation (increase of the number of cone visits over sessions), and whether scanning of the cone-field is exhaustive (number of different cones visited within a session).

\section{Applications}

With minor modifications the cone-field can be converted from a place learning into a cue learning task. The baited cones, for example, can be made visually distinct from the cones of the never baited set. In this manner it may be possible to investigate WM versus $R M$, and place versus cue learning in the same apparatus (e.g., Jarrard et al., 1984).

If one primarily is interested in the effects of experimental manipulations on search strategies, then the cone-field apparatus provides the option to use only one start position.

Ingram (1985) emphasized the potential value of longitudinal analyses in animal experimental models of aging. The result from our last experiment suggests that the cone-field can be used in this type of studies. The rats in this experiment showed no retention after a four months inter-test-interval and acquired the task as if they had had no previous experience in the apparatus.

Because the cone-field is a very complex task, rats will not perform faultlessly, even after extensive training. This opens the possibility to evaluate treatments which are assumed to improve spatial discrimination performance. Standard spatial discrimination tasks, such as the radial maze, may be less suited for this purpose because high levels of performance are often reached within a few (20-30) trials (e.g., Olton et al., 1977; Olton \& Samuelson, 1976). Investigations on this topic are currently performed in our laboratory.

In our experiments, BN rats up to 33 months of age were used. None of these animals showed motor impairments which would interfere with the leaning response against the cones. Thus, although aged rats have been found to show a decline in their locomotor abilities (e.g., Marshall, 1982; Wallace et al., 1980), the cone-field appears to be well suited for aging research, provided the rats are able to perform the required leaning response.

To summarize, the complex spatial discrimination task in the cone-field permits the simultaneous assessment of WM and RM. The tasks used in the present study appear to be suited for cross-sectional and longitudinal approaches in aging research. With minor modifications of the testing procedure or of the apparatus it is possible to address the issue of the development of a preferred pattern of visiting the cones and to investigate rat's behavior in non-spatial tasks. 


\title{
DIFFERENT TIME COURSE FOR AGE-RELATED CHANGES OF BEHAVIOIR IN A COMPLEX SPATIAL CONE-FIELD DISCRIMINATION TASK IN LEWIS RATS1
}

\author{
F. Josef van der Staay, Arjan Blokland and Wijnand Raaijmakers
}

\begin{abstract}
Clear age-related impairments in learning and memory performance have consistently been reported for rodents in spatial discirmination tasks. The aim of the present study was to evaluate whether Lewis rats show age-associated changes in spatial working (WM) and reference (RM) memory performance and whether the changes in their performance on both memory components follow the same time course. Rats of five different ages of the Lewis strain were trained on a complex spatial discrimination task in a cone-field. This task allows the simultaneous assessment of working memory and reference memory. We observed that WM performance declined already between 4 and 12 months of age. RM performance, on the other hand, was better in the 18-, 24-, and 30-month-old animals than in the two youngest age groups. This was probably because the younger rats adopted the habit of extensively inspecting non-reinforced places. Correlation analysis of the data supported the view that WM and RM represent different aspects of spatial memory. The study corroborates the notion that distinct aspects of behavior decline differently with age.
\end{abstract}

\section{Introduction}

Over the last decades a growing number of reports have focused on the cognitive changes that accompany normal aging. In rats, age-related deficiencies in learning and memory performance are especially prominent in tasks that require the use of spatial information (Rapp et al., 1987). When compared to the performance of young rats, senescent rats perform less well in complex spatial discrimination tasks such as the radial maze and circular platform (Barnes et al., 1980), the Morris water maze (Gage et al, 1984), and the holeboard (van der Staay et al., 1988; 1990d).

The distinction between working memory (WM) and reference memory (RM) has had a marked impact on theories about spatiall discrimination learning (Olton et al., 1979). When only a fixed subset of places in an apparatus contains reinforcements, and when a rat is free to visit all places in that apparatus, these two memory components can be assessed simultaneously. The WM holds information about the places that have been visited during a trial. The RM holds information about the places that are baited; this information is trial independent (Barnes, 1988).

Recently, we developed a cone-field (see van der Staay et al., 1990c) to study spatial discrimination learning. In the holeboard (Oades \& Isaacson, 1978), the 
predecessor of the cone-field, no clear criterial exist to distinguish between accidental and directed orientations towards the target (hole). This ambiguity might lead to different operational definitions of a hole visit, which in turn may influence the learning curves for the WM and RM, and hamper the comparison of results from various studies (cf. van Luijtelaar et al., 1989). In the cone-field, food-cups are placed in the tops of the cones. Every visit to the target (cone) is unambiguously operationally defined as a leaning response against the top of the cone (van der Staay et al., 1989). The visits are registered automatically. Clear age-related impairments of WM and RM performance have been observed in experiments comparing aged female (33-month-old) or aged male (29-month-old) Brown Norway rats with young (3-month-old) animals of the corresponding sex (van der Staay et al., 1990c).

The cone-field task has been used to investigate the effects of bilateral fimbria transection on spatial WM and RM in Wistar rats (van der Staay et al., 1989). The transection produces cholinergic denervation, predominantly at the more ventral part of the hippocampus. Fimbria-lesioned rats made about twice as many WM and RM errors as intact and sham-lesioned controls, even after extensive training. These results suggest that the cholinergic innervation of the ventral hippocampus plays a major role for the accurate performance in the cone-field task. This corroborates findings reported by others (summarized by Barnes, 1988) that post-lesion acquisition of place versions of spatial discrimination tasks is impaired. Interestingly, no such impairments have been reported for cue versions of these tasks (Barnes, 1988).

An experimental design including age groups covering the whole lifespan from adulthood to senescence is more adequate than an experiment with only two age groups when studying age-related behavioral changes (Coleman, 1989; Coleman et al., 1990; Ingram, 1985). We adopted such a multiple time point approach (Coleman et al., 1990) to compare the performance of 4-, 13-, 19-, and 30-month-old Brown-Norway rats in a spatial holeboard discrimination task (van der Staay et al., 1990d). A clear age-related decline in both WM and RM performance was found, an effect which was particularly pronounced between the ages of 19 and 25 months.

The present study was conducted to investigate, using a cone-field task, the age-related decline in spatial memory performance of Lewis rats of five different ages. We wondered whether the age differences observed in the holeboard study could be replicated in the cone-field. We expected that both WM and RM performance would decline in older animals, but that the decline would not necessarily occur during the same period of the lifespan. Distinctive aspects of behavior may show different time courses with respect to age-related changes. For example, Gage and co-workers (1988) have presented experimental evidence for the independent decline of different cognitive and non-cognitive abilities.

The complex spatial discrimination task in the cone-field allows the simultaneous assessment of different aspects of a rat"s behavior, e.g. the detection of a preferred food search pattern, and measures of spatial memory (discussed in: van der Stany et al., 1990 ). We therefore considered the cone-field task as 
especially suited to study whether the different aspects of these behaviors have different time courses. In addition, we determined the inter-dependencies of WM and RM performance by correlation analysis in order to test the notion put forward by Olton and Papas (1979) that the spatial working and reference memory are independent. This analysis makes use of the broad range of performance expected because of the broad range of age groups involved in the study.

\section{Materials and methods}

\section{Animals}

We used 45 male inbred Lewis rats of five different ages: 4-month-old ( $\mathrm{n}=10$ ), 12-month-old $(\mathrm{n}=8), 18$-month-old $(\mathrm{n}=10), 24$-month-old (range 22-25 months, $\mathrm{n}=8$ ), and 30-month-old (range 28-32 months, $\mathrm{n}=9$ ). All animals except the 4and 18-month-old rats were retired breeders. The rats were healthy during and after the experiment: no motor impairments or cataracts were observed.

As far as we know, there are no exact figures about the mean lifespan of Lewis rats. Lindsay and co-workers (1968) reported a survival of $26 \%$ at 24 months. We observed that the death rate of Lewis rats, which were kept in supply for experiments on aging in our animal house, started to increase from the age of 20 months, reaching a peak at about 28 months. Very few animals lived longer. The supplier of another line of Lewis rats (Charles River Wiga GmbH, West Germany) reports $50 \%$ survivability at $28-30$ months. Thus, animals from the two oldest groups might be considered as being senescent. The mean lifespan of Lewis rats is most likely shorter than that of Brown-Norway (30 to 32 months; Burek, 1978), a strain which we have used in an aging study on spatial holeboard discrimination (van der Staay et al., 1990).

All rats were housed individually in standard Makrolon TM cages on sawdust bedding in an air-conditioned room (ca. 20 $\mathrm{C}$ ). They were kept under a reversed light/dark cycle (lights on from 21:00 to 9:00).

\section{Apparatus}

The cone-field apparatus (see Fig. 1) has been described in detail elsewhere (van der Staay et al., 1989). The construction of the cones, however, was modified in order to improve the measures taken to prevent the localization of the baits in the cones by odor cues (see Fig. 1, lower right). All cones now contained a few inaccessible food pellets instead of just one. In addition, the height of the cones could now be adapted to the size of the rats, thereby making it necessary for rats to lean against the top of the cone in order to inspect the contents of a food cup (in the present study the height of the cones was adjusted to $16 \mathrm{~cm}$ ).

The experimental room was illuminated by four red fluorescent tubes and three 100-W bulbs, which were adjusted by a dimmer to give an intensity of about 50 lux on the floor of the apparatus. Extra-maze cues consisted of two doors, a one-way screen and a window (both covered by black curtains), a sink, a table with computer and interface, and a radial maze. The experimenter sat in front of the apparatus. He was present and visible throughout all behavioral testing. 

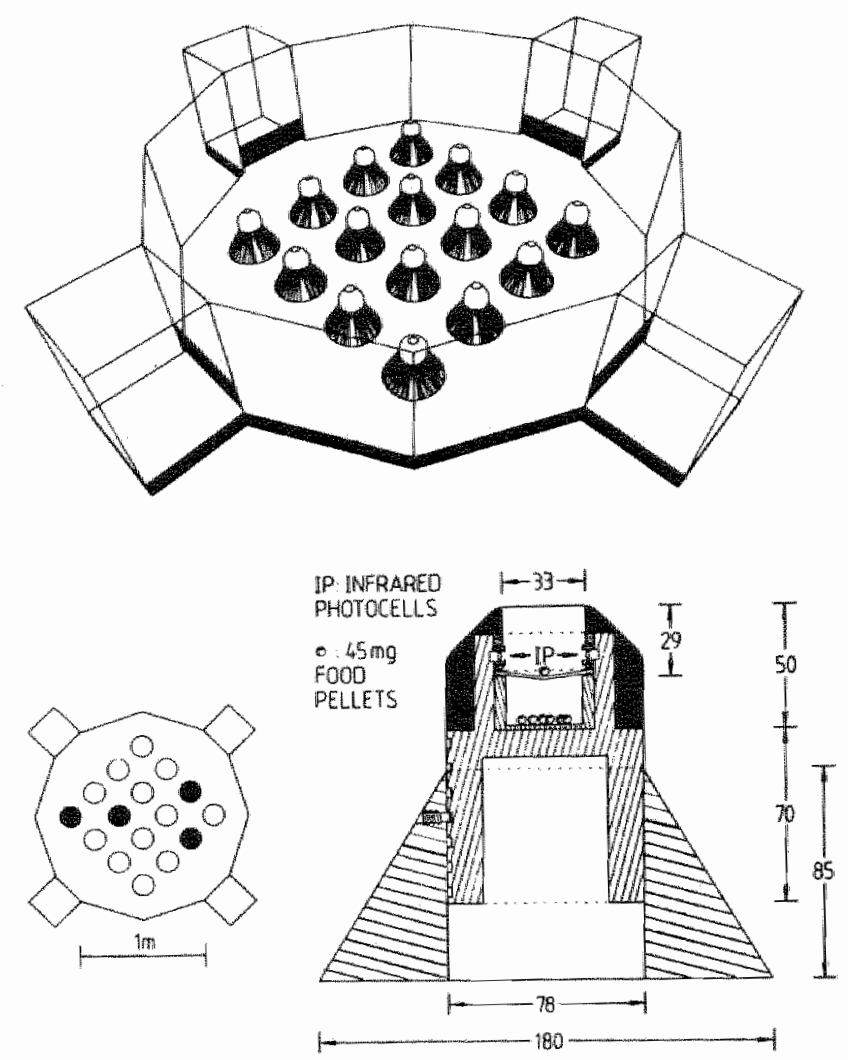

FIGURE 1: Upper panel: The cone-field apparatus. Lower left: Map of the cone-field; tilled circles. are baited cones, open circles are unbaited cones. Lower right: Cross-section through a cone in the cone-lield apparatus (measures are in mm). Cones were made of poly vinyl chloride, except the upper part (drawn black) which was made of brass. A cone visit was automatically registered whenever a rat touched the upper part of the cone. The entire top of a cone can be dismantled easily to refill the reservoir of pellets that provide masking odor cues. The height of the cone could be adapted (lowest position: 124: mm; highest position: $179 \mathrm{~mm}$ ).

\section{procedures}

There is a considerable increase in body weight with age in full-grown male Lewis rats: the 30 -month-old rats are approximately $40 \%$ heavier than the 
4-month-old rats. This increase in weight from adulthood (4 months) to senescence ( 30 months) fits a linear regression equation:

$$
\text { body weight (grams) }=8.1 \mathrm{x} \text { age (months) }+398
$$

A differential deprivation technique was applied in order to reduce motivational differences between age groups due to differences in body weight (Goodrick, 1968; 1980 ). Thus, the body weight of the rats was reduced to $85 \%$ (4-months), $80 \%$ (12and 18 -month-old), or $77.5 \%$ (24- and 30 -months) of their free-feeding values within one week. This deprivation technique produces a more equivalent relative body weight loss across the age groups.

Familiarization with the cone-field preceded formal training. The starting position was chosen randomlly for each of these adaptation sessions. All cones contained one 45-mg food pellet (Bioserve Dustless Precision Pellets) during the adaptation sessions, and additional pellets were scattered on the floor of the apparatus. Familiarization was terminated as soon as at least 15 pellets were collected from the food cups of the cones within one session.

All rats were then trained in massed trials (day 1:2 trials; days 2 and 3: 3 trials; day 4: 4 trials; days 5 to $13: 6$ trials) to a total of 60 trials. Within a series of daily trials, the starting positions were determined by random permutations of the numbers 1 to 4 . Each rat had its own order of starting positions.

Four cones of a fixed set contained one food pellet each. A trial was started by placing a rat in the start box. The sliding door was then opened immediately. As soon as the rat had entered the cone-field, the sliding door was closed. Whenever the rat touched the top of a cone, it closed an electrical circuit and activated a counter. This was scored automatically as a cone visit. Only contacts preceded by a visit to another cone were scored (exception: the first cone visit). Infrared photocells detected whether a rat poked its nose into a food cup. A MS-DOS compatible microcomputer collected the data and controlled the duration of the trials. A trial was terminated when the rat had found and consumed all four food pellets or when 7.5 minutes had elapsed, whichever event occurred first. The animall was put back into its home cage between trials. When the cone-field had been cleaned with a damp sponge, and the 4 cones had been re-baited, the next trial was started. All testing was done between 9:00 and 13:00 (which corresponds to the first phase of the dark period).

\section{Statistical analysis}

Five measures of formal training were subjected to statistical analysis:

- Working memory (WM): (number of reinforced wisits / number of visits and revisits to the baited set). This measure represent the percentage of visits to the baited set of cones that had been reinforced with a food pellet.

- Reference memory (RM): (total number of visits to the baited set / total number of visits). This measure represents the number of visits to the baited cones expressed as a percentage of all cone visits (baited and never baited).

- General working memory (GWM): (total number of cones visited / total 
number of visits). This measure represents the percentage of cone visits to the baited and never baited set that were visited at least once.

- Choice correspondence of reinforced visits: This measure reflects the variability of the pattern of visiting the baited set, but neglects all erroneous choices (cf. van der Staay et al., 1989).

- Mean inter-visit-interval (IVI) was determined by dividing the time between the first and the last visit in a trial by (number of visits - 1). This variable provides a measure for the speed of visiting the cones.

As there was a fairly high incidence of incomplete trials at the start of formal training, as indicated by the number of food rewards obtained (first trial block, mean number $\pm S E M$ of obtained food rewards: 4-month-old, $3.5 \pm 0.19$; 12 -month-old, $3.0 \pm 0.19 ; 18$-month-old, $3.5 \pm 0.08 ; 24$-month-old, $3.1 \pm 0.19$; 30 -month-old, $3.1 \pm 0.22$ ), the analyses were performed over trial blocks 2 to 6 only. From the second trial block onwards, the rats rarely failed to collect all food rewards. Even a low incidence of incomplete trials biases the error measures for WM, RM, and GWM. The ratio measures of WM, RM, and GWM are less biased by incomplete trials, and they are therefore presented here.

The means of blocks of ten trials each were calculated for all variables. Age differences in the overall level of performance (general mean) and in the shape of the learning curves were analyzed by a one-factorial ANOVA on orthogonal trend components calculated over the successive trial blocks (Winer, 1971). Where appropriate, the results of ANOVAs on differences between ages for particular trial blocks are included. Duncan post-hoc multiple range tests were performed in order to evaluate the age differences in more detail.

Product-moment correlation coefficients were calculated over all subjects for the measures WM and RM in order to evaluate their interrelationship. Again,
analyses were performed for trial blocks 2 to 6 only.

\section{Results}

\section{Working memory (WM)}

Averaged over all trial blocks (general mean) the WM performance was different for the age groups $(\mathrm{F}(4,41)=9.26, \mathrm{p}<0.01$; see Fig. 2A). Post-hoc analysis revealed that the 4 -month-old animals performed better than animals from the four older groups. All animals improved their WM performance in the course of training. This improvement was characterized by a general linear trend $(F(1,41)=$ $150.05, \mathrm{p}<0.01$ ) which explained $97 \%$ of the variation in the increase in WM performance. The age groups did not differ on the linear trend component $(\mathrm{F}(4,41)=1.40$, ns), indicating that the rate of improvement was similar for all
ages.

\section{Reference memory (RM)}

In the first phase of formal training no age differences were found (trial block 2: $F(4,41)=1.77$, ns; see Fig. $2 \mathrm{~B}$ ). The general mean was different for the different 
age groups $(\mathrm{F}(4,41)=14.10, \mathrm{p}<0.01)$. Post-hoc analysis of the general means
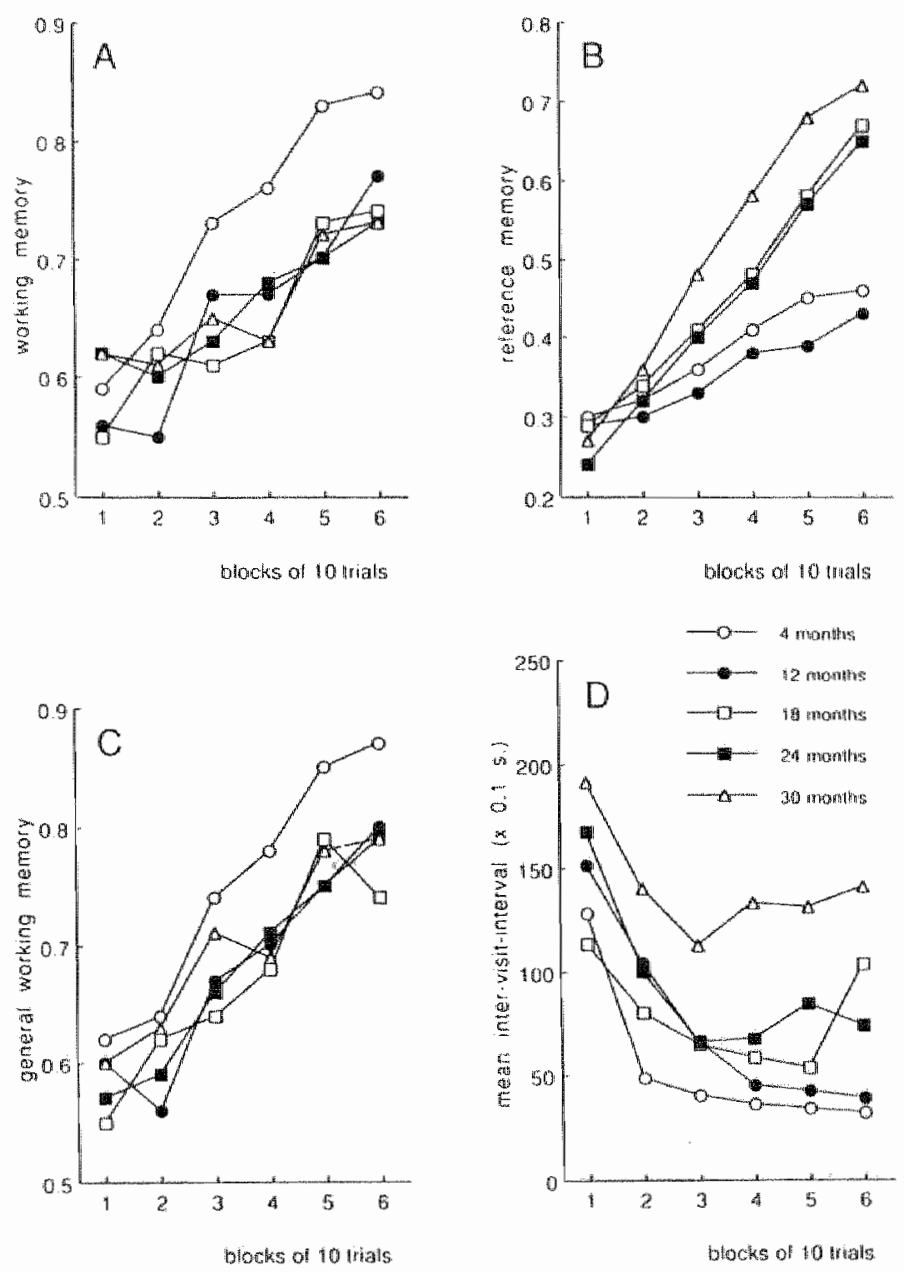

FIGURE 2: (A) working memory, (B) reference memory, (C) general working memory, and (D) inter-visil interval $(x 0.15$.) of Lewis rats of five different ages in a spatial discrimination task in a cone-field apparatus.

revealed that the 30-month-old rats performed best and that the 4 - and 12-month-old rats performed worst. The intermediate performance of the 18- 
and 24 -month-old animals differed from that of both the two youngest and oldest groups of rats. All age groups improved their performance in the course of training. This improvement was characterized by a general linear trend $(F(1,41)=$ $387.23, \mathrm{p}<0.01)$ which explained $99 \%$ of the variation. The age groups differed on the linear trend component $(F(4,41)=14.94, p<0.01)$. Post-hoc analysis of the linear trend components revealed that the RM performance of the 4- and 12-month-old animals improved slower than that of the three oldest groups.

\section{General working menory (GWM)}

The picture for GWM was very similar to that observed for WM. Averaged over trial blocks (general mean), the age groups showed different GWM performances $(F(4,41)=5.65, \mathrm{p}<0.01$; see Fig. $2 \mathrm{C})$. Post-hoc analysis confirmed that the performance of the 4-month-old animals was better than that the rats of the other four age groups. The increase in GWM performance during training was characterized by a linear trend $(\mathrm{F}(1,41)=349.09, \mathrm{p}<0.01)$ which predicted $97 \%$ of the variation. The age groups showed a parallel linear improvement in GWM performance $(\mathrm{F}(4,41)=1.39, \mathrm{~ns})$.

\section{Choice correspondence of reinforced visits (CC)}

There were no age differences in $\mathrm{CC}$ in any trial block. The $\mathrm{CC}$ fluctuated around the chance level. It is conceivable that the rats developed a fixed food search pattern per start position. However, the $C C$ still fluctuated around the chance level. when this measure was analyzed separately for the four start boxes.

\section{Mean inter-visit interval (IVI)}

When averaged over trial blocks 2 to 6 the IVI of age groups differed (general mean: $F(1,41)=6.53, p<0.01$; see Fig. $2 D)$. Post-hoc analysis revealed that the 30 -month-old rats visited the cones slower than the younger animals, the IVIs of which did not differ from each other. The IVI approached an asymptote rapidly, as indicated by the absence of general linear, or quadratic trend components $(\mathrm{F}(1,41)=0.58, \mathrm{~ns}, \mathrm{~F}(1,41)=0.96, \mathrm{~ns}$, respectively). It should be noted that trial
block 1 was not considered in the analysis.

\section{Correlation analysis}

The correlation analysis revealed that WM performance was not related to RM performance in any trial block (product-moment correlation coefficients for
blocks 2 to 6 , calculated over all animals $(n=45$ ) were $0.15,0.20,-0.04,-0.08$, and
-0.27 respectively; all associated probabilities: -0.27 respectively; all associated probabilities: $\mathrm{p}>0.10$ )

\section{Discussion}

The WM performance of the young rats was better than that of the older rats in the spatial cone-field task, as was expected; however, the RM performance of rats continued to visit the unbaited set of cones in the course of training more 
often than the older animals (poor RM performance), they made fewer revisits to the baited cones (better WM performance), and to the baited and never baited cones combined (better GWM performance). Neither the young nor the old rats developed a fixed pattern of visiting the baited cones. The correlation analysis supports the notion of Olton and Papas (1979) that WM and RM represent different aspects of spatial discrimination performance.

The better WM performance of the young rats corroborates results from holeboard studies (van der Staay et al., 1988; van der Staay et al., 1990d) and from earlier studies with the cone-field (van der Staay et al., 1990c). The decrease in WM performance occurred between 4 and 12 months in the present cone-field experiment. The finding that the RM performance of the 4- and 12-month-old rats was worse than that of the older rats contrasts with the normal pattern of age-related decreases in spatial discrimination learning (e.g. Barnes et al, 1980; Gallagher \& Pelleymounter, 1988b; van der Staay et al., 1990c).

Our study involved two age groups consisting of virgin rats $(4$, and 18 months) and three age groups consisting of retired breeders. The breeding history could act as an intervening variable affecting the results of comparisons between groups of rodents (cf. Ingram et al., 1983). We feel that it is not likely that the lack of breeding experience was responsible for the poor RM performance of the 4-month-old rats, because the performance of the 18-month-old rats was not affected. However, we cannot rule this possibility out.

Although the 4-month-old rats continued to visit the never baited cones, even after extensive training, they avoided revisits to both the baited and the never baited set of cones, as indicated by their better working memory performance (WM and GWM). Thus, the exploration of 4-month-old rats was very efficient. We assume that the 4 -and 12 -month-old rats have a tendency to investigate or explore the test environment more extensively than the older rats. It may be of ecological advantage to continue to inspect previously baited cones as they could potentially provide food (all cones had been baited during adaptation sessions). Although the 12-month-old rats had the same tendency to explore their environment as the 4-month-old animals, they made more revisits due to impairments of working memory (as indicated by both WM and GWM).

The poor RM performance of the 4-month-old rats did not affect their efficiency to obtain the food rewards when the time needed to collect the baits is considered. Despite the fact that they visited more cones, the 4-month-old rats obtained their rewards faster than the older rats (mean duration of trials of the last trial block in $\mathrm{s} \pm \mathrm{SEM}$ : 4-month-old, $42 \pm 3.6 ; 12$-month-old, $56 \pm 7.7$; 18-month-old, $52 \pm 7.4 ; 24$-month-old, $71 \pm 10.2 ; 30$-month-old, $114 \pm 9.0 ; \mathrm{F}(4,40)=$ $14.29, \mathrm{p}<0.011$.

An analysis of the number of different cones visited at least once during one trial provides an indication of the extent of exploration. All age groups visited approximately 12 different cones (including those of the baited set) in the second trial block $(\mathrm{F}(4,40)=1.95$, ns). The number of different cones visited decreased linearly with learning (linear trend: $F(1,40)=449.89, p<0.01$ ). The age groups differed with respect to this trend $(F(4,40)=9.98, p<0.01)$. Post hoc analysis 
confirmed that the decrease was more pronounced in the 18-, 24-, and 30 -month-old animals. In the last trial block, the mean number ( \pm SEM) of different cones visited by the 4-, 12-, 18-, 24-, and 30-month-old animals was, in order, $9.70( \pm 0.32), 10.6( \pm 0.43), 6.84( \pm 0.41), 7.48( \pm 0.52)$, and $6.56( \pm 0.26)$. The two youngest ages visited nearly the same number of cones as at the start of training; on average, they inspected about $60 \%$ of all cones during a trial.

None of the age groups developed a fixed pattern of visiting the baited cones, as the choice correspondence of reinforced visits fluctuated around the chance level. It appears that the young rats visited the cones in a rather arbitrary order, thereby relying heavily on their WM to avoid making revisits. They could have used scanning strategies, but these strategies were highly variable between and within subjects and were not detected by analysis of the data.

Olton $(1977 ; 1983)$ has extensively investigated whether rats use a strategy or algorithm to solve the radial maze task (e.g. through development of a fixed order of arm visits). He found that no such strategies were used; the order of arm-visits was too variable to depend on a particular rule or strategy. Olton concluded that rats relied on their WM. The choice behavior we observed for Lewis rats in the cone-field is comparable to that reported for rats in radial maze studies: a response strategy was not detected.

It could be argued that the cones were so close together that it would have been difficult to use spatial cues. The standard eight-arm radial maze has a central platform with a diameter of approximately $35 \mathrm{~cm}$ (e.g. Olton, \& Samuelson, 1976). The entrances of the arms are thus maximally $35 \mathrm{~cm}$ apart from each other. Rats are capable of discriminating with high accuracy between choice alternatives (arms) in such close proximity. In the cone-field, the minimum distance between choice alternatives (tops of the cones) is $26.7 \mathrm{~cm}$; the maximum distance is $113.3 \mathrm{~cm}$ (outer cones of diagonals) (cf. van der Staay et al., $1990 \mathrm{c}$, Fig. 1). The distance between choice alternatives (cones) thus appears to be
sufficiently large to allow the use of extra-maze the cone-field.

There is another reason why the cone-field task appears to be more complex than, for example, the radial maze. In the cone-field, an animal must learn to space. In contrast, the from unbaited cones, irrespective of its own position in centrall platform), whereas every in the radial maze is always the same (the choice point for the next cone visit (van der Stalth et al cone-field may serve as

Figure 2 shows that there were age-related differ. 1989). WM, GWM, RM and IVI. For the working medifferences between the measures animals performed better than the older animals. performance in the cone-field task could already. The decline in WM and GWM 12 months. On the other hand, the age differy be seen in Lewis rats at an age of between the age of $12-$ and 18 months. A third in RM performance occurred 30-month-old animals visited 18 months. A third measure, IVI, showed that the groups. This supports the notion more slowly than animals in the other age al., 1989) that the decline in performanc (e.g. Gage et al., 1988; Markowska et 
heterogeneous and that the aging process is not controlled by one common underlying mechanism.

Jucker and colleagues (1988) proposed that different pluysiological substrates underlie WM and RM. This assumption was based on their finding that, in an automated tunnel maze, the WM and RM performance of senescent rats (30 months) was impaired, whereas the WM but not the RM performance of middle-aged rats (17 months) was impaired, compared to the WM and RM performance of adult (5 months) animals. The authors considered that the results provided evidence for the hypothesis that the different physiological correlates underlying WM and RM are affected differently by aging. Following the line of reasoning of Jucker and colleagues (1988), one could argue that the better RM performance of the oldest rats in the present study was caused by an improved functioning of the underlying neural substrate. No experimental evidence, however, is available to support this view.

Lowy and co-workers (1985) used a modified T-maze procedure to investigate the age-related changes in WM and RM in rats. The RM component consisted of a spatial stem-discrimination. WM was assessed by discrete-trial alternation performance in two different mazes. The aged (22-24 months) rats showed an impairment in both WM and RM performance when compared with adult (8-9 months) animals. Clear neurochemical correlates of the age-related decrease in spatial discrimination performance were nor found.

To summarize, our data are in accordance with the idea that WM and RM represent different aspects of spatial discrimination performance. The notion that various aspects of behavior may change independently of each other during the lifespan is supported by the present experiment where the different behavioral measures were assessed simultaneously in the same animal and in the same testing environment. The present results are, however, probably limited to rats of the Lewis strain, since, in contrast to Lewis rats, both WM and RM performance in the cone-field task is better in adult BN rats than in senescent BN rats (van der Staay et al., 1990c). It is likely that our results on RM reflect an age-associated change in the prevailing habit or strategy used to solve the cone-field task rather than an improvement in the RM performance of the oldest animals.

The interpretation of measures for spatial WM and RM memory might be compromised by the development of extensive exploration tendencies. Thus, it is not appropriate to consider differences between young and old rats as an indication of a decline in spatial memory performance if age-related differences in the food-search habit occur. In that case, the age differences could (at least partly) be caused by the adoption of different behavioral strategies by the different age groups. This possibility should be kept in mind when studlying age related impairments of learning and memory.

In conclusion, attention should be paid to alternative explanations of the behavior operationally defined as WM and RM. Our results are consistent with Olton's (1988) notion that the heterogeneity of cognitive strategies and behavioral habits complicates the interpretation of age-related changes. 
Therefore, spatial discrimination tasks should allow a detailed analysis of rats' behavior in order to detect possible age-related changes in the strategies used to solve a task.

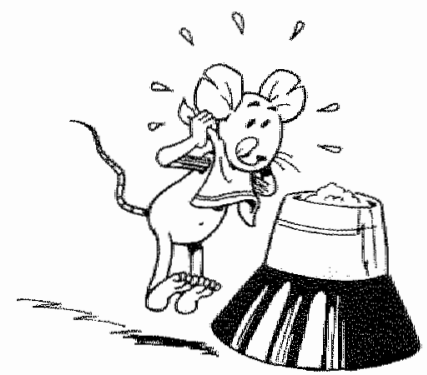




\title{
SPATIAL DISCRIMINATION LEARNING AND ORIENTATION STRATEGY IN YOUNG AND OLD LEWIS RATS1
}

\author{
Arjan Blokland, Wijnand Raaijmakers, Wiel Honig and F. Josef van der Staay
}

\begin{abstract}
In an earlier study we found that, in a conewfield task, the spatial reference memory performance of young Lewis rats was impaired compared to that of old rats. This obserwation did not corroborate findings from other studies reporting a consistent age-related decline in reference memory performance. It was suggested that the young Lewis rats adopted a habit of inspecting non-rewarded places. The present study was designed to evaluate the learning performance and the orientation strategy of young (4-month-old) and old (26-month-old) Lewis rats in a more simple cone-field task. During training, no differences between young and old rats were found in reference memory and working memory performance. Spatial discrimination ability was assessed during probe trials and evidence was found that both young and old rats used an allocentric orientation strategy.
\end{abstract}

\section{Introduction}

We developed a cone-field task to study spatial information processing in the rat and validated it as a task for the evaluation of age-related impairments in spatial discrimination learning (van der Staay et al., 1990c). The major advantages of the task are that different aspects of learning and memory can be assessed simultaneously and that the task controls for variables that could interfere with learning and memory performance such as the development of a fixed food search pattern, use of odor cues, differences in the speed of responding. Unexpectedly, we found that the performance of young Lewis rats was worse than that of middle-aged and old rats on the measure reference memory (RM) (van der Staay et al., 1990a). This finding does not corroborate our own results obtained with other strains of rats in the cone-field task (van der Staay et al ", $1990 \mathrm{c}$ ), and in the related hole-board task (wan der Staay et al., 1990d), and contrasts with the generally reported age-related decline in RM performance in other spatial discrimination tasks (e.g., Barnes et al., 1980; Rapp ett al., 1987). We suggested that the young Lewis rats might have adopted the habit of inspecting non-rewarded places, which would have interfered with RM performance.

The present study evaluated the performance of young and old Lewis rats in the cone-field apparatus. A modified procedure was used to investigate further the spatial ability of young and old Lewis rats. During the last phase of acquisition the rats were subjected to daily probe trials. This allowed us to assess the spatial bias of young and old rats, i.e. whether the rats had adopted an allocentric or egocentric orientation strategy.

1 This chapter has been published in Neuroscience Reserach Communications, 10 (1992) 105-11.0. 


\section{Materials and methods}

\section{Antmals}

We used 14 male inbred Lewis rats of two different ages, 4 months $(n=7)$ and 26 months (range, 2528 months; $n=7$ ). None of the rats had cataracts or motor impairments. All rats were able to lean against the cones. The animals were housed individually in standard Makrolon cages on sawdust bedding in an airconditioned room (about $20^{\circ} \mathrm{C}$ ). They were kept under a $12 / 12$ hour light dark cycle (lights on from 9:00 to 21:00).

\section{Apparatus}

The cone-field apparatus, which is fully automated, has been described in detall elsewhere (van der Staay et al, 1990a; 1990c). The experimental room was illuminated by four red fluorescent tubes and three $100-W$ bulbs, which were adjusted by a dimmer to give an intensity of about 50 lux on the floor of the apparatus. Extra-maze cues consisted of two doors, a one-way screen and a window (both covered by black curtains), a sink, and two tables, on one of which was a computer and interface. The experimenter sat in front of the apparatus. He was present and visible throughout the behavioral testing.

\section{Procedures}

We applied the same differential deprivation technique as in our previous study (van der Staay et al., 1990a) and reduced the body weight of the young rats to $85 \%$ and that of old rats to $77.5 \%$ of their free feeding values.

A few days after the start of food deprivation, the rats were placed in an adaptation box $(40 \times 74 \times 50 \mathrm{~cm})$ containing two cones, identical to those in the cone-field apparatus. Both cones contained two accessible $45 \mathrm{mg}$ food pellets (Bioserve Dustless Precision Pellets). A reservoir in the top of the cone was filled with four inaccessible food pellets. The rats had four 10-minute familiarization. sessions in the adaptation box. The box was in the same room as the cone-field apparatus but at a different location.

All rats were then trained in the cone-field with massed trials (days 1-1,0,2 trials; days $11-12,4$ trials; days $13-19,6$ trials), to a total of 70 trials. Three young and four old rats were started from start position 1 (see Fig. 1A) while the other rats were started from start position 2.

Four cones of a fixed set contained one food pellet each (see Fig. 1A). A trial was started by placing the rat in the startbox. The sliding door was then opened immediately. As soon as the rat had entered the cone-field, the sliding door was closed. Whenever the rat touched the top of a cone, it closed an electrical circuit and activated a counter. This was scored automatically as a cone visit. Only contacts preceded by a visit to another cone were scored (excepting the first cone visit). Infrared photocells detected whether a rat poked its nose into the top of the cone. An IBM-compatible microcomputer collected the data and controlled the duration of the trials. A trial was terminated when the rat had found and consumed all four food pellets or when 10 minutes had elapsed, whichever 
event occurred first. The animal was put back into its home cage between trials. After the cone-field had been cleaned with a damp sponge, and the four cones had been re-baited, the next trial was started. If a rat made less than four cone visits in a trial, which occurred only accasionally, the trial was skipped.

The rats were subjected to a probe trial during each of the last four acquistion sessions (days 16-19). The probe trial was the fourth trial of the series of six. The rats were started from the opposite start position of the cone field and no cones were baited. Probe trials were terminated after a rat had made five cone visits. All testing was carried out between 9:00 and 15:00 (which corresponds to the first phase of the light period).

\section{Statistical analysis}

Apart from the measures working memory (WM) (number of rewarded wisits / number of visits to the baited set of cones), reference memory (RM) (number of visits to the baited set of cones / number of visits to all cones), and choice correspondence of reinforced visits $(\mathrm{CC})$ (the longest common sequence of reinforced choices of two successive trials), which were also evaluated in our previous study (7), one additional measure was used to evaluate the performance during probe trials, namely reference memory errors (RMerrors). This measure represents the total number of visits to the non-baited set of cones.

Data from trial blocks 2-7 only were analyzed because of a fairly high incidence of incomplete trials (i.e. more than three cone wisits but not all reinforcements were collected) on the first block of 10 trials (rewards obtained in first trial block, mean number \pm SEM: young, $3.04 \pm 0.18$; old, $2.10 \pm 0.14$ ), which biases the RM and WM measures. From the second trial block onwards, rats only occasionally failed to collect all four food pellets.

The means of all variables were calculated for blocks of 10 trials, except for RMerrors, which were evaluated per probe trial. Age differences in overall performance (general mean) and in the shape of the learning curves were analyzed with a one-factorial ANOVA on orthogonal trend components calculated over the successive trial blocks (trials in the case of RMerrors) (Winer, 1971).

Product-moment correlation coefficients were calculated over all subjects between the measures WM and RM in order to evaluate their inter-relationship.

\section{Results}

\section{Reference memory}

The RM performance (Fig. 1B) of young and old rats was similar when averaged over trial blocks $2-7$ (general mean: $F(1,12)=2.03$, ns). The improvement in $R M$ performance $(F(1,12)=98.85, p<0.01)$ was characterized by a linear trend component $(F(1,12)=258.34, p<0.01)$ which explained $97 \%$ of the variation in the increase in RM performance over trial blocks. 

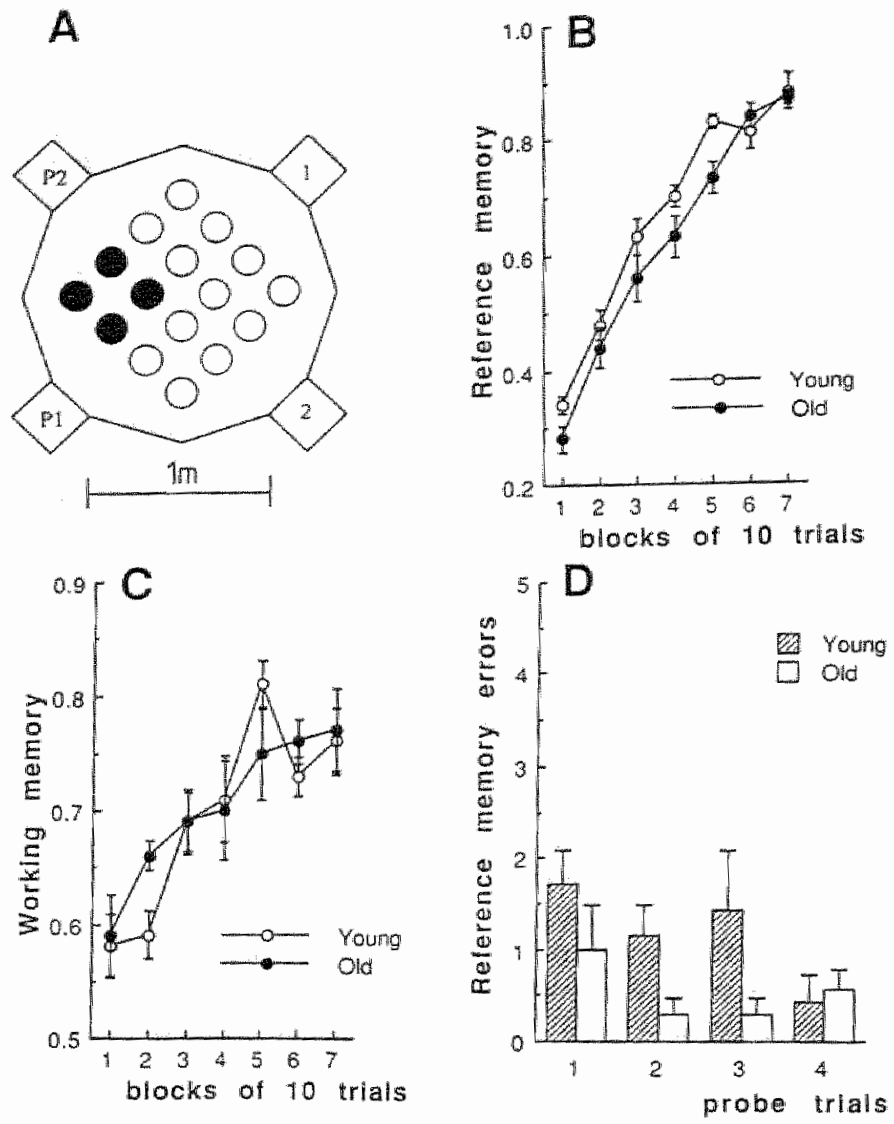

FIGURE 1: (A) Map of the cone-ficld apparatus; flled circles are baited cones, open circles are never baited cones. Numbers in start boxes refer to start positions during training (1 and 2), and probe trials (P1 and P2). The performance of Lewis rats of two ages in a spattal discrimination task in a cone-tield apparatus: (B) reference memory (number of visits and revisits to the baited set of cones / number of visits to all cones) $( \pm S E M)$, (C) working memory (number of rewarded visits / number of visits to the baited set of cones) ( $5 E M$ ), and (D) mean number ( + SEM) of reference memory ertors made in probe trials.

\section{Working menory}

Averaged over trial blocks 2-7, the WM performance (Fig. $1 \mathrm{C}$ ) of young and old rats did not differ (general mean: $F(1,12)=0.12$, ns). All animals improved their WM performance $(F(5,60)=8.19, p<0.01)$ in the course of training. This 
improvement was mainly characterized by a general linear trend $(F(1,12)=35,14$, $p<0.01$ ) which explained $76 \%$ of the variation in the increase in WM performance over trial blocks.

Choice correspondence of reinforced visits

There were no age differences for the measure choice correspondence of reinforced visits. Furthermore, the choice correspondence of both age groups did not deviate from the chance level (all ts $<0.94$, ns), indicating that both age groups visited the baited cones in a non-systematic order.

\section{Reference memory errors}

During probe trials old rats made fewer RMerrors (Fig. 1D) than young rats did (general mean: $\mathrm{F}(1,12)=6.89, \mathrm{p}<0.05)$. The number of RMerrors did not decreas over the successive probe trials $(\mathrm{F}(3,36)=1.85, \mathrm{n.s})$.

\section{Correlation analysis}

Correlation analysis revealed that there were no interdependencies for the measures RM and WM on any trial block. Calculated over all animals, the product-moment correlation coefficients for blocks $2-7$ were $0.04,0.41,0.29,0.28$, 0.39 , and 0.43 respectively (all associated probabilities: $\mathrm{ns}$ ).

\section{Discussion}

The RM performance of the young rats in the present study is similar to that of the old rats and both age groups eventually achieved an almost perfect performance level. This suggests that, in contrast to our previous study (7), the young rats did not develop a habit of inspecting unbaited cones in the present cone-field task. This is most likely caused by two procedural changes in the present study: firstly, no adaptation trials in which all cones were baited in the cone-field apparatus were given, and secondly, the baited cones were clustered in one corner of the apparatus.

Also, no age differences in WM performance were found in the present study. The WM performance of the young rats in the present study was lower than in our previous study (van der Staay et al., 1990a), whereas the WM performance of the old rats was similar to the performance in our previous study. The close proximity of the baited cones may have increased the susceptibility of the young rats to interference, resulting in an increase in revisits to baited cones. Old rats were not affected by this procedural modification.

The number of RMerrors during probe trials made by young and old rats was rather low. Furthermore, the RM performance during probe trails was comparable to the performance at the end of training in the cone-field task (approximately one RMerror to collect the four rewards).

It has been reported that rats can use different strategies, i.e. spatial-mapping, cue, and praxis, to solve a problem in the Morris water escape task (Whishaw \& 
Mitteman, 1986). It has been suggested that old 'learning-impared' rats use a non-allocentric orientation strategy in this task (Barnes et al., 1980; Blokland et al, submitted; Gallagher \& Peleymounter, 1988a). In the present experimert the rats could have used a praxis or ego-centric strategy because of the rather simple nature of the task (one start position and baited cones at a fixed position). Our finding, however, that the number of RMerrors was relatively low in young and old rats during probe trials suggests that the rats had adopted a spatial orientation strategy during acquisition. If the rats had adopted a praxis or an ego-centric strategy they would have visited the cones at the opposite side of the apparatus during probe trials and consequently would have made significantly more RMerrors during probe trials. Moreover, although visits to cones of the "baited" set were not reinforced, the rats made the most visits to these cones in probe trials. Unexpectedly, the old rats performed better than the young rats during probe trials, an observation for which we have no ready explanation. However, it must be noted that although there was statistical support for an age-related difference for RM errors, the absolute difference was rather small.

The test procedure used in the present study is comparable to the procedure used by Barmes et al. (1980) in which young and old rats were tested in an elevated T-maze: a fixed start and goal position. They reported that spatial discrimination learning was similar in young and old rats but that the old rats mainly used an egocentric orientation strategy whereas the young rats used an allocentric orientation strategy. Our results support the notion that no age-related spatial discrimination performance deficit is found in simple tasks (4). However, in contrast to Barnes et al. (1980) we found that old Lewis rats use an allocentric orientation strategy in the cone-field (this study, van der Staay et al, 1990a). A preserved ability to use an allocentric orientation strategy in old rats has also been reported for Long-Evans rats in the Morris spatial navigation task (Lindner \& Schallert, 1988). Taken together, these findings suggest that the ability to use an allocentric orientation strategy does not necessarily declines with aging.

Although the rats were started from one start position, there was no indication that the Lewis rats developed a fixed food search pattern. This does not corroborate the findings of earlier studies with Brown Norway rats (van der Staay et al., 1990c). When started from one start position with the baited cones in a fixed configuration, young but not old Brown Norway rats developed a fixed food search pattern (van der Staay al., 1990c). Because of the close proximity of the baited cones in the present study, it is feasible that the rats adopted a strategy to go to the region where the baited cones were located using spatial mapping, and once there, to rely on their WM. Such a strategy does not necessarily result in an increase in the measure $\mathrm{CC}$.

Correlation analysis revealed that WM and RM measured independent aspects of behavior, which corroborates the findings of earlier studies (van der Staay et al, $1990 \mathrm{c} ; 1990 \mathrm{~d})$. This indicates that, irrespective of procedural changes, the conefield task assesses RM and WM independently.

In summary, this study revealed that there is no age-related difference in RM and WM performance in Lewis rats in a more simple cone-field task. In probe 
trials, young and old Lewis rats showed a spatial bias towards the cones that were baited during training, indicating that they had used an allocentric orientation strategy during training. 



\title{
AGE-RELATED CHANGES IN SPATIAL DISCRIMINATION LEARNING PERFORMANCE IN LEWIS RATS
}

\author{
Arjan Blokland, Wiel Honig and Wijnand Raaijmakers
}

\begin{abstract}
The performance of Lewis rats of different ages was evaluated in the spatial Morris swim task. Compared with young and middle-aged rats, old rats showed an impaired acquisition of the standard task in experiment 1 . In the experiment 2 a learning set procedure was used to deternine the ability of the rats to acquire a learning set. It was found that all age groups were able to acquire a learning set from the first to the second acquisition training but not from the second to the third acquisition training. Furthermore, the results of experiment 2 indicate that old rats did not use distal spatial cues in the Morris task. In contrast to young rats, old rats did not benefit from a simpler procedure in the Morris task in which the start and platform position were fixed (experiment 3). In experiment 4 proximal cues were introduced. Old rats only used a cue when it coincided with the platform position. It was concluded that age-related performance deficits in learning tasks do not always reflect differences in learning and memory processes.
\end{abstract}

\section{Introduction}

In the last decade much attention has been given to animal models of the decline in learning and memory performance associated with aging. These animal models are valuable tools in the study of mechanisms involved in learning and memory. Moreover, possible treatments for the disturbances in learning and memory processes that accompany aging in humans could be evaluated in such models. A decline in learning and memory performance in aged rats has been found in short-term memory tasks (Wallace et al, 1980; Dunnett et al., 1988) and in spatial learning tasks (Barnes et al., 1980; Rapp et al., 1987). Consequently, tasks which measure either short-term memory processes or spatial learning are assumed to be valid animal models of the age-related decline in learning and memory (see Sarter, 1987; Gallagher \& Pelleymounter; 1988b; Olton, 1988).

Age-related performance deficits have been found in different tasks measuring spatial learning and memory in the rat (see Rapp et al., 1987). However, when comparing the performance of young and old subjects in learning and memory tasks, one should control for age-related differences in factors that could interfere with learning and memory performance, e.g., speed of responding, response strategies, and motivation (see Sarter, 1987; van der Staayet al., 1990c), as these factors could infuence the validity of the model used.

The Morris spatial navigation task has been found to reveal age-related performance deficits (e.g., Gage et al., 1984a) that are assumed to be due to an impairment in spatial information processing in old rats (Rapp et al., 1987). This suggests that the Morris task can be used as a model for the age-related decline in learning and memory. Studies which report impairments in the Morris task after 
hippocampal lesions support the notion that rats use spatial information in this task (Morris et al., 1990; Whishaw, 1987). Furthermore, it has been found that cholinergic antagonists impair spatial navigation (Whishaw, 1985). This indicates that the Morris task is sensitive to cholinergic dysfunction, which is, according to Lindner and Schallert (1988), a prerequisite for an animal model of aging.

However, the occurrence of an age-related performance deficit in the Morris task has been found not to be consistent over strains and experiments. For example, in contrast to Fischer rats, Lindner \& Schallert (1988) reported no agerelated deficits in spatial learning in the Morris task have in Long-Evans rats. This preserved spatial discrimination learning performance of old Long-Evans rats stands in contrasts with other studies reporting performance deficits in LongEvans rats (Aitken \& Meaney, 1989; Pelleymounter, Smith \& Gallagher, 1987; Rapp et al., 1987). Apart form the study of Aitken and Meaney (1989), old LongEvans rats eventually perform as well as young rats (Rapp et al., 1987; Lindner \& Schallert, 1988). However, old Sprague-Dawley and Fischer rats do not seem to reach performance similar to that of young rats (Gage, et al., 1984a; Lindner \& Schallert 1988). "Taken together, these findings suggest that there may be strain differences in spatial discrimination learning performance.

Although we have reported a consistent age-related decline in spatial discrimination learning in Brown-Norway rats, we found that, compared with young (4 and 12-month-old) rats, old (18,24 and 30-month-old) Lewis rats had a superior spatial discrimination performance in a spatial cone-field task (van der Staay et al, 1990a). This stands in contrast with the notion that spatial discrimination learning is impaired in old rats (see above). We assumed that the young Lewis rats adopted the habit of inspecting non-reinforced places.

In this study we evaluated the spatial discrimination performance of Lewis rats of different ages in the Morris swim task. The cone-field task can be regarded as more complex than the Morris task. Rats have to search for food in four different locations in the cone-field task whereas only one position has to be remembered in the Morris task. Furthermore, in the spatial cone-field task, young and old rats need extensive training ( 50 and 60 trials, respectively) to reach an asymptotic performance which is not errorless (about 7 visits to collect pellets from 4 baited cones). Apart from the difference in task complexity, the Morris swim task has a more aversive character than the cone-field task. This could prevent young Lewis rats from developing the habit of exploring the water tank, which could interfere with spatial discrimination performance. We hypothesized that old Lewis rats would perform as well as young rats in the Morris task.

In experiment 1 we tested rats of three different ages in the standard Morris task. In experiment 2 we introduced a learning set paradigm to evaluate whether rats of different ages are able to acquire a spatial learning set. A learning set is defined operationally as an increased rate of acquisition of a discrimination with novel stimuli as a result of previous experience with discriminations using a
similar experimental procedure but different discriminative stimuli (e.g., Zeldin
$\&$ Olton, 1986). In the present study a decrease in the mith \& Olton, 1986). In the present study a decrease in the mean escape latency on successive acquisition trainings was operationalized as a learning set formation. 
Furthermore, the learning set procedure offered the possibility to evaluate whether a change of environment affects the performance of the rats. If rats use distal spatial cues from the environment, the escape latency of the rats should increase during the first phase of acquisition in a new environment. In experiment 3 we tested young and old rats in a simpler task in which the start and platform positions were fixed. In the last experiment we evaluated the effects of two different proximal cues on the performance of young and old rats.

\section{Experiment 1: Acquisition of the standard Morris task in Lewis rats of three different ages}

\section{Materials and methods}

\section{Animals}

Male Lewis rats of three different ages (Young: 5-month-old, $n=8$; Middle-aged: 13-month-old, $n=8$; Old: 25-month-old (range 23-27), $n=7$ ) were used. None of the rats had cataracts. All rats were housed individually in standard Makrolon cages on sawdust bedding in an air-conditioned room (about $20^{\circ} \mathrm{C}$ ). They were kept under a 12/12 hour light dark cycle (lights on from 9:00 to 21:00).

\section{Behavioral procedures}

The rats were trained on the standard Morris spatial navigation task (Morris, 1981 ) in a black water tank with a diameter of $1.22 \mathrm{~m}$. The rats were started from four different, randomly chosen, start positions and trained to find an invisible platform (diameter $11 \mathrm{~cm}$ ) that was at a fixed position in the water tank, $1 \mathrm{~cm}$ below the surface of the water. The temperature of the water was $20-22^{\circ} \mathrm{C}$. A trial lasted until a rat had found the platform or until $60 \mathrm{~s}$ had elapsed. If a rat did not find the platform within $60 \mathrm{~s}$ it was placed on the platform for three seconds and then removed from the water tank. On the first day the rats were given 4 trials. The rats were given 8 trials per day on consecutive days. Training continued until the rats' performance had reached asymptotic level.

\section{Statistical analysis}

Differences in escape latency were analyzed per trial block of four trials on the first seven trial blocks. The performance of the rats during training was analyzed in a two factorial (age and trial blocks) analysis of wariance with repeated measures over triall blocks. In addition, differences in learning curves were analyzed by comparing the individual orthogonal trend components calculated over the first three trial blocks (Winer, 1971), which correspond to the first phase of acquisition. A Duncan-Waller post hoc analysis was performed on differences in overall performance (general mean) and on individual orthogonal trend components in order to specify the age-related changes in more detail. 


\section{Results and conclusions}

The latency to find the platform increased with age on trial blocks 1 to 7 (Fs > 3.78, $\mathrm{p}<0.05$; see Fig. 1). The overall decrease in escape latency during the first phase of acquisition (trial blocks 1 to 3 ) was generally characterized by a linear trend $(\mathrm{F}(1,20)=51.15, \mathrm{p}<0.01)$ which explained $95 \%$ of the variation. The age groups differed for the linear decrease in escape latency on the first three trial blocks (age $x$ block effect: $F(2,20)=9.69, p<0.01$ ). Post hoc analysis revealed that the linear decrease in escape latency was similar in the young and middle-aged rats but slower in the old rats. The difference in the linear decrease in the escape latency explained $88 \%$ of the variation of the age-related acquisition deficit.

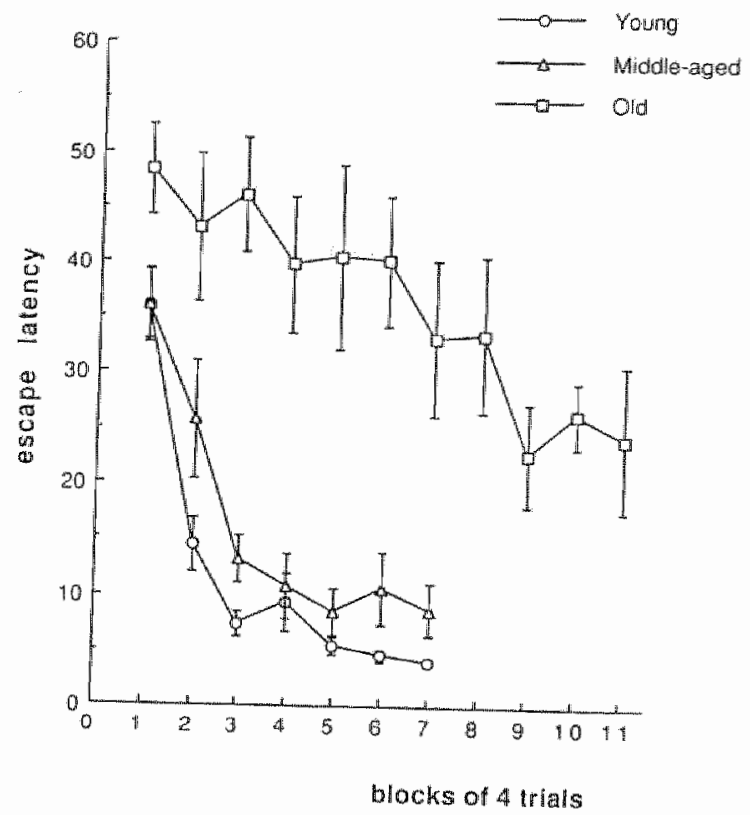

FIGURE 1: Mean escape latency per block of four trials (in seconds 1 SEM) of Lewis rats of different ages during acquisition of the Morris swim task with one platform position and four start positions.

Analysis of overall performance over seven trial blocks revealed that the performance was different for the age groups (general mean: $F(2,20)=33.79, p<$
0.01 ). Post hoc analysis showed that $\mathrm{p}$. 0.011 . Post hoc analysis showed that the performance of the old rats was worse
than that of the young and middle-aged rats; the performance of the young and middle-aged rats was similar.

The minimum mean escape latency per block of four trials of old rats was comparable to that of young rats in two blocks (see Table I). This finding argues against the explanation that the difference in escape latency is caused by an age-
related difference in swimming speed. Apparently, performance in the spatial 
Morris task is impaired in old Lewis rats. As this finding was not in accordance with our assumptions, the following experiments were designed to evaluate the nature of the spatial discrimination performance deficit of the old rats in the Morris task in more detail.

TABLE I: Minimum escape latency (in s) per block of four trials of individual rats for each age group in the Morris spatial navigation task.

\begin{tabular}{lccc}
\hline $\begin{array}{l}\text { Trial } \\
\text { block }\end{array}$ & Young & Middle-aged & Old \\
1) & 20.5 & 1.8 .0 & 32.0 \\
2) & 4.5 & 8.3 & 9.0 \\
$3)$ & 3.5 & 8.0 & 30.5 \\
$4)$ & 4.0 & 2.8 & 18.3 \\
$5)$ & 3.0 & 3.5 & 11.3 \\
$6)$ & 2.8 & 4.3 & 19.8 \\
$7)$ & 3.0 & 2.8 & 5.5 \\
$8)$ & - & - & 15.8 \\
$9)$ & - & - & 12.3 \\
10) & - & - & 18.8 \\
11) & - & - & 4.3 \\
\hline
\end{tabular}

Experiment 2: The performance of Lewis rats of three ages in a learning set paradigm

\section{Materials and methods}

Animals

The same rats as in experiment 1 were used.

\section{Behavioral procedures}

After the rats had reached an asymptotic level of performance in the standard Morris task (see experiment 1), the water tank was moved to another room. This room showed no similarities to the previous experimental room with respect to spatial cues. The rats were again trained on the same procedure as in experiment 1, two days (for the old rats) and three days (for the young and middleaged rats) after the first acquisition training. The rats were given four trials on day 1 and eight trials on day 2. After one day the water tank was again moved to another room. This room was somewhat similar to the second room, but instead of fluorescent light strips on the ceiling, two spot-lights $(60 \mathrm{~W})$ on the wall (spaced two meters) illuminated the room and provided distinct spatial cues.

Statistical analysis

The escape latencies were analyzed in a three-factorial (age, blocks and 
acquisition) analysis of variance. Repeated measures over acquisition were performed over acquisition training 1 to acquisition training 2, and acquisition training 2 to acquisition training 3 , separately. This was done to evaluate whether the mean escape latency of the rats decreased on successive acquisition trainings, which would imply that the rats had acquired a learning set. A Duncan-Waler post hoc analysis evaluated the age-related differences in more detail. In order to evaluate whether the performance of the rats was affected by changing the environment, we analyzed whether performance during the first block of acquisition training 2 and 3 differed from the asymptotic level of performance of acquisition training 1 (i.e. mean escape latency of last three blocks of 4 trials) by using t-statistics.

\section{Results and conclusions}

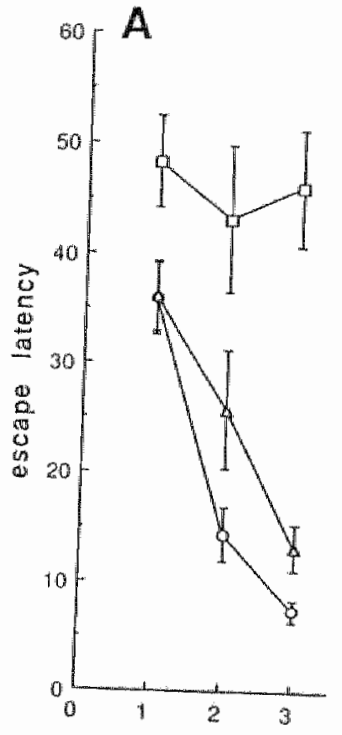

blocks of 4 trials

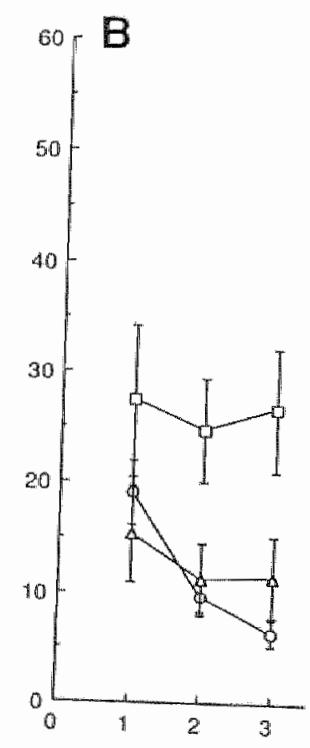

blocks of 4 tritils

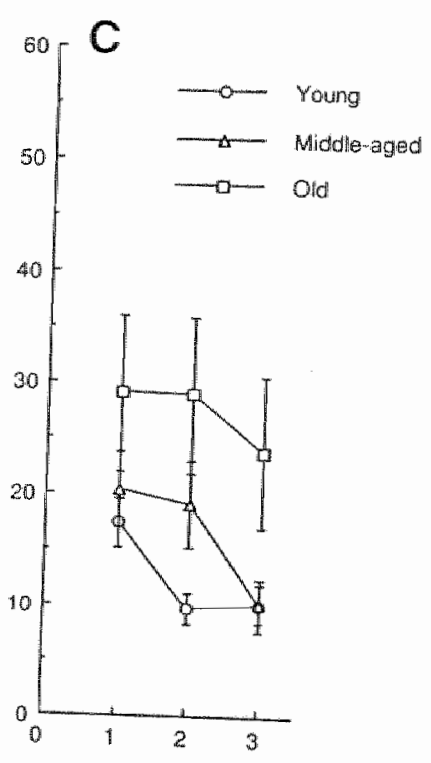

blocks of 4 irials

FIGURE 2: Mean escape latency per block of four trials (in seconds \pm SEM) of Lewris rats of different
ages during the first three trial blocks of the first ages during the first three trial blocks of the first (A), second (B), and third (C) acquisition training
of the Morris task with one platform position and four start positions.

The mean escape latency decreased during the first three blocks of the second acquisition training compared with the first three blocks of the first training (acquisition effect: $\mathrm{F}(1,20)=34.20, \mathrm{P}<0.01$; see Fig. $2 \mathrm{~A}$ and $2 \mathrm{~B}$ ), indicating that the rats had acquired a learning set. Although old rats performed worse than the 
age difference in the decrease in escape latencies from acquisition training 1 to 2 (age $x$ acquisition interaction effect: $F(2,20)=2.34$, ns). There was no decrease in escape latencies from acquisition training 2 to $3(F(1,20)=1.33$, ns; see Fig. $2 B$ and $2 \mathrm{C}$ ). In acquisition training 3 the old rats still had an impaired performance compared with young and middle-aged rats (general mean: $(F(2,20)=6.40, p<$ 0.01). Apparently, young, middle-aged and old rats were able to acquire a learning set from acquisition training $I$ to 2 but not on a subsequent acquisition training in the Morris task.

However, when we compared the performance during block 1 of the second acquisition training with asymptotic performance, we found that only the performance of the young rats differed $(t(7)=4.85, p<0.01)$. The performance of the middle aged and old rats during the first block of the second acquisition training did not differ from the asymptotic performance $(t(7)=1.39$, ns, and $t(6)=$ 0.48 , ns, respectively). Performance during block 1 of the third acquisition training differed from asymptotic performance for both the young $(t(7)=5.66, p<0.01)$ and middle-aged rats $(\mathrm{t}(7)=2.58, \mathrm{p}<0.05)$. The performance of the old rats during the first block of the third acquisition training was not different from the asymptotic performance $(t(6)=0.64, n s)$. This suggests that the performance of the old rats did not change after the water tank had been moved to other rooms whereas the performance of the young rats was affected during the first block on both occasions. The performance of the middle-aged rats was only affected when the water tank was moved to the third room.

Experiment 3: The performance of young and old Lewis rats in the water escape task with a fixed start and platform position.

\section{Materials and methods}

\section{Animals}

Young (4-month-old, $n=8$ ) and old (25-month-old, $n=8$ ) Lewis rats were used. None of the rats had cataracts. Housing conditions were identical to those in experiment 1.

\section{Behavioral procedures}

The rats were tested in the same water tank with the same procedures as in the previous experiments. However, the rats were now always started from only one start position and the platform position was fixed. Thus the rats could solve this task by using either a spatial or an egocentric strategy. The rats were trained until they had reached asymptotic levels of performance.

\section{Statistical analysis}

Escape latencies were evaluated over trial blocks in a two factorial (age and trial blocks) analysis of variance with repeated measures over trial blocks. In addition, differences in learning curves were analyzed by comparing the individual 
addition, differences in learning curves were analyzed by comparing the individual orthogonal trend components over the first three trial blocks, which correspond to the first phase of acquisition.

\section{Results and conclusions}

The old rats were found to have a greater escape latency during acquisition training than young rats $(F(1,14)=62.12, p<0.01$; see Fig. 3 ). During the first phase of learning (trial blocks 1 to 3 ), the rate of learning was different for young and old rats (age $x$ block interaction effect: $F(2,28)=13.79, p<0.01$ ). In contrast to experiment 1 , the age-related difference in the decrease in escape latency was now predominantly $(61 \%)$ explained by a difference in the quadratic trend component. At the end of training the escape latency of the old rats fluctuated around $20 \mathrm{~s}$ whereas the escape latency of the young rats was about $4 \mathrm{~s}$. The escape latency of young and old rats at the end of training (blocks 9 to 11) was comparable to the asymptotic level of performance of young and old rats in the allocentric task in experiment 1 . All old rats sometimes reached the platform within $5 \mathrm{~s}$, which argues against the difference being caused by a difference in swimming speed (see experiment 1 ).

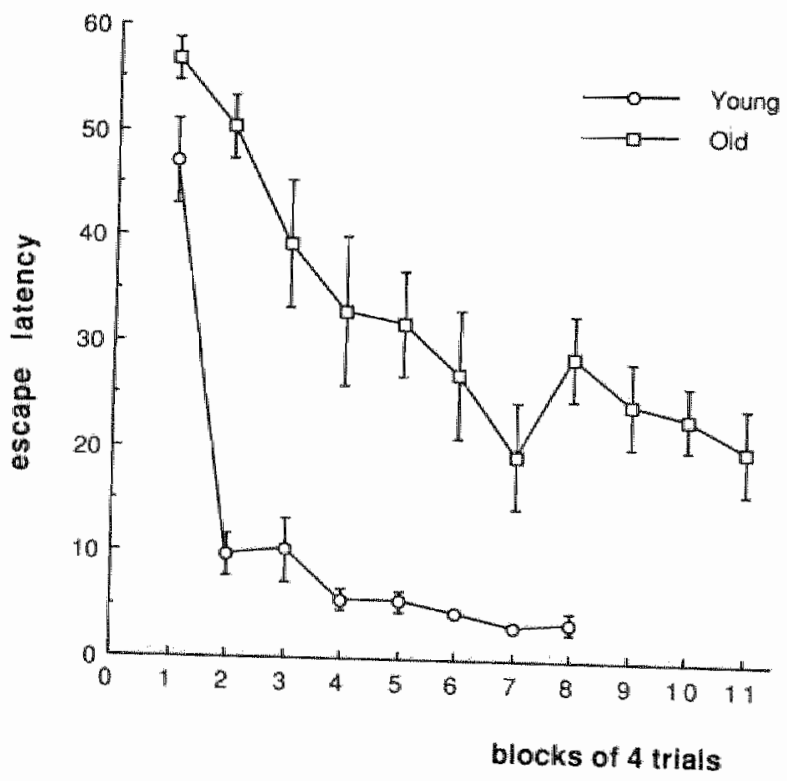

FIGURE 3: Mean escape latency per block of four trials (in seconds \pm SEM) of young and old Lewis rats
during acquisition of the Morris task with one platform poition during acquisition of the Morris task with one platform position and one start position. 
Experiment 4: The effects of introducing proximal cues to the testing environment in young and old rats.

\section{Materials and methods \\ Animals}

The same rats as in experiment 3 were used.

\section{Behavioral procedures}

After the rats had acquired the previous task (see experiment 3), the water tank was moved to another room (= room 2 in experiment 2) which was different from the room in which the previous task had been performed to prevent possible interference with spatial cues. In this experiment the rats were started from four different, randomly chosen, start positions. Per trial, the platform position was also moved to one of four different and randomly chosen positions in the water tank. The first cue was a yellow tennis ball hung $10 \mathrm{~cm}$ above the position of the platform, which was $1 \mathrm{~cm}$ below the surface of the water. After four blocks of four trials the water tank was again moved to another room ( $=$ room 3 in experiment 2) for the next task, again to rule out possible effects of interference of spatial cues. Instead of fluorescent light strips on the ceiling, two spot-lights $(60 \mathrm{~W})$ on the walls (spaced two meters) provided illumination. The procedures were the same as above but now the platform was covered with a white cotton cloth and was visible ( $1 \mathrm{~cm}$ above the surface of the water). Thus, in this task the tennis ball and the platform provided cues to the rats.

\section{Statistical analysis}

When the tennis ball was used as cue, escape latencies were ewaluated in a two factorial (age and block) analysis of variance over the four trial blocks. Differences between young and old rats in the task in which the tennis ball and the platform were used as cues were evaluated in a two factorial (age and trial block) analysis of variance over the first five trial blocks. In both analyses the data were analyzed with repeated measures over blocks. Age-related differences in the rate of learning were analyzed by comparing the individual orthogonal trend components callculated over successive trial blocks.

\section{Results and conclusions}

When the tennis ball acted as cue for the position of the platform, the old rats still had difficulty in locating the escape platform $(F(1,14)=52.03, P<0.01$; see Fig. 4A). However, the performance of the rats did not change over trial blocks (F(3,42) $=0.95$, ns). Young rats were able to locate the platform within 5 , suggesting that learning of this task procedure took place during the first trial block with no further improvement with additional training. The escape latencies of the old rats, however, fluctuated around 30 s, which suggests that they did not learn the procedure. When the platform itself was made visible the performance of the old rats was still poorer that that of young rats $(\mathrm{F}(1,14)=26.74, \mathrm{p}<0.01$; see Fig. $4 \mathrm{~B})$. 

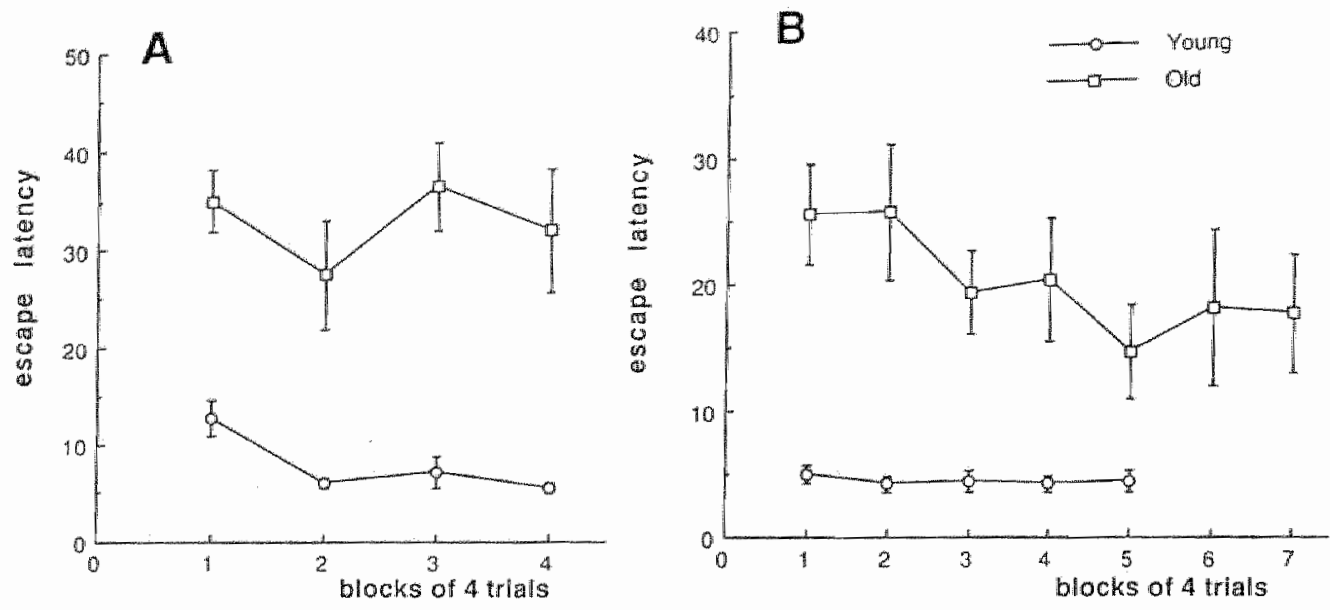

FIGURE 4: Mear escape latency per block of four trials (in seconds \pm SEM) of young and old Lewis rats during acquisition of the Morris task with, $(A)$ a tennis ball above the platform as cue, and (B) with platform as cue.

The performance of the rats improved over trial blocks $(\mathrm{F}(4,56)=3.09$, $\mathrm{p}<0.05)$. The improvement was for $85 \%$ characterized by a linear trend. There was weak statistical evidence (age $x$ linear trend interaction effect: $(F(1,14)=4.43,0.05<p<$ 0.10) that only the old rats improved their performance. The performance of the young rats was maximal from the first trial block onwards. The old rats were able to reduce their escape latency to less then $20 \mathrm{~s}$, suggesting that the combination of the tennis ball and the visible platform provided a better cue than the tennis ball alone. Data analysis showed that the mean escape latency during the last two trial blocks in the task with the tennis ball was greater than the mean escape latency in the task with the tennis ball and platform as cue $(t(7)=3.35$, $\mathrm{p}<0.05)$.

\section{General discussion}

In the first experiment old rats were slower in learning a spatial navigation task and did not reach a level of performance similar to that of young and middleaged rats. The possibility that this difference could be attributed to a slower swimming speed of the old rats could be ruled out. These findings corroborate the generally found impairment in spatial discrimination performance in old rats (Barnes et al., 1980; Rapp et al.r 1987; van der Staay et al., 1990c). However, the impairment in spatial discrimination learning of the old Lewis rats in the Morris task contrasts with our previous finding that old Lewis rats reach a fairly high level of spatial discrimination performance in the more complex spatial cone- 
field task (van der Staay et al., 1990a). Therefore, we assume that both tasks measure different aspects of spatial learning and memory. Moreover, spatial discrimination learning was compromised in the cone-field task because young rats reinspected non-rewarded places and in the Morris task because old rats did not use spatial cues (see below).

A possible explanation for this finding could be that different operationalizations of spatial discrimination performance were used which cannot be generalized over tests (cf. van Luijtelaar et al. 1989). In this study, place navigation in the Morris task was operationalized as the time taken to find the platform (swimming time is highly correlated with other measures of performance in the Morris task; Gage et al., 1984a) whereas place learning in the cone-field has been operationalized as visits to baited cones in relation to visits to non-baited cones (van der Staay et al., 1990a; 1990c). Another important difference between the Morris and the cone-field task is that the Morris task is aversively motivated (escaping from water) whereas the cone-field is not.

In experiment 2 we introduced a learning set procedure to evalluate the spatial ability of Lewis rats of three ages in the Morris task. Rats acquire spatial learning sets in a two-choice spatial discrimination task (Zeldin \& Olton, 1986). We found that the rats (young, middle-aged and old) reduced their mean escape latency from acquisition training 1 to acquisition training 2 but that the mean escape latency during acquisition training 3 was comparable to the escape latency of acquisition training 2 . This suggests that the rats had learned the task procedures but still had to learn to discriminate the spatial cues in the new environment, i.e., to form a spatiall map (O'Keefe \& Nadel, 1978). Efficient learning seems to take about 12 trials in the standard Morris spatial navigation task.

However, additional analysis revealed that the escape latency of old rats during block 1 of acquisition training 2 and 3 did not deviate from the asymptotic performance of acquisition training 1 . This suggests that changing the environment (i.e., changing spatial cues) did not affect the performance of old rats, which implies that old rats do not use spatial cues in the standard Morris task. In contrast to old rats, the escape latency of young rats during block 1 of the first and second acquisition training was increased compared with asymptotic performance whereas the escape latency of the middle-aged rats was increased only during the first block of the third acquisition training. Apparently, the performance of the young and the middle-aged rats was affected by changing the test environment, which indicates that they used spatial cues to acquire the task.

Because old rats did not use spatial cues to acquire the Morris task, we evaluated whether old rats were able to learn a simpler navigation task (experiment 3). It has been reported that old rats prefer to solve a task with a nonallocentric strategy (Barnes et al., 1980). Thus, old rats should show a good performance in the task with one start and one platform position. The difference in the decrease in escape latencies in the task with one start and one platform position was, however, predominantly explained $(61 \%)$ by a quadratic trend. In contrast, the age-related difference in the decrease in escape latencies in experiment 1 was predominantly explained $(88 \%)$ by a linear trend, indicating 
that the young rats did benefit relatively more from the test procedure in which the platform position and the start position were fixed during training. This finding corroborates our finding that, unlike old rats, young Brown-Norway rats develop a food search pattern in the cone-field task when they are started from one start position (van der Staay et al., 1990c). Furthermore, it was found that the asymptotic performance of the old rats fluctuated around $20 \mathrm{~s}$, which did not differ from the asymptotic performance found in the spatial task in experiment 1. This suggests that in the task with a fixed start and platform position, the old rats were not able to decrease the escape latency further than in the allocentric task, indicating that irrespective of the testing procedures, the old rats used a similar strategy in both tasks. This could be explained by the notion that old rats have difficulty in shifting strategies (see Lindner \& Schallert, 1988). Irrespective of the training procedure, the rats learn to associate the platform with escaping from the water during the first trials in the swimming pool. During the first training trials it was observed that the rats swum in an arbitrary manner. If old rats have difficulties in shifting from one strategy to another, they will continue to use the strategy used in the first trials in both procedures, i.e., swimming in an arbitrary manner. Such a strategy may be similar to the non-allocentric strategy observed in rats after hippocampectomy (Morris et al, 1990) or after administration of cholinergic drugs (Whishaw, 1985).

In experiment 4 we introduced a proximal cue (a tennis ball just above the platform). The relative high escape latency (30 s) of the old rats indicated that the old rats were not able to use this cue whereas the young rats were. When the platform was made visible, the old rats tended to improve their performance and the mean escape latency was somewhat shorter than $20 \mathrm{~s}$. The young rats did not show any improvement but performed well from the first trial block with no further improvement. This suggests that if the cue and the platform coincide, old rats are able to use this cue in the Morris task, as has also been reported in other studies (e.g., Rapp et al, 1987). However, in our study old rats did not reach a performance level comparable to that of young rats. This discrepancy is probably due to a different experience with test procedures. In our study the rats were first trained on the task with a fixed start and platform position and then trained on the cue task. As mentioned above, it could be argued that the old rats did not shift their strategy in the cue tasks and therefore did not use the cue optimally.

In studies to examine age-related changes in performance in the Morris task, several procedures have been developed to determine the nature of the agerelated decline in spatial discrimination performance. One way in which this has been assessed is by first training the rats to a steady performance and then removing the platform on a subsequent trial (e.g. Pelleymounter et al., 1987) or moving the platform to another quadrant of the water tank (e.g. Gage et al., 1984a). The spatial bilas of the rats can be assessed by two measures: duration of time spent per quadrant and annulus crossings (i.e., number of crossings of the previous platform position). However, it is unknown whether young and old rats deal differently with a situation in which the platform cannot be found or can be found at a different location. Moreover, if old rats are more rigid in their 
behavior (Goodrick, 1975; Sarter \& Markowitsch, 1983), the interpretation of these data is complicated. In a learning set procedure the use of an allocentric orientation strategy can be evaluated without these problems. Our data clearly show that the old Lewis rats did not use an allocentric strategy. Such a conclusion cannot be drawn from the procedures mentioned above, although the old subjects in those studies may also have used a non-spatial strategy. Pelleymounter et al. (1987) reported that, compared with young rats, old rats are impaired when the configuration of stimuli has been made salient. Although these data were interpreted as evidence that age-related differences in performance in the Morris task cannot be attributed to perceptual deficits, they could also be interpreted as evidence that old rats do not use a spatial orientation strategy. This latter interpretation would be in agreement with the findings of our study. In addition, our findings suggest that old Lewis rats can only use a salient proximal cue if it coincides with the platform (see above).

In conclusion, this study showed that old Lewis rats do not use a spatial orientation strategy in the Morris task but use a non-allocentric strategy to solve the task. Apparently a similar strategy was used in a task with one start and one platform position. The performance of the old rats was interpreted in terms of a difficulty to shift strategies. Furthermore, it was found that old rats can use a cue only when it coincides with the platform position. The present findings do not corroborate an earlier study with old Lewis rats in which it was found that old rats used a spatial orientation strategy (Blokland et al., 1992; van der Staay et al., 1990a). This may be due to different operationalizations of place learning and the difference in aversiveness of both tasks. The present results suggest that the use of a spatial orientation strategy is task dependent in young and old Lewis rats. If old rats do not use a spatial orientation strategy in the Morris task, age-related performance deficits cannot be explained in terms of an impairment in spatial discrimination learning. The finding that only minor age-related impairments in the Morris task are found in Long-Evans rats (Lindner \& Schallert, 1988) could indicate that old Long-Evans rats use a spatial orientation strategy and, therefore, perform as well as young rats. Taken together, these findings suggest that before interpreting age-related performance deficits in terms of learning and memory impairments, it has first to be established whether young and old rats make use of the same strategy to solve a task. In general, we think that studies which evaluate age-related differences in learning and memory should also evaluate different aspects of behavior that may affect the learning and memory performance, preferably in different tasks. This may reveal more information with respect to the processes underlying cognitive aging.

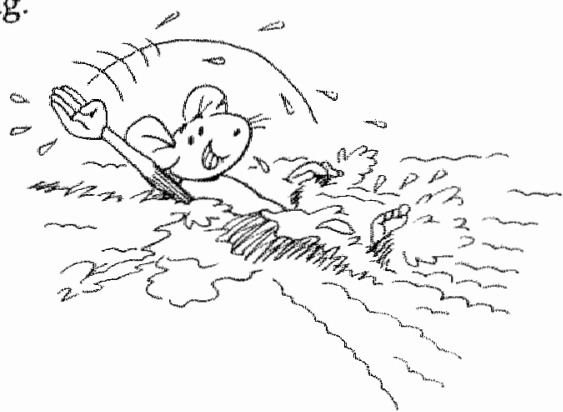





\title{
AGE-RELATED CHANGES IN THE RESPONSE TO STRESSFUL STIMULI IN DIFFERENT RAT STRAINS
}

\author{
Arjan Blokland, Wijnand Raaijmakers and F. Josef van der Staay
}

\begin{abstract}
Open-field behavior and two-way active avoidance learning in a shuttle box (SB) were assessed in adult, middle-aged, and old Brown-Norway (BN) and Lewis (LEW) rats. Performance in both tasks was found to be strain dependent. Aging affected open-field behavior and acquisition of the SB task differently in the rat strains. Both strains revealed atypical performance in the SB task: no age differences were found in BN rats whereas old LEW rats were found to learn the SB task faster than adult LEW rats. In an additional group of young and old Wistar rats the generally reported age-related decline in leaming performance was fownd: young rats performed better than old Wistar rats and showed an almost perfect performance at the end of training. We hypothesized that the difference in performance of the SB task depends on strain- and aggerelated differences in reactivity to stressful stimuli. The results suggest that agerelated differences in learning tasks with an aversive component may be confounded by an age-related change in the response to stressful stimuli.
\end{abstract}

\section{Introduction}

Learning and memory deficits have been found in different animal models of aging (Dunnett et al., 1988; Gallagher \& Pelleymounter, 1988b; Goodrick, 1972). Age-related impairments in performance in cognitive tasks, however, should be interpreted with caution because of possible age-related changes in, for example, response strategies or non-cognitive aspects of behavior such as motivation or speed of responding (Sarter, 1987; van der Staay et al., 1990C). It has been reported that rat strains differ in their physiological and behavioral response to stressors (Gilad \& Shiller, 1989; McCarty \& Kopin, 1978). Because there is an age-related change in the physiological reaction to stress (Sapolsky et al., 1983), it could be argued that the behavioral response to stress is also different in aged rats. Agerelated differences in rats have been reported in aversively motivated learning and memory tasks (e.g. shock (Ingram, 1988; Sprangler et al., 1989) and water (Gage et al, 1984a; Gallagher \& Pelleymounter, 1988b)). It is conceivable that these differences are, at least partially, caused by age-rellated differences in the unlearned behaviorall response to a stressor. Although Ray and Barret (1973) considered this possibility already in 1973 , no studies have evaluated this problem to our knowledge. We therefore assessed the effects of aging on open-field (OF)

behavior and two-way active avoidance learning in a shuttle box (SB) using rats of different ages and different strains. Increased responsiveness to the aversive characteristics of the OF should express itself in a decrease in the number of line crossings and an increase in the time spent in the corner squares (van der Staay et al., 1990b). An attempt was made to relate learning performance in the SB to the 
behavioral reactivity to a stressful stimulus. If aging affects the learning performance in the SB this should be comparable for different rat strains.

\section{Materials and methods}

\section{Animals}

In an open-field (OF) experiment, we used male Brown-Norway (BN) and Lewis (LEW) rats of three different ages (BN: 8 months, $\mathrm{n}=8 ; 14$ months, $\mathrm{n}=8 ; 26$ months, $\mathrm{n}=8$; LEW: 6 months, $\mathrm{n}=8 ; 13$ months, $\mathrm{n}=8 ; 25$ months, $\mathrm{n}=7$ ). In a second experiment the acquisition of a $\mathrm{SB}$ task was assessed. In addition to the $\mathrm{BN}$ and LEW rats, male Wistar (WIS) rats of two ages (6 months, $\mathrm{n}=5 ; 22$ months, $\mathrm{n}$ =6) were tested to ensure that rats were able to acquire the $\mathrm{SB}$ task in the present study. All rats were housed individually in standard MakrolonTM cages on sawdust bedding in an air-conditioned room (about $20^{\circ} \mathrm{C}$ ). They were kept under a 12/12 hour light-dark cycle (lights on from 9:00 to 21:00).

\section{Procedures}

OF behavior was assessed in a square base $(100 \times 100 \mathrm{~cm})$ subdivided into 36 equal squares by black lines. Two red fluorescent strip lights provided very $\mathrm{dim}$ illumination (about 2 lux) on the floor of the apparatus. Immediately after a rat was placed in the center of the OF, its movements were scored. The total number of line crossings and the total time spent in the corner squares were recorded manually with an MS-DOS compatible personal computer. These measusers were taken as indices of emotional reactivity (van der Staay et al, 1990b). Testing was carried out on four consecutive days in daily five-minute sessions.

Acquisition of shock avoidance learning was assessed in an automated SB. The apparatus consisted of two equal compartments $(21 \times 13.5 \times 20 \mathrm{~cm})$ separated by a barrier (height $3 \mathrm{~cm}$ ). The floor consisted of a metal grid connected to a shock scrambler. The animals were trained in one session of 50 trials. The session was preceded by an adaptation period of $5 \mathrm{~min}$. A trial started with the presentation of an auditory stimulus as a warning signal. The signal was terminated when a rat crossed the barrier within $8 \mathrm{~s}$; otherwise, a footshock $0.25 \mathrm{~mA}$ for young, 0.275 $\mathrm{mA}$ for middle-aged, and $0.30 \mathrm{~mA}$ for old rats) was given. The footshock was terminated when the rat escaped to the other compartment or when $10 \mathrm{~s}$ had elapsed. The shock intensity was gradually increased with age because of the agerelated decrease in shock sensitivity (unpublished datal). The inter-trial interval (ITI) varied at random between 35 and $60 \mathrm{~s}$.

The data of the OF test were aggregated over days to enhance reliability

1 Shock sensitivity was assessed behaviourally (see Cahill \& McGaugh, 1990) by finch reactions. Although we were able to assess the shock sensilivity of the Lewis and Wistar rats, the Brown-Norway rats did not show a flinch reaction to shock. We were therefore unable to determine whether there was an age-related decrease in shock sensitivity in Brown-Norway rats. It was assumed that the age-related decrease in shock sensitivity in the Brown-Noway strain was
similar to that of the Lewis and Wistar strain. 
(Ossenkop \& Mazmanian, 1985). The mean time (in tenths of seconds) spent in the corner squares was transformed to the natural logarithm ( $\mathrm{Ln}\left[\left(\mathrm{t}_{1}+\mathrm{t}_{2}+\mathrm{t}_{3}+\right.\right.$ $\left.\mathrm{t}_{4}\right) / 4$ ]; subscripts refer to day 1 through 4 of testing) in order to remove inhomogeneity. The data of the OF test were analysed in a two-factorial (age by strain) analysis of variance. In the SB task the overall level of performance (mean number of avoidance responses per block of 10 trials) was assessed. The data of the SB task were analysed in a three-factorial (age by strain by trial block) analysis of variance with repeated measures over trial block. Age differences in overall performance (general mean) and in the shape of the learning curves within strains were analysed with a one-factorial ANOVA on orthogonal trend components calculated over the successive trial blocks (Winer, 1971). Correlations between measures were evaluated using Pearson's correlation coefficient.

\section{Results}
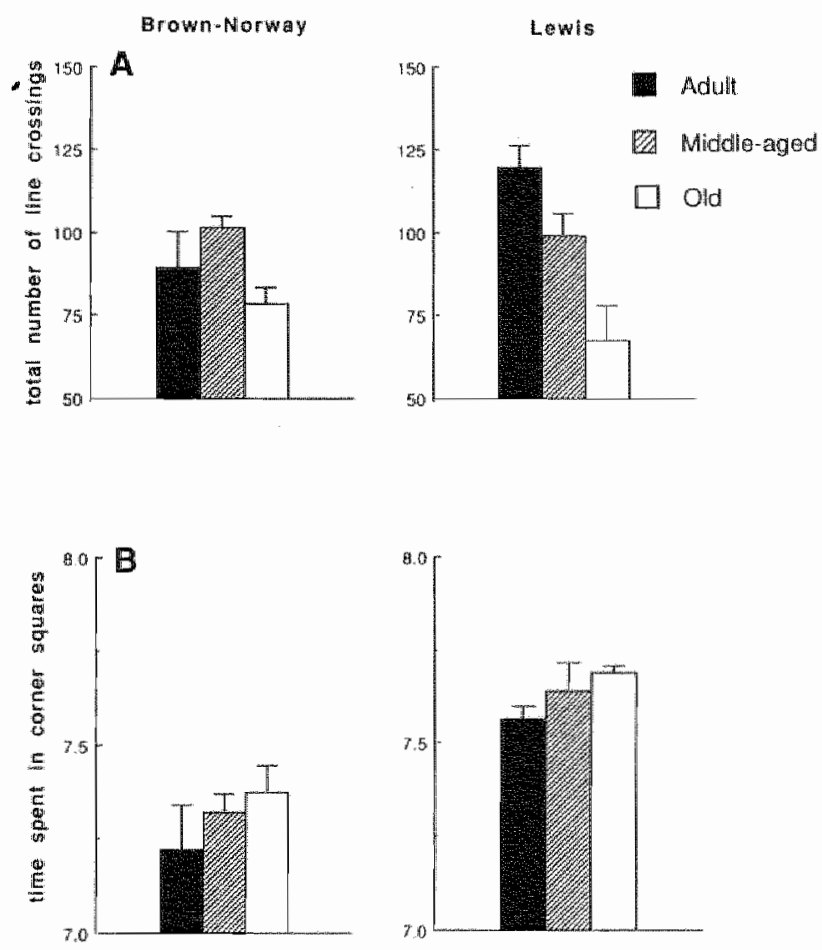

FIGURE 1: A) Total number of line crossings ( + SEM), and B) time spent in corner squares (in 0.1 is and transformed to the natural logarithm +SEM) of Brown-Norway (lleft panels) and Lewis (right panels) rats of three different ages in the open-field test. The data were aggregated over 4 daily sessions of 5 min. The aggregated measure, time spent in corner squares, was then transformed to the natural logarithm. 
In the OF test, the BN rats spent less time in the corner squares than the LEW rats did $(\mathrm{F}(2,41)=25.06 \mathrm{p}<0.01$; see Fig. $1 \mathrm{~A})$, indicating that the $\mathrm{BN}$ rats were less emotionally reactive than the LEW rats. In both strains there was a similar agerelated increase in the time spent in the corner squares (age $x$ strain interaction effect: $F(2,41)<1$, ns); however, age affected the total number of line crossings differently (age $x$ strain interaction effect: $F(2,46)=4.26, p<0.05$; see Fig. $1 B$ ). Correlation analysis revealed that the two measures of $O F$ behavior were correlated in the adult rats (LEW: $r=-0.79, p<0.05 ; \mathrm{BN}: \mathrm{r}=-0.87, \mathrm{p}<0.05$ ) but not in the middle-aged and old rats (for separate age groups within the BN and LEW strain: $0.13>r^{\prime} s>-0.61$, ns).
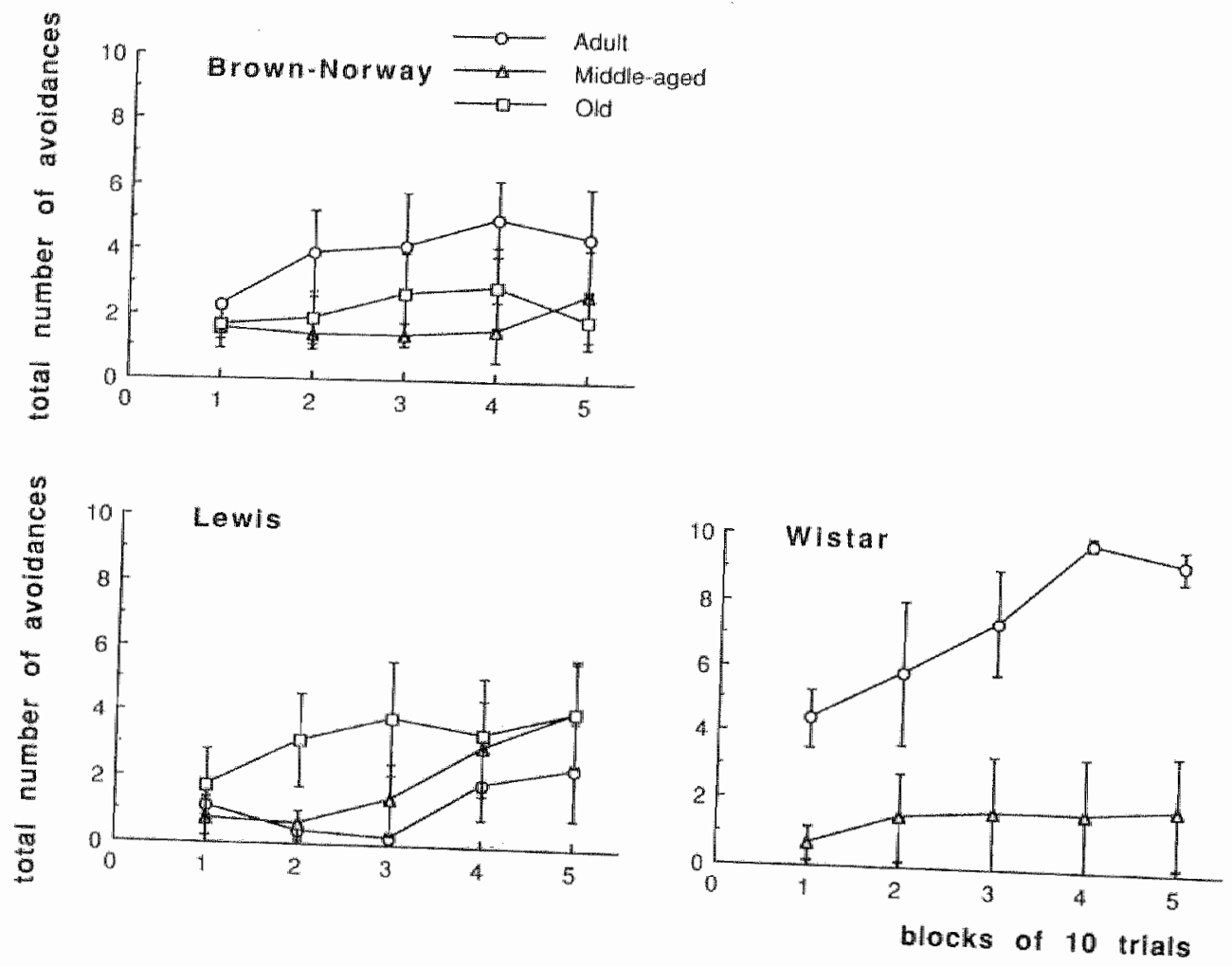

FIGURE 2: Number of a voidances (ISEM) of Brown-Norway (A), Lewis (B), and Wistar (C) rats of different ages during acquisition of a two-way active avoidance task in a shutle box.

Analysis of the acquisition of avoidance learning in the SB revealed that the general performance during acquistion was differentially affected by age and 
strain (general mean, age \& strain interaction effect: $F(3,50)=4.08, p<0.05$; see Fig. 2). When the acquisition rate of $S B$ learning was analysed within strains, it was found that the BN rats did not improve their performance ( $F^{\prime} s<2.02$, ns), and that the number of avoidance responses was very low for all age groups. In contrast, LEW rats improved their performance during acquisition $(F(4,80)=3.48$, $\mathrm{p}<0.05$ ). Old LEW rats learned the SB task faster than the younger rats lage $\mathrm{x}$ block interaction effect: $F(8,80)=3.03, p<0.01)$. The WIS rats learned to avoid the shock during acquisition (block effect: $F(4,36)=4.80, p<0.05)$. However, unlike the BN and LEW rats, the young WIS rats acquired the SB task faster than the old WIS rats (age effect for linear increase: $F(1,9)=5.97, p<0.05$ ). At the end of training, the young WIS rats showed an almost perfect avoidance performance.

\section{Discussion}

Strain differences were found in both the OF test and the SB task, which is in accordance with studies reporting strain differences in the behavioral response to stressful stimuli and acquistion of avoidance learning (Gilad \& Shiller, 1989; McCarty \& Kopin, 1978). Furthermore, this study showed that aging differentially affected the activity measure in the $\mathrm{OF}$ and that the two OF measures were not correlated in middle-aged and old rats. Corroborating earlier findings, the two OF measures were correlated in young rats (van der Staay et al, 1990b). This suggests that the novel environment elicited a different behavioral response in the different age groups of rats of the BN and LEW strains studied. The data from the SB experiment indicated that active avoidance learning was affected by strain and age. No age-related difference in SB performance was found in the BN rats. A similar poor avoidance performance of adult $\mathrm{BN}$ rats has also been reported by others (Gilad \& Shiller, 1989; van der Staay 1989). However, we found an agerelated increase in the rate of learning in the LEW rats. This contrasts with the generally found decline in learning and memory performance with aging. There was an age-related decrease in SB performance in the WIS rats, with the young rats performing almost perfectly, which indicates that rats can acquire the $S B$ task used in the present study. Because no consistent effect of age on SB performance was found for the different rat strains, it is conceivable that SB performance might be affected by age-dependent changes in mon-cognitive behavioral characteristics, one of which might be the response to a stressful stimulus. Since learning of the SB task is largely dependent on the animal's reactivity to shock, we hypothesize that reactivity to the footshock of the SB task is strain-and agedependent.

It could be argued that the age-related increase in shock intensity could explain the results of the SB experiment. However, we found that the shock sensitivity of young rats is approximately $0.075 \mathrm{~mA}$ and that the shock sensitivity of old rats is about $0.15 \mathrm{~mA}$. Thus the shock intensity in the SB experiment is well above the level of shock sensitivity. Furthermore, since the increase in the perceived intensity for physiological stimuli is logarithmically, we assumed that the age- 
related increase in shock intensity levels used in this study would lead to a comparable reinforcement value accross age groups.

In this study the rats were subjected to one SB training session of 50 trials. It could be argued that $S B$ performance of adult and middle-aged rats will improve after a first session and that a consistent age-related performance deficit can be found in subsequent training sessions. However, we have found that in the SB task of this study the performance of LEW and BN rats does not improve in a subsequent session of 50 trials (unpublished data).

A reduced locomotor activity of old rats (Gage et al, 1984a) could have affected SB performance. Howewer, we adjusted the parameters of the SB task to control for this possible confounding factor. The rats had to cross the barrier within $8 \mathrm{~s}$ after the onset of the warning signal which makes it unlikely that SB performance of old rats was dependent on an age-related decrease in motor performance. On the other hand, a reduced locomotor activity of old rats could have affected the operationalized measures of OF behavior. We have discussed this possibility in another study in which we concluded that emotionality is possibly expressed differently in young and old rats (Blokland et al., 1990).

Although reactivity to novelty and footshock may have distinct neuronal substrates, the results of both the OF test and SB task are indicative of a strain- and age-related change in the unlearned behavioral response to stressful stimuli. In line with the conclusion of Gilad and Shiller (1989), namely that the degree of reactivity to stressful environmental stimuli may affect learning, we conclude that age-related behavioral changes to stressors may also affect learning.

In summary, the present study showed that behavioral reactivity to novelty and avoidance learning was strain-dependent. In addition, we found that aging differentially affected measures which are related to the behavioral response to stressors. These findings suggest that age-related differences in performance in aversively motivated learning tasks (e.g., shock, water, novelty) may be confounded by age-related changes in the unlearned behavioral response to stressful stimuli. 


\title{
AGE DIFFERENCES IN FOOD MOTIVATION IN BROWN-NORWAY RATS
}

\author{
A. Blokland and W. Raaijmakers
}

\begin{abstract}
Although it has been recognized that the age differences in body weight could lead to age differences in food motivation, virtually no attempt has been made to evaluate food motivation in relation to age. In this study we attempted to compare food motivation in rats of three different ages under two different levels of food deprivation by measuring running speed in a runway. In addition, food motivation was measured in a progressive ratio schedule of reinforcement in an operant chamber at one level of deprivation. The resuits indicated that a differential food deprivation technique, i.e., an age-related increase in the percentual reduction in body weight, may be considered a reliable manner to achieve comparable levels of food motivation in rats of different ages.
\end{abstract}

\section{Introduction}

In what were presumably the first studies which evaluated the age-related differences in learning in rats, Stone (1929) recognized that there could be a problem with respect to different levels of motivation, because the body weight of old rats is normally higher than that of young rats. Williams \& Campbell (1961) showed that the percentual decrease in the free-feeding body weight of rats was related to their acceptance of quinine in their food. However, these studies evaluated the need for food rather than food motivation and did not involve rats older than 100 days.

In later studies on age-related differences in learning, Goodrick $(1968,1972)$ introduced a differential deprivation technique in food-motivated learning tasks in order to equalize the motivational level in young and old rats. Differential food deprivation holds that the percentual reduction in the free-feeding weight is related to age: the older the animal the greater the percentual reduction in body weight. Although the rationale behind the differential deprivation technique seems valid, most aging studies have been performed without using a differential deprivation technique, which controls for possible age-related differences in motivation (e.g. Wallace, Krauter \& Campbell, 1980; Hamm, Knisely, \& Dixon, 1983; Dunnett, Evenden, \& Iversen, 1988). A deprivation technique that has often been used is to allow the subjects to eat/drink for a fixed time per day. However, Campbell et al. (1961) showed that this may not be an appropriate manner to establish comparable levels of deprivation in rats of different ages (see also Elias \& Elias, 1977). One reason why a differential deprivation technique has not often been used in aging studies is that there are few studies which have evaluated age-related differences in motivation.

Van der Staay et al. (1990d) applied a differential deprivation technique in a study which evaluated the age-related decline in spatial discrimination learning 
in a holeboard task in Brown-Norway rats. They found, that in the course of training, spatial discrimination performance diverged between age groups whereas the speed of responding converged. Thus, although the old rats had an impaired spatial discrimination performance, the speed of responding was not different between the age groups at the end of training. These findings were considered to support the use of a differential deprivation technique when comparing subjects of different ages in learning tasks. However, to our knowledge, there have been no studies which systematically evaluate age-related differences in food motivation by applying a differential food deprivation technique.

Different paradigms have been used to study motivational behavior. In a runway, the running speed of the subjects is taken as a measure of motivation: the higher the running speed, the higher the food motivation. In a progressive ratio schedule of reinforcement (Hodos, 1961; Van Hest et al., 1988), the number of lever presses the rat is willing to make in order to receive a reward is taken as a measure of motivation. Both tasks have a strong motoric component. It has been found that motor performance generally declines with age in rats (e.g., Gage, Dunnett, \& Björklund, 1984a), which may complicate the evaluation of agerelated differences in motivation when these measures are used.

In the present study we evaluated food motivation, using a runway, in BrownNorway rats of three different ages at different deprivation levels $(90 \%$ and $80 \%$ of the free-feeding body weight). We tested the rats twice at both deprivation levels: first in an ascending order (from $90 \%$ to $80 \%$ ) and then in a descending order (from $80 \%$ to $90 \%$ ). This offered the possibility to evaluate the percentual increase or decrease in running speed within subjects (age groups) as a measure of food motivation. Within-subject analysis rules out bias as a result of differences in motor performance. We used Brown-Norway rats for two reasons. Firstly, this strain is free of specific age-associated pathologies (Burek, 1978). Secondly, we found that the speed of responding reached comparable levels in young and old rats in a holeboard experiment (van der Staay et al., 1990d), indicating that, up to an advanced age, there are no gross motor dysfunctions in this strain. This minimizes age differences in the speed of responding. We also subjected the rats to a progressive ratio schedule of reinforcement in an operant chamber at an $80 \%$ deprivation level.

\section{Materials and methods}

Animals.

We used male Brown-Norway (BN) rats of three different ages ( 8 months, $n=$ 7; 14 months, $\mathrm{n}=8 ; 26$ months, $\mathrm{n}=7$ ). All rats were housed individually in standard Makrolon cages on sawdust bedding in an air-conditioned room (about $20^{\circ} \mathrm{C}$ ). They were kept under a $12 / 12$ hour light dark cycle (lights on from 9:00 to 21:00). 
Food deprivation.

The body weight of the rats was gradually reduced to $90 \%$ of the free-feeding weight before the rats were subjected to the first series of tests. After the first test series, the body weight of the rats further reduced to $80 \%$ of their free-feeding weight. The rats were given food ad libitum for three weeks after they had completed the second series of tests. Then the free-feeding weight was determined again. This weight was used as the reference value for the subsequent deprivation period. After the third tests series, in which the body weight of the rats was gradually reduced to $80 \%$ of their free-feeding weight, the rats were allowed to gain weight up to $90 \%$ of their free-feeding weight. Then the final series of tests was carried out.

\section{Procedures.}

Runway. The runway $(245 \times 12 \times 15 \mathrm{~cm})$ was made of lacquered wood. A polyvinylchloride goal box with a food tray was connected to it. A metal obstruction could be placed at any place in the runway to adjust the length of the runway.

Before testing, the rats were given a total of 23 adaptation trials in the runway. A rat was left in the goal box until it had consumed the food reward (six 45-mg Bioserve food pellets). A rat was always started with its head away from the goal box. In adaptation sessions the length of the runway was gradually increased from $20 \mathrm{~cm}$ to $120 \mathrm{~cm}$. During training trials the length of the runway was 120 $\mathrm{cm}$. Special attention was given to keep test conditions constant, e.g., background noise, similar testing procedures, same position of runway in the room. The runway was cleaned after each session. All testing was carried out in a darkened room. Per session a rat was given three trials in succession. A test series consisted of a total of 5 sessions of three trials. The latency to enter the goalbox with four paws was measured.

Progressive patio. The experiments were carried out in four identical conditioning chambers $(40 \times 30 \times 33 \mathrm{~cm})$. The ceiling of the Skinner boxes contained a light that illuminated the conditioning chamber during the experiments. The left and right side-walls served as control panels and included manipulanda and discriminanda. A recess $(5 \times 5 \mathrm{~cm})$ was built into the left sidepanel $2.5 \mathrm{~cm}$ above the grid floor and contained a food tray, into which a pellet dispenser delivered $45 \mathrm{mg}$ food pellets (Bioserve). Two retractable stainless steel levers ( $4 \mathrm{~cm}$ wide) projected $2 \mathrm{~cm}$ into the Skinner box. The levers were located 6 $\mathrm{cm}$ from both sides of the recess, $12 \mathrm{~cm}$ above the grid floor. The conditioning chambers were enclosed in sound-attenuating housing. An exhaust fan produced background noise. An MS-DOS microcomputer controlled the experimental equipment and collected the data.

Training and testing on the progressive ratio schedule in a conditioning chamber was carried out during the second test series. The rats received two 30minute magazine training sessions before they were trained on a continuous reinforcement schedule to press the left lever on the left wall. After the rats had acquired the lever press response, they were subjected to the progressive ratio 
schedule (PR-5, 5 refers to the incremental steps of lever presses necessary to obtain one food pellet) to evaluate food motivation in two sessions. A PR-5 session was ended when the time between two lever presses exceeded 10 minutes. The total number of lever presses during a session was measured.

\section{Statistical analysis.}

Runway. The mean time to enter the goal box was calculated for each test session according to the formula $\left(t_{1}+t_{2}+t_{3}\right) / 3$ (the subscript refers to the trial). The mean running speed per session was calculated by dividing 120 (length of runway) by the mean run time. The mean running speed was calculated for the five test sessions. For data analysis the running speed was transformed logarithmically to remove inhomogeneity.

We first evaluated whether there was a percentual increase $(90 \%$ to $80 \%$ deprivation level) or decrease $(80 \%$ to $90 \%$ deprivation level) in running speed by using t-statistics. Differences in food motivation were assessed by comparing the mean percentual decrease in latency from test series $1(90 \%)$ to $2(80 \%)$ and the percentual increase from test series $3(80 \%)$ to $4(90 \%)$ within subjects in a one-factorial (age) analysis of variance. A Duncan-Waller post-hoc analysis evaluated the age-related differences in more detail. Differences in running speed at similar deprivation levels were evaluated by using t-statistics.

Progressive ratio. The mean number of lever presses during the two PR-5 sessions was calculated and transformed logarithmically to remove inhomogeneity. The transformed measure was analyzed in a one-factorial (age) analysis of variance. A Duncan post-hoc analysis evaluated the data in more detail.

Correlational analysis. The data from the runway and PR-5 test were correlated using Pearson's correlation coefficients.

\section{Results}

The free-feeding body weight of the animals is shown in Table 1.

Table I: Free-feeding body weight (mean in g (SEM)) of the Brown-Norway rats of different ages at the start of the two deprivation periods.

\begin{tabular}{lccc}
\hline & Young & Middle-aged & Old \\
13t period & $334(5.6)$ & $395(9.9)$ & $478(13.7)$ \\
$2^{2 n d}$ period & $367(7.1)$ & $404(8.6)$ & $463(13.5)$ \\
\hline
\end{tabular}




\section{Runway.}

The running speed of the old rats increased from the first to second test series $(t, 6)=6.79, p<0.01$; see Fig. 1). There was a tendency for an increase in rumning speed in young rats $(t(6)=2.12,0.10>p>0.05)$, but the running speed of the middle-aged rats was not affected by an increase in the level of food deprivation $(t(7)=0.32$, n.s.). An analysis of variance showed that there was weak statistical support for the existence of an age-related difference in the percentual increase in running speed $(F(2,21)=3.27,0.10>p>0.05)$. The percentual increase was similar in young and old rats. From test series 3 to 4 , the running speed of rats from the three age groups showed a decrease $(t>3.08, p<0.05)$ that was similar for all age groups $(\mathrm{F}(2,21)<1$, n.s.). Evaluation of the running speeds at similar deprivation levels revealed that only the running speed of the old rats was higher during the first test series than during the fourth test series, both at the $90 \%$ level $(t(6)=2.98$, $p<0.05)$.

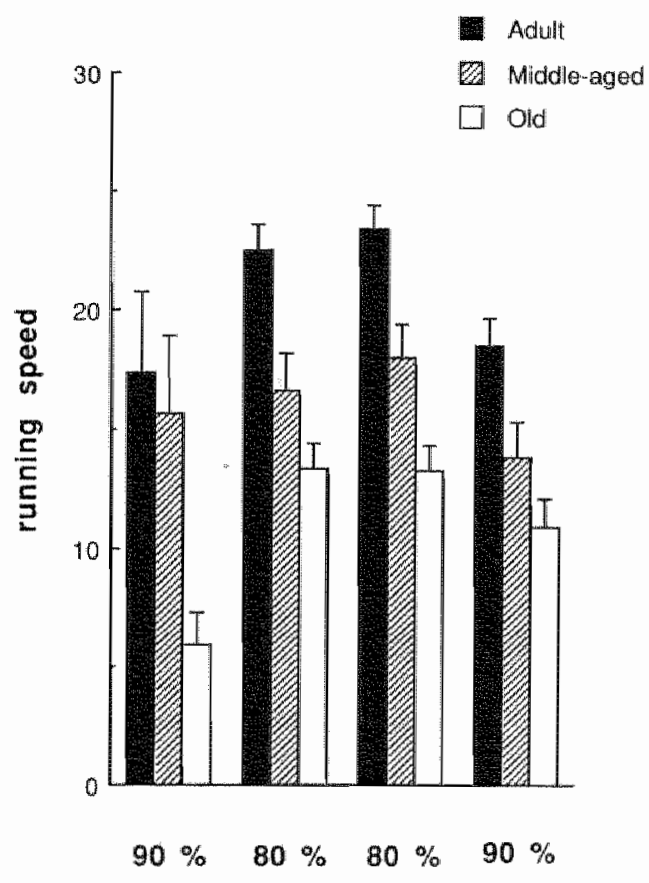

FIGURE 1: Running speed (cm/s) in a rumway of adult, middle-aged, and old Brown-Norway rats in a runway in 4 test series each consisting of 5 sessions of 3 trials. Two different food deprivation levels werre asessed.

\section{Progressive ratio.}

There was an age-related decrease in the number of lever presses during the PR-5 sessions $(\mathbb{F}(2,21)=5.29, \mathrm{p}<0.01$; see Fig. 2$)$. Post-hoc analysis showed that the adult and middle-aged rats pressed the lever more often than the old rats. 
Correlational analysis.

All correlations among the four different mean running speeds in the runway were found to be highly correlated $(r>0.69$, all associated probabilities $<0.01$ ). No correlations were found between mean running speeds during the four runway sessions and PR-5 performance ( $r^{\prime} s<0.38$, n.s.).

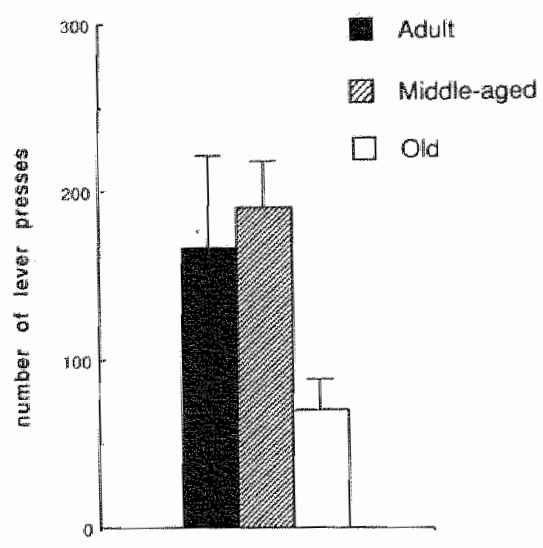

FIGURE 2: Mean number of lever presses of adult, middle-aged, and old Brown-Norway rats in two sessions of progressive ratio schedule of reinforcement in a conditioning chamber.

\section{Discussion}

In the first test series the running speed of the old rats in the runway was three times lower than that of the middle-aged or young rats. The age-related difference in running speed was much smaller when the deprivation level was $80 \%$ when the running speed of the old animals was comparable to the running speed of the young rats at the $90 \%$ deprivation level. The percentual increase in running speed from test series 1 to 2 was equal in young and old rats whereas the middleaged rats did not increase their running speed. This indicates that food motivation was increased similarly in young and old rats during the first two test series. The decrease in running speed from test series 3 to 4 was also found to be similar. The age-related differences in motor performance were minimized by using a short runway and Brown-Norway rats. The finding that the running speed of old and young rats was more comparable when the percentual decrease in body weight of the old rats was greater than that of young rats $(80 \%$ versus 90 $\%$, respectively), suggests that a differential food deprivation technique may be considered as a method to achieve comparable levels of food motivation in rats of different ages.

Recently, Ando and Ohashi (1991) showed that the level of food deprivation predicted the percentage of good learners in a learning task differently in young 
and old rats. The percentage of good learners was similar in young and old rats when the body weight of the old rats was reduced more than that of the young rats. Although they concluded that this indicated that a differential food deprivation technique should be used, their data should be interpreted with caution. Their assumption that the level of food deprivation is related to learning performance may not be completely correct since food motivation may depend on various processes (Bolles, 1975a).

The observation that the old rats had lower running speeds during the first than during the fourth test series, when deprivation levels were equal, suggests that the operationalized measures of food motivation, as measured in the runway, may have a component(s) that interferes with the measure running speed. One factor that could account for this is that old rats need more training to achieve a maximal level of performance. This is supported by the finding that the speed of responding of different age groups of Brown-Norway rats converges during training in a learning task and that old rats increase their speed of responding faster than animals of other age groups (van der Staay et al., 1990d). It is conceivable that age-related differences during the first phase of learning in learning tasks may be confounded by age-related differences in the time needed to achieve maximal levels of responding.

The notion that learning may affect the interpretation of data obtained from experiments assumed to measure motivation (Bolles, 1975a) was supported by the poor correlation between the data from the runway and the PR-5 test. Apparently, the two different operationalizations of food motivation measure different aspects of food motivation. This may be explained by the different task aspects which might well be differently sensitive to motivational changes (e.g., Bolles, 1975b). Such aspects could be: difference in the response to obtain a reinforcement (rate measures on the one hand and speed or latency measures on the other), amount of reinforcement per response, and test environment.

In summary, the runway experiment suggest that a differential food deprivation technique may be regarded as an appropriate technique to achieve equal levels of food motivation in rats of different ages. Assessment of food motivation was test-dependent, which suggests that food motivation is not a unitary concept and that other factors may interfere with motivation. In turn, the interaction between learning and motivation is complex and should be controlled, especially in studies in which differences in motivation are likely to influence performance, e.g. aging studies.

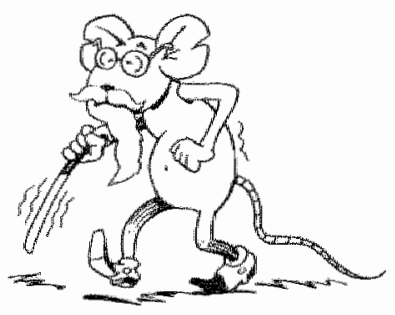





\title{
CORRELATION BETWEEN BEHAVIORAL AND BIOCHEMICAL PARAMETERS IN YOUNG AND OLD LEWIS RATS
}

\author{
Arjan Blokland
}

\begin{abstract}
This study evaluated aging by using a within subject analysis of behavior in young (4-month-old) and old (24-month-old) rats. The rats were subjected to different learning tasks and non-cognitive tests and the relation between parameters was assessed. In the learning tasks (spatial and temporal discrimination), except for the inhibitory avoidance task, the first part of the learning curve was taken as an index of learning. There was no correlation between the different parameters of learning in young and old rats. This indicates that there are individual differences in performance in different learning and memory tasks. The results also suggest that different learning tasks measure different (non)cognitive processes. Furthermore, it would appear that there are individual differences in the age-related decline in cognitive processes. Non-cognitive behavior (food motivation, emotional reactivity, motor performance) did not predict performance in the learning tasks. Blood glucose and hippocampal choline acetyltransferase (ChAT) activity were also measured. Hippocanpal ChAT activity did not correlate with learning performance in old rats whereas blood glucose was found to correlate with spatial learning in old rats These results suggest that an impaired regulation of blood glucose regulation may be a factor underlying the cognitive decline in aging.
\end{abstract}

\section{Introduction}

During the last decades age-related changes in biochemical and behavioral measures have received increasing attention in human and animal studies aimed at understanding the age-related decline in cognitive functions. Most of the studies have evaluated age differences in a cross-sectional design, in which subjects of different ages are compared. Another experimental set-up to study age-related differences is a longitudinal study, where subjects are tested at several points during their lifetime to evaluate individual changes during the aging process (e.g. Ando \& Ohashi, 1991; Caprioli et al., 1991). This approach may be more reliable than a cross-sectional approach as, for example, cohort effects can be excluded (see Ingram, 1985). Finally, individual differences in the aging process can be studied in a correlational study by assessing different parameters in one subject.

Relatively few studies have evaluated individual differences in aging (e.g. Barnes, 1979; Gage et al. 1984b, 1988; Markowska et al., 1989; Stone et al., 1990). Although such an approach has not been used often, these studies may contribute to our understanding of the process of aging (Olton \& Markowska, 1988). For example, the findings of Markowska et al. (1989) suggest that agerelated learning and memory impairments are correlated to changes in sleep pattern and brain chemistry. A correlational study, thus, provides information 
about within-subject variance and may reveal a relation between behavioral and/or biochemical parameters.

The aim of the present study was to evaluate the interdependence of a number of cognitive and non-cognitive behavioral parameters in young and old rats. We used young and old rats because a relationship between parameters within one age group would not indicate whether there is an age-related change in the relation between different measures. The rats were subjected to different learning tasks (Morris swim task, a spatial cone-field task. Fixed Interval 60-s schedule of reinforcement, inhibitory avoidance performance) to evaluate the relation between different forms of learning, i.e., whether poor performance in one learning task predicts poor performance in a task that measures another aspect of learning. Since the most dynamic change in learning occurs during the first phase of training, the first part of the learning curve was taken as an index for the different parameters of learning. Furthermore, we subjected the rats to different non-cognitive tests (emotional reactivity, bridge task, food motivation) to evaluate whether non-cognitive factors predict performance in learning tasks. The cognitive and non-cognitive behavioral measures were correlated with blochemical measures, namely baseline blood glucose levels, blood glucose regulation (as measured by the increase in blood glucose after an i.p. injection of glucose), and hippocampal choline acetyltransferase (ChAT) activity. These biochemical markers were selected because they may be related to cognitive function. A poor regulation of blood glucose (Hall et al., 1989; Stone et al., 1988) and a decrease in ChAT activity (Bartus et al., 1982) have both been found to correlate positively with a decrease in cognitive function.

The young and old rats were tested in two separate experiments and therefore no attempt was made to compare the age groups directly. We only evaluated the age-related differences in the correlation between parameters. We compared age groups onle when evaluating the difference in variation of the behavioral measures. This was done to evaluate whether the variance in performance in the tasks differed between young and old rats.

\section{Materials and methods}

\section{Animals}

We used eighteen 4-month-old and twenty-two 24-month-old male Lewis rats (range, 23-25 months). None of the old rats had cataracts. The rats had no motor impairments and were physically able to perform the responses which were required in the different experiments. All rats were housed individually in standard Makrolon cages on sawdust bedding in an air-conditioned room (about $20^{\circ} \mathrm{C}$ ). They were kept under a 12/12 hour light-dark cycle (lights on from 9:00 to 21:00). During training four old rats died for unknown reasons and one young rat was excluded from behavioral testing because it suffered from paralysis of the hind legs. 


\section{Procedures}

The young and old rats were subjected to the different behavioral tests (procedures) in the same order (see Table 1). All testing was carried out between 9:00 and 15:00.

T.ABLE I: Order and duration of behavioral experiments/procedures.

\begin{tabular}{ll}
\hline week 1. & Morris swim task. \\
week 2. & Open field test. \\
week 3. & Start food deprivation. \\
week 4. & Adaptation cone-field task. \\
week 5-7. & Acquisition training cone-field task. \\
week 8. & Inhibitory avoidance task and bridge task. \\
week 9. & Magazine and continuous reinforcement training in Skiniver box. \\
week 10-11. & Progressive ratio test and fixed-interval 60 s training. \\
week 12. & Blood glucose (baseline/peak level) determination and decapitation.
\end{tabular}

Morris swim task: The rats were trained on the standard Morris spatial navigation task (Morris, 1981) in a black water tank (diameter $1.22 \mathrm{~m}$ ). The rats were started from four different, randomly chosen start positions and trained to find an invisible platform (diameter $11 \mathrm{~cm}$ ) that was at a fixed position in the water tank, $1 \mathrm{~cm}$ below the surface of the water. The temperature of the water was $20-22^{\circ} \mathrm{C}$. A trial lasted until a rat had found the platform or until $60 \mathrm{~s}$ had elapsed. If a rat did not find the platform within $60 \mathrm{~s}$ it was placed on the platform for $3 \mathrm{~s}$ and then removed from the water tank. On the first day the rats were given four trials. The rats were given eight trials per day on consecutive days. The young rats were trained to a total of 20 trials whereas the old rats were tested to a total of 28 trials.

Open-field test: The open-field consisted of a square base $(100 * 100 \mathrm{~cm})$ subdivided into 36 equal squares by black lines (for details see 15). Two red fluorescent tubes provided very dim illumination (about 2 lux) on the floor of the apparatus. Immediately after a rat was placed in the center of the OF, the movements of the rat were scored. A crossing was scored as soon as the rat crossed a line with both hind legs. The total number of lines crossed and the total time spent in the corner squares were recorded manually wilth an MS-DOS microcomputer. The experimenter sat in front of the OF. Testing was carried out on 4 consecutive days in 5 -min sessions.

Spatial cone-field task: The cone-field apparatus, which is fully automated, has been described in detail elsewhere (van der Staay et al., 1990a, 1990c). The experimental room was illuminated by four red fluorescent lights and three 100W'bulbs, which were adjusted by a dimmer to give an intensity of about 50 lux on the floor of the apparatus. Extra-maze cues consisted of two doors, a one-way screen and a window (both covered by black curtains), a sink, and two tables, on 
one of which was a computer and interface. The experimenter sat in front of the apparatus. He was present and visible throughout the behavioral testing. We applied a differential deprivation technique (cf. Goodrick, 1972) and reduced the body weight of the rats to $85 \%$ (young) and $77.5 \%$ (old) of their free-feeding values.

Seven days after the start of food deprivation, the rats were familiarized with the cone-field in 10-min sessions on 4 consecutive days. All cones contained one 45-mg food pellet (Bioserve) during the adaptation sessions.

After the adaptation sessions, the rats were trained in the cone-field with massed trials (day 1, two trials; days 2-4, three trials; day 5, four trials; days 6-13, six trials). The young rats were trained to a total of 50 trials and the old rats to a total of 60 trials.

Four cones of a fixed set contained one food pellet each. A trial was started by placing the rat in the start box. The sliding door was then opened immediately. As soon as the rat had entered the cone-field, the sliding door was closed. Whenever the rat touched the top of a cone, it closed an electrical circuit and activated a counter. This was scored automatically as a cone visit. Only contacts preceded by a visit to another cone were scored (excepting the first cone visit). Infrared photocells detected whether a rat poked its nose into the top of the cone. An MS-DOS microcomputer collected the data and controlled the duration of the trials. A trial was terminated when the rat had found and consumed all four food pellets or when $10 \mathrm{~min}$ had elapsed, whichever event occurred first. The animal was put back into its home cage between trials. After the cone-field had been cleaned with a damp sponge, and the four cones had been re-baited, the next trial was started.

Inhibitory avoidance: The inhibitory avoidance apparatus consisted of a light and a dark compartment, each measuring $40 \times 25 \times 40 \mathrm{~cm}$. The light compartment was illuminated (about 1000 lux) by a light bulb mounted above the compartment. The floor consisted of a metal grid connected to a shock scrambler. The two compartments were separated by a guillotine door that could be raised $10 \mathrm{~cm}$. On day 1 the rats were placed in the dark compartment and removed from the apparatus after 2 min. After 2 min the rats were placed in the light compartment and the latency to enter the dark compartment with four paws was measured. The guillotine door was lowered and the rats were left in the dark compartment for $10 \mathrm{~s}$ before they were removed from the apparatus. On day 2 the rats were given three trials, which were similar to the second trial on day 1 . In the third trial, however, a scrambled shock ( $2 \mathrm{~s}, 0.25 \mathrm{~mA}$ (young rats) / $0.30 \mathrm{~mA}$ (old rats)) was administered via the grid floor after the guillotine door was lowered. The old rats were given a higher shock intensity because of the age-related decrease in shock sensitivity (unpublished data). On the third day, after a 24-h interval, the retention performance was measured as the latency to enter the dark compartment with four paws. The old rats were left in the light compartment to a maximum of $600 \mathrm{~s}$. In order to increase the behavioral variance in young rats, young rats were left in the light compartment to a maximum of $900 \mathrm{~s}$. 
Bridge task: A rat was placed on the center of a plank, $2.5 \mathrm{~cm}$ wide for the young rats and $5 \mathrm{~cm}$ wide for the old rats, $30 \mathrm{~cm}$ above a table. The latency to fall from the bridge was measured, with a maximum of $60 \mathrm{~s}$, in two trials given in succession.

Skinner box tasks: All Skinner box tasks were carried out in four identical conditioning chambers $(40 * 30 * 33 \mathrm{~cm})$. The ceiling of the Skinmer boxes contained a light that illuminated the conditioning chamber during the experiments. The left and right side-walls served as control panels and included manipulanda and discriminanda. A recess $(5 \times 5 \mathrm{~cm})$ was built into the left side panel $2.5 \mathrm{~cm}$ above the grid floor and contained a food tray, into which a pellet dispenser delivered 45 - $\mathrm{mg}$ food pellets (Bioserve). Two retractable stainless steel levers ( $4 \mathrm{~cm}$ wide) projected $2 \mathrm{~cm}$ into the conditioning chamber and were located $6 \mathrm{~cm}$ from both sides of the recess, $12 \mathrm{~cm}$ above the grid floor. The conditioning chambers were enclosed in sound-attenuating housing. An exhaust fan produced background noise. An MS-DOS microcomputer controlled the experimental equipment and collected the data.

The rats received two 30-min magazine training sessions before they were trained on a continuous reinforcement schedule to press the left lever on the left wall. After the rats had acquired the lever press response, the rats were subjected to a progressive ratio schedule (PR-5, 5 refers to the incremental steps of lever presses to obtain one 45-mg Bioserve food pellet) to evaluate food motivation in two sessions. A higher level of food motivation is operationalized as a greater number of lever presses during a PR-5 session (Hodos, 1961). A PR-5 session was ended when the time between two lever presses exceeded $10 \mathrm{~min}$. Temporal discrimination training in a fixed-interval 60 -s schedule started 1 day after the PR5 session. The rats were subjected to 10 daily 1 -h FI-60 sessions.

Blood glucose: Blood glucose level and blood glucose regulation were determined in one experiment. After a rat was placed in a fixation cage an incision was made in the tail vein. Blood was collected in a heparinized capillary vial. After the rats were removed from the fixation cage, the rats received an injection of a glucose (500 $\mathrm{mg} / \mathrm{kg}$ glucose dissolved in distilled water, i.p.). Blood samples were collected 10, 30, 60 and $120 \mathrm{~min}$ after injection. Blood glucose levels were measured using a commercial kit (Gluco-quant, Boehringer-Mannheim).

Choline acelyltransferase: The rats were decapitated and the hippocampus was dissected at $4^{\circ} \mathrm{C}$. Tissue samples were stored at $-80^{\circ} \mathrm{C}$ until used for measure of ChAT activity. Brain samples were homogenized $(5 \%)$ in a $50 \mathrm{mM}$ phosphate buffer (pH 7.4) and combined with $0.2 \%$ Triton $X-100$ and 20 mM EDTA (pH 7.4). ChAT activity was determined according to the method of Fonnum (1975), using ${ }_{14} \mathrm{C}$-acetyl coenzyme-A (Amersham, $50-60 \mathrm{mCi} / \mathrm{mM}$ ).

\section{Statistical analysis}

In the different learning tasks (except for inhibitory avoidance) we arbitrarily 
operationalized learning as the increase in performance during the first phase of training, which corresponded to the part of the learning curve one trial block before it started to flatten. We analyzed the slope of the learning curves by calculating the trend component and the height of the part of the curve for which the trend component was calculated. The trend component which explained most of the variation in the increase in learning was taken as a parameter of the increase in learning.

Correlations between the different parameters were analyzed using Pearson's correlation coefficient $\left(\mathrm{r}_{\mathrm{p}}\right)$. Where different parameters contributed substantially to an operationalization of an index (for example, general mean (i.e., height of learning curve), and orthogonal trend component (i.e. slope of learning curve) for learning), the canonical correlation $\left(r_{c}\right)$ between the sets of variables was analyzed. The canonical correlation procedure finds a linear combination for each set (referred to as a canonical variable) in such a way that the correlation between the two canonical variables is maximized (SAS User's Guide, Statistics, V5).

The parameters chosen for statistical analysis were the following: Morris swim task: The mean escape latency for blocks of four trials was calculated. Orthogonal trend components were calculated over the first three and four (for young and old rats, respectively) trial blocks (Winer, 1971), which correspond to the first phase of training for each age group. The general mean and the trend component over these first trial blocks were taken as parameters for correlational analysis.

Open-field test: The data of the open-field test were aggregated in order to enhance reliability (Ossenkop \& Mazmanian, 1985). The mean time spent in the corner squares and the mean total number of line crossings were taken as parameters of emotional reactivity (see van der Staay et al., 1990b).

Cone-field task: The measures working memory (WM) (number of rewarded visits / number of visits to the baited set of cones) and reference memory (RM) (number of visits to the baited set of cones / number of visits to all cones) were taken as indices of spatial discrimination performance in the cone-field task. The means for both variables were calculated for blocks of 10 trials. Orthogonal trend components were calculated over the first three trial blocks for WM and over the first four trial blocks for RM for both age groups. These trial blocks correspond to the first phase of spatial discrimination learning for both age groups. The number of trial blocks over which trend components were calculated was different for WM and RM because the learning curves differed, i.e, WM performance was found to reach an asymptote at an earlier stage. For both WM and RM the general mean and the trend component over these first trial blocks were taken as parameters for correlational analysis.

Inhibitory awoidance: The step-through latency in the 24-retention session minus the step-through latency during the shock trial was taken as a parameter of inhibitory avoidance performance.

Bridge task: The mean latency to fall in the two sessions was calculated and was taken as a parameter of sensorimotor function.

Food motivation. The mean number of lever presses during the two PR-5 
sessions was taken as the parameter for food motivation.

Fixed-interval 60 s: The interval of $60 \mathrm{~s}$ of the FI 60 schedule was divided into six classes of $10 \mathrm{~s}$. The mean curvature index (Fry et al., 1960) was calculated per block of two daily sessions and was taken as an index of temporal discrimination learning. The general mean and the trend component were calculated over the first three session blocks and were taken as parameters of temporal discrimination learning. The first three trial blocks correspond to the first phase of the acquisition of the FI 60 task.

Blood glucose: The blood glucose level before injection was taken as the baseline glucose level. The blood glucose regulation parameter was operationalized as the peak level after injection: the higher the peak level after injection the worse the regulation of blood glucose was.

Choline acetyltransferase: The value of hippocampal ChAT activity was taken as the parameter of hippocampal cholinergic activity.

Finally, it was evaluated whether the variance in performance in the different behavioral tasks (mean and trend component of the Morris task, time spent in corner squares and total number of line crossings in the open-field test, WM and RM in cone-field task, inhibitory avoidance (for the young rats the time was corrected by using the maximum of $600 \mathrm{~s}$ to achieve comparable testing conditions), progressive ratio, and fixed-interval $60 \mathrm{~s}$ ) was different for young and old rats. The standard errors of means of the behavioral measures of the different tasks were analyzed by comparing the percentual difference (1 - (SEM(young)/ SEM(old) $x$ 100) using a t-test. It must be noted that the comparison between the variances can only be considered as an explorative analysis because the data from the young and old rats stem from two different experiments.

\section{Results}

\section{Correlation between learning parameters}

The increase in performance in the different tasks was predominantly characterized by a linear trend component for both age groups (see table II). Consequently, the linear trend component was taken as an index for the rate of learning. The parameters of learning (Morris swim task, cone-field task, inhibitory avoidance, and fixed-interval $60 \mathrm{~s}$ ) were not correlated in either young rats (all canonical correlations: $r_{c}<0.63$, Fs $<2.10$, n.s.) or old rats (all canonical correlations: $\mathrm{r}_{\mathrm{c}}<0.58$, Fs $<1.72, \mathrm{n.s}$.).

\section{Correlation among indices of emotionality}

The indices of emotional reactivity (total number of crossings and time spent in corner squares) were negatively correlated in young rats $\left(r_{p}=-0.86, p<0.01\right)$, but were not correlated in old rats $\left(r_{p}=-0.39, \mathrm{n.s}\right.$. $)$. 
TABLE II: Percentage of explained variance for the trend component in the different leaming tasks for young and old rats.

\begin{tabular}{|c|c|c|}
\hline & Young & Old \\
\hline Morris swim task & $\begin{array}{l}\text { linear trend } \\
79 \%\end{array}$ & $\begin{array}{c}\text { linear trend } \\
73 \%\end{array}$ \\
\hline $\begin{array}{l}\text { Cone-feld task: } \\
\text { working memory }\end{array}$ & $98 \%$ & $99 \%$ \\
\hline $\begin{array}{l}\text { Cone-field task: } \\
\text { reference memory }\end{array}$ & $92 \%$ & $99 \%$ \\
\hline Fixed-interval 60 s & $99 \%$ & $82 \%$ \\
\hline
\end{tabular}

Correlation between baseline glucose level and learning parameters

There were no correlations between the different learning parameters and baseline glucose level in young rats (all canonical correlations: $\mathrm{r}_{c}<0.44$, Fs $<1.70$, n.s.). However, in old rats there was a negative correlation between baseline glucose level and the linear increase in working memory $\left(r_{p}=-0.53, p<0.05\right)$ and reference memory $\left(r_{p}=-0.70, p<0.01\right)$ in the cone-field task. The canonical correlations $\left(r_{c} s\right)$ between baseline glucose level and the learning parameters for working memory and reference memory were $0.57(\mathrm{~F}(2,15)=3.72, \mathrm{p}<0.05)$ and $0.71(\mathrm{~F}(2,15)=7.77, \mathrm{p}<0.01)$, respectively. The other learning parameters did not correlate with the baseline glucose level in old rats (all canonical correlations: $r_{c}<$ 0.37 , Fs $<1.21$, n.s.).

Correlation between baseline glucose level and non-cognitive behavioral parameters

The baseline glucose level in young rats was negatively correlated with the mean number of lever presses during the PR-5 sessions: the higher the baseline glucose level the lower the motivation to press the lever $\left(r_{p}=-0.59, p<0.05\right)$. There were no other correlations between baseline glucose level and noncognitive behavioral parameters in young rats. There were no correlations between baseline glucose level and non-cognitive behavioral parameters in old rats $\left(\mathrm{r}_{\mathrm{p}}<0.35, \mathrm{n.s}.\right)$.

Correlation between peak glucose level and learning parameters

In young rats the peak level of glucose was positively correlated with inhibitory avoidance performance $\left(r_{p}=0.54, p<0.05\right)$. There were no other correlations between peak glucose level and learning parameters (all canonical correlations: $\mathrm{r}_{\mathrm{c}}<0.26, \mathrm{Fs}<0.51$, n.s.). In old rats the peak glucose level was negatively correlated with both parameters of reference memory in the cone-field task (general mean $r_{p}=-0.64, p<0.01$; linear trend component $r_{p}=-0.66, p<0.01$ ). 
The canonicall correlation $\left(r_{c}\right)$ between the peak glucose level and the learning parameters for reference memory was $0.69(\mathrm{~F}(2,15)=6.86, \mathrm{p}<0.01)$. The other learning parameters did not correllate with the peak glucose level (all canonical correlations: $r_{c}<0.47, F^{\prime} s<2.12$, n.s.).

TABLE II: Standard errors of mean (SEM) and the percentual differences for the different behavioral parameters for young and old rats (1 - (SEM(young) / SEM(old) $x$ 100).

\begin{tabular}{|c|c|c|c|}
\hline & SEM(Young) & SEM(OId) & $\begin{array}{l}\text { percentual } \\
\text { difference }\end{array}$ \\
\hline $\begin{array}{l}\text { Morris swim task: } \\
\text { general mean }\end{array}$ & 2.50 & 4.20 & $40.4 \%$ \\
\hline linear trend & 1.98 & 2.07 & $4.2 \%$ \\
\hline \multicolumn{4}{|l|}{ Open field test: } \\
\hline Time in comer squares. & 0.035 & 0.061 & $42.2 \%$ \\
\hline Number of line crossings & 7.91 & 4.50 & $-75.7 \%$ \\
\hline \multicolumn{4}{|l|}{$\begin{array}{l}\text { Cone-field task: } \\
\text { working memory - }\end{array}$} \\
\hline general mean & 0.018 & 0.032 & $43.6 \%$ \\
\hline linear trend & 0.022 & 0.024 & $8.3 \%$ \\
\hline $\begin{array}{l}\text { reference memory - } \\
\text { general mean }\end{array}$ & 0.027 & 0.026 & -2.9 \\
\hline linear trend & 0.015 & 0.018 & $17.1 \%$ \\
\hline $\begin{array}{l}\text { Inhibitory avoidance: } \\
\text { step-through latency }\end{array}$ & 48.55 & 48.52 & $-0.1 \%$ \\
\hline $\begin{array}{l}\text { Progressive ratio: } \\
\text { food motivation }\end{array}$ & 14.30 & 11.35 & $-26.0 \%$ \\
\hline $\begin{array}{l}\text { Fixed-interval } 60 \mathrm{~s} \text { : } \\
\text { general mean }\end{array}$ & 0.038 & 0.020 & $-86.6 \%$ \\
\hline linear trend & 0.015 & 0.017 & $8.7 \%$ \\
\hline
\end{tabular}

Correlation between peak glucose level and non-cognitive behavioral parameters In young rats the peak glucose level was found to be negatively correlated with the time spent in the corner squares in the open-field test $\left(r_{p}=-0.60, p<0.05\right)$. The canonical correlation $\left(r_{c}\right)$ between the peak glucose level and both indices of emotionality in young rats was $0.67(F(2,14)=5.80, p<0.05)$. No other correlations between measures were observed $\left(r_{p}<0.39, n . s.\right)$. In old rats the peak glucose level was positively correlated with the total number of lines crossed in the open field test $\left(r_{p}=0.66, p<0.01\right)$. The canonical correlation $\left(r_{\mathfrak{c}}\right)$ between peak glucose level and both indices of emotionality in old rats was $0.66(\mathrm{~F}(2,15)=5.72, p<0.05)$. The peak glucose level was not correlated with other non-cognitive behavioral measures $\left(r_{p}<0.37\right.$, n.s. $)$. 
Correlation between choline acetyltransferase and learning parameters

In young rats hippocampal ChAT activity was found to be correlated with the linear decrease in escape latency in the Morris water-escape task $\left(r_{p}=-0.49, p<\right.$ 0.05 ), which suggests that the higher ChAT activity, the higher the rate of learning. No other correlations between ChAT activity and learning were observed in young rats $\left(r_{p}<0.35, n . s_{1}\right)$. There was no correlation between any learning parameter and ChAT activity in old rats $\left(r_{p}<0.19, n . s.\right)$.

Correlation between choline acetyltransferase and non-cognitive behavioral parameters

ChAT activity was not correlated with the behavioral parameters in young and old rats $\left(r_{p}<0.22, n . s.\right)$.

Age differences in variation in learning performance

Table III shows the standard errors of means and the percent difference for the behavioral parameters for young and old rats. There was no age-related difference in the percentual variation in behavior between the different tasks/tests (mean percentual difference $-2.2 ; t(11)=0.18, \mathrm{n} . \mathrm{s}$.).

\section{Discussion}

\section{Correlation between learning parameters}

The different learning parameters were not correlated in young and old rats, which indicates that learning performance in one task does not predict learning performance in another learning task. Moreover, the performance of the rats in the two tasks which were assumed to measure spatial discrimination learning (RM in cone-field task, and escape latency in Morris task) did not correlate. Markowska et al. (1989) also reported only a few interrelations between cognitive tasks in a study that evaluated individual differences in aged rats. They argued that the operationalizations of the measures and confounding variables could have attributed to this observation. In line with Markowska et al. (1989) we assume that the absence of a correlation between learning tasks can be attributed to the different operationalizations. For example, in the present study the two parameters of spatial discrimination learning were operationalized as the relation between visits to baited and unbaited cones (RM in cone-field task), and time to find the platform (escape latency in Morris task).

The lack of a correlation between the two spatial discrimination tasks supports our previous findings with young and old Lewis rats. We found that young rats adopt a habit of visiting unbaited cones in the cone-field task (van der Staay et al., 1990a) and that old Lewis rats do not use an allocentric orientation strategy in the Morris task (Blokland et al., submitted). Moreover, it has been reported that even similar operationalizations of spatial discrimination performance may not be generalizable over different tests (van Luijtelaar et al., 1989). The present study therefore underscores the notion that different cognitive processes may be involved in performance in different learning tasks (Markowska et al., 1989) and 
that these cognitive processes may decline independently during aging (van der Staay et al., 1990a).

The notion that other (non)cognitive processes may confound learning performance may have consequences for studies in which age-related differences in learning and memory are evaluated. Because it has been found that there are age-related differences in (non)cognitive processes (e.g., emotional reactivity, this study (see below); problem solving strategies, van der Staay et al., 1990a), learning performance may not be comparable across age groups. A within-subject design evaluates the relation between various parameters and reveals age-related differences with respect to these changes and does not compare the learning performance of young and old rats in one task.

The finding that there is no relation between different forms of learning and memory performance resembles findings from human studies (Witherspoon \& Moscovitch, 1989; Salthouse, 1990; Johnson \& Hasher, 1987) and implies that multiple cognitive processes are involved in performance in learning and memory tasks in humans and rats. Although this may be regarded as additional support for the use of animal models, the observation that different processes contribute to performance in learning and memory tasks may also entail problems. As long as the underlying processes which contribute to performance in learning and memory tasks are unknown in rats, one should be careful when interpolating data from animals to humans. It would be valuable to understand the (non)cognitive processes that contribute to the performance in learning and memory tasks and how these more distinct processes are affected by age.

It has been reported that old rats show a more heterogeneous performance than young rats (Gage et al., 1984b; Goodrick, 1972) (which was, however, found to be test dependent, see below). The'great variance in performance in old rats offers the possibility to make a distinction between 'poor' and 'good' performers (Goodrick, 1972; Gallagher \& Pelleymounter, 1988a). Studies in which old rats were divided into subgroups of "poor' and 'good' performers revealed that 'good' performers reached performance levels comparable to those of young animals whereas the old 'poor' performers showed a pronounced performance deficit (Goodrick, 1972). The distinction made on the basis of the behavioral performance was also reflected at a biochemical level. Brain glucose utilization (Gage et al., 1984b) and synaptic enhancement (Barnes, 1979) have been found to be correlated with spatial discrimination performance. However, in this study it was found that learning performance was not correlated between the different tasks. Therefore, a distinction between 'poor' and 'good' performers seems only to be valid for the task used and may not be generalized over tasks.

\section{Correlation among indices of emotionality}

As expected, the indices of emotionality were negatively correlated in young rats: the more emotional a rat reacts to a novel environment the more time it spends in the corner squares and the fewer lines it crosses during the OF test (van der Staay et al., 1990b). However, this relation was not found in the old rats, which indicates that novelty-induced emotionality is expressed differently in old 
rats in the open-field test. A similar change in the expression of emotionality with age has been found in a human study (Levenson et al., 1991). We have addressed the problems regarding the interpretation of the open-field data of old rats in an earlier study in which the effects of a nootropic drug were assessed (Blokland et al, 1990). The present results suggest that there is an age-related difference in emotional reactivity to a novel environment. It is conceivable that this a prioti difference in emotional reactivity could interfere with learning performance in learning tasks.

\section{Correlation between cognitive and non-cognitive behavior}

In the present study we were unable to find correlations between the cognitive and non-cognitive parameters of behavior. This suggests that the non-cognitive parameters of behavior in this study (emotional reactivity, motor performance, and food motivation) do not predict the learning performance of young and old rats in the learning tasks used in this study. However, this does not necessarily imply that these non-cognitive factors could not interfere with learning performance.

\section{Correlation between glucose and behavior}

In young rats the baseline glucose level was found to correlate negatively with the behavioral measure of food motivation. It could be argued that this correlation reflects a relation between the nutritional state and food motivation in young rats. Such a correlation was not observed in old rats suggesting that food motivation in old rats is also dependent on factors other than nutritional state. The lack of correlation in old rats could also reflect an age-related dysfunction of the system that regulates states of hunger/satiety.

There were no correlations between baseline glucose level and cognitive parameters in young rats. This lack of correlation in young rats could be due to an intact regulation of the blood glucose and that the blood glucose level of young subjects is within a range which has no behavioral consequences. This may explain why glucose regulation does not predict cognitive function in young rats (Stone et al., 1990) and why glucose administration has no effect on cognitive function in young human adults (Azari, 1991).

In old rats baseline glucose level and spatial discrimination learning (both WM and RM) in the cone-field task were negatively correlated. This suggests that the higher the baseline glucose level the poorer the spatial discrimination performance. Although this experiment can give no explanation for the relation between baseline glucose level and spatial discrimination learning in the conefield task, the data provide support for the hypothesis that blood glucose is related to cognitive functions in aging (Stone et al., 1988; Stone et al., 1990; Hall et al., 1989). It has been suggested that prolonged elevation of blood glucose levels may have neurotoxic effects (Craft et al, 1992). Our finding that higher a blood glucose level is associated with poorer performance in the cone-field task in old rats is consistent with this notion.

The other measure of blood glucose level was the peak level after injection, 
which was assumed to reflect the regulation of blood glucose: the higher the peak Jevel the poorer the regulation of blood glucose. It has been hypothesized that a poor blood glucose regulation is related to memory deficits in humans and animals (cf. Hall, 1989; Stone et al., 1990). We found a negative correlation between the peak level in blood and reference memory performance in the conefield task in old rats, which is in accordance with the hypothesis. However, we found that the peak level of glucose after injection was also correlated with the open-field test parameters in young and old rats. Although the open-field test is probably not a valid test in old rats (see above; Blokland et al., 1990), the correlation between peak glucose level and emotionality in young rats does suggest that the peak glucose level may also be related to emotionality. It is conceivable that the data of the study of Stone et al. (1990) and our study are affected by the methods of blood sampling. The procedure of glucose injection and blood collection, which is done by restraining a rat for a few minutes, results in a stress response, which has been found to increase blood glucose levels (de Boer et al., 1990). Thus, when assessing the regulation of blood glucose it would be preferable to use a method that minimizes stress during blood sampling. Although we found a relation between peak glucose level and reference memory in old rats, we cannot draw conclusions about the regulation of blood glucose and learning because it is possibile that the underlying process that influenced the peak glucose data is stress.

\section{Choline acetyltransferase and learning parameters}

The linear increase in spatial discrimination performance in the Morris task was correlated with hippocampal ChAT activity in young but not old rats. This suggests that the hippocampal cholinergic system is involved in spatial learning in the Morris task in young rats. Since this was the only correlation observed, we conclude that hippocampal ChAT activity does not predict learning performance. Moreover, the absence of a correlation between hippocampal ChAT activity and learning performance in old rats does not corroborate the notion that the agerelated decline in cognitive function is related to degenerative changes in the cholinergic system (Bartus et al., 1982) and a decrease in hippocampal function (Winocur, 1988). The poor correlation between hippocampal ChAT activity and learning performance in young and old rats is in accordance with the data of other studies (Luine \& Hearns, 1990; Markowska et al., 1989) and suggests that hippocampal ChAT activity, as a marker of the cholinergic transmission, does not predict learning performance in the old rat, i.e., in normal aging.

Age-related differences in variance in behavior

Our study does not provide support for the notion that old subjects show more variance in their behavior than young subjects (Goodrick, 1972; Gage et al., 1984b). There may be two explanations for this finding. 1) the results stem from two separate experiments, and 2) we selected the rats on particular criteria (no cataracts, no severe motor impairments, healthy state) to exclude possible interference with performance in the different tasks. This probably decreased the 
variance normally observed in a population of old rats. The possibility that the performance of the old group of rats is affected by pathologies and motor disturbances was minimized. The young rats showed a greater group variance on three different measures: activity in open-field, number of lever presses during test of food motivation, and general mean of fixed-interval $60 \mathrm{~s}$. This may indicate that the variance in young subjects is greater when the behavior is related to activity. Apparently, the group variance in behavior is not increased per se in old subjects but is related to task requirements.

Finally, three methodological aspects should be considered. The parameters of learning reflected performance during the acquisition phase, one trial block before the learning curves started to flatten, in the different tasks. The rationale for this approach was that the most dynamic changes occur during this phase. Furthermore, analysis of this part of the learning curve allows evaluation of two indices of learning: the rate of learning (slope of curve) and performance level (height of curve). The analysis used in this study took into account both indices of learning and may be more reliable than the evaluation of one index of learning (e.g., asymptotic level of performance; see also van Luijtelaar et al., 1989). Another methodological aspect that should be considered is that the animals were subjected to different tasks/tests. It has been found that test experience affects the performance of rats on subsequent learning tasks (van Luijtelaar et al., 1989). It cannot be ruled out that this could have affected the correlation between the different parameters. A last point to be mentioned is that the time between behavioral testing and assessment of biochemical parameters may affect the correlation between the parameters. The last two points should be considered as methodological limitations of this experimental approach.

In summary, the present study indicates that there are individual differences in learning and memory performance in different learning and memory tasks and that there are individual differences in cognitive aging. Apparently, learning performance is dependent on different (non)cognitive processes. Blood glucose level (baseline and peak level) seems to be correlated with different behaviors in old rats. Most interestingly, the correlation between baseline glucose levels and learning in old rats in the cone-field task was absent in young rat. The present results suggest that glucose regulation is affected during the process of aging and is correlated with cognitive performance. Finally, ChAT activity was only related with the rate of learning in the Morris task in young but not in old rats. This suggests that hippocampal ChAT activity does not predict learning and memory performance in normal aging. 


\title{
SPATTAL LEARNING DEFICIT AND REDUCED HIPPOCAMPAL ChAT ACTIVITY IN RATS AFTER AN I.CV. INJECTION OF STREPTOZOTOCIN
}

\author{
Arjan Blokland and Jellemer Jolles
}

\begin{abstract}
Intracerebroventricular (i.c.v.) injections of streptozotocin (STREP) lower the glucose utilization of the brain and affect the cholinergic system. The present study was designed to evaluate whether middle-aged STREP-treated rats have an impaired spatial discrimination performance in the Morris spatial navigation task. Performance in this task is sensitive to treatment with cholinergic antagonists. Spatial discrimination performance in the Morris task was impaired in the STREP-treated rats at the end of training. In STREP-treated rats, but not in control rats, spatial discrimination performance was associated with hippocampal choline acetyltransferase (ChAT) activity. The correlation between spatial discrimination performance in the Morris task and the decrease in hippocampal ChAT activity resembles the relation between cognitive and biochemical changes observed in Alzheimer's disease. Our findings suggest that STREP-treatment of middle-aged rats provides a relevant model for dementia.
\end{abstract}

\section{Introduction}

Recently, Hoyer and co-workers (Mayer et al., 1990; Nitsch et al., 1989; 1991) introduced a model in which brain glucose metabolism is decreased by an intracerebroventricular (i.c.v.) injection of streptozotocin (STREP). An i.c.v. injection of STREP impairs the brain insulin-insulin receptor system and consequently leads to a decrease in brain glucose utilization (Nitsch et al., 1991). It has been reported that a reduced brain glucose utilization is related to the agerelated decline in cognitive function (Gage et al., 1984b; Wree et al., 1991). A more dramatic decrease in glucose utilization has been observed in dementia (deLeon et al., 1983), suggesting that the severity of cognitive dysfunction is related to the level of brain glucose metabolism. So far, the effects of an i.c.v. STREP-injection on cognition have only been evaluated in inhibitory avoidance learning, where STREP-treated rats show an impaired performance, possibly as a result of a cholinergic dysfunction (Mayer et al, 1990). This explanation is supported by the finding that the level of choline acetyltransferase (ChAT) in the brain is reduced in STREP-treated rats (see Nitsch et al., 1991).

Spatial discrimination learning has consistently been found to decline with aging (Barnes et al., 1980; Rapp et al., 1987) and to be impaired after administration of cholinergic antagonists (Lindner Schallert, 1988; Whishaw, 1985). It can thus be hypothesized that i.c.v. STREP-treated rats will also show a deficit in spatial discrimination learning. It has recently been reported that spatial discrimination learning is impaired in patients with Alzheimer's disease which suggests that the cognitive deficits displayed by Alzheimer's patients might be an expression of an underlying abnormality in the hippocampus and the parietal cortex (Adelstein et al., 1992). Furthermore, the dedine in cognitive functions in 
Alzheimer's disease is related to a decrease in cholinergic markers (Katzman, 1986; Perry, 1980). Thus evaluation of the effects of STREP treatment on spatial discrimination learning and cholinergic activity could reveal whether the STREPtreated rat can be regarded as an animal model of dementia.

The aim of our study was to evaluate the effects of STREP on spatial discrimination learning in the Morris task. We also subjected the rats to an inhibitory avoidance task one week after training in the Morris task to evaluate whether we could reproduce the findings of Mayer et all. (1990). The behavioral data were correlated with hippocampal ChAT activity.

\section{Materials and methods}

\section{Animals}

We used sixteen 17 -month-old male Lewis rats, randomly assigned to the control group (CON; $n=8$ ) or the STREP group $(n=8)$. All rats were housed individually in standard Makrolon cages on sawdust bedding in an airconditioned room (about $20^{\circ} \mathrm{C}$ ). They were kept under a $12 / 12$ hour light-dark cycle (lights on from 9:00 to 21:00).

\section{Surgery}

One week before behavioral testing the rats were anesthesized with pentobarbital $(60 \mathrm{mg} / \mathrm{kg}$ i.p.) and placed in a stereotaxic frame. The STREP group was given a bilateral i.c.w. injection of STREP $(1.5 \mathrm{mg} / \mathrm{kg}$ body weight dissolved in $4 \mu \mathrm{l}$ saline, $2 \mu \mathrm{l}$ per injection site). The stereotaxic coordinates were: $-0.8 \mathrm{~mm}$ anterior, $1.5 \mathrm{~mm}$ lateral and -3.8 ventral from the bregma (Paxinos \& Watson, 1986). The CON group underwent the same surgical procedures but saline was injected instead of STREP. One STREP-treated rat died during training for unknown reasons.

\section{Procedures}

Ten days afler surgery, the rats were trained on the standard Morris spatial navigation task (Morris, 1981) in a black water-tank (diameter $1.22 \mathrm{~m}$ ). The rats were started from four different, randomly chosen, start positions and were trained to find an invisible platform (diameter $11 \mathrm{~cm}$ ) that was at a fixed position in the water tank, $1 \mathrm{~cm}$ below the surface of the water. The temperature of the water was $20-22^{\circ} \mathrm{C}$. A trial lasted until a rat had found the platform or until $60 \mathrm{~s}$ had elapsed. If a rat did not find the platform within $60 \mathrm{~s}$, it was placed on the platform for $3 \mathrm{~s}$ and then removed from the water tank. On the first day the rats were given 4 trials. The rats were given 8 trials on subsequent days to a total of 28 trials.

Four days after acquisition of the Morris task, the rats were subjected to the inhibitory avoidance task. The inhibitory avoidance apparatus consisted of a light and a dark compartment, each measuring $40 \times 25 \times 40 \mathrm{~cm}$. The light compartment was illuminated by a light bulb $(60 \mathrm{~W})$ mounted above $(40 \mathrm{~cm})$ the compartment. The floor consisted of a metal grid connected to a shock scrambler. The two 
compartments were separated by a guillotine door that could be raised $10 \mathrm{~cm}$. On day 1 the rats were placed in the light compartment and the latency to enter the dark compartment with four paws was measured. The guillotine door was lowered and a scrambled foot shock $(0.5 \mathrm{~s}, 1 \mathrm{~mA})$ was delivered. Twenty-four hours later, a retention trial was given. The latency to enter the dark compartment with four paws was measured.

\section{Choline acetyltransferase activity}

One week after training (three weeks after i.c.v. injection with STREP), the rats were decapitated and the heads were immediately immersed in liquid nitrogen for 8 seconds. The brains were taken out of the skull and the hippocampus was dissected at $0-4^{\circ} \mathrm{C}$. Tissue samples were stored at $-80^{\circ} \mathrm{C}$ until used to measure ChAT activity. Brain samples were homogenized $(5 \%)$ in $50 \mathrm{mM}$ phosphate buffer ( $\mathrm{pH} 7.4$ ) containing $0.2 \%$ Triton $\mathrm{X}-100$ and $20 \mathrm{mM}$ EDTA (pH 7.4). ChAT activity was determined according to the method of Fonnum (1975), using ${ }^{14 C}$ acetyl coenzyme-A (Amersham, $50-60 \mathrm{mCi} / \mathrm{mM}$ ).

\section{Statistical analysis}

The step-through latency in the 24-retention session minus the step-through latency during the first trial was taken as the parameter for inhibitory avoidance performance. Treatment effects were evaluated using a t-test. For analysis of the Morris task data the mean escape latency was calculated per block of four trials. Treatment effects were evaluated in a two-factorial (treatment and trial block) analysis of variance with repeated measures over trial blocks. Interdependencies of the different measures were analyzed using Pearson's correlation coefficient.

\section{Results}

STREP treatment did not affect the step-through latency during the first trial (step-through latency in $S$ (SEM): CON, $29.8(4.40)$; STREP, $27.0(8.60) ; t(13)=0.29, \mathrm{P}$ $>0.10)$ and there was no treatment effect on inhibitory avoidance learning (difference score in S (SEM); CON, $229.4(98.42)$; STREP, $158(87.31) ; t(13)=0.53, \mathrm{P}>$ $0.10)$.

Analysis of the data for the acquisition of the Morris task revealed that the rats reduced their escape latencies during training $(F(6,78)=14.07, \mathrm{p}<0.01$; see Fig. 1) and that the STREP-treated rats tended to have a poorer performance than the control rats (general mean: $F(1,13)=3.74,0.10>p>0.05$ ). At the end of training (last four trial blocks), the performance of the STREP-treated rats was found to be impaired (general mean: $F(1,13)=8.45, \mathrm{p}<0.05$ ).

Hippocampal ChAT activity (nmol/mg protein/hour) was decreased in the STREP-treated rats (mean (SEM); CON: $83.6(1.24)$, STREP: $45.0(12.27) ; t(13)=9.03$, $p<0.01$ ).

There was no correlation between inhibitory avoidance performance and hippocampal ChAT activity for either the CON or STREP group $(r=0.20$ and $r=$ 0.41 , respectively; for both correlations, $\mathrm{p}>0.10$ ). However, hippocampal ChAT 
activity correlated negatively with the mean escape latency of the STREP group during the last four trial blocks of training in the Morris task $(r=-0.86, p<0.05$; see Fig. 2). No correlation between the two measures was found for the CON group $(r=-0.22, n . s$.$) . Low hippocampal ChAT activity thus predicted poor$ swimming performance in the Morris task in the STREP-treated rats.

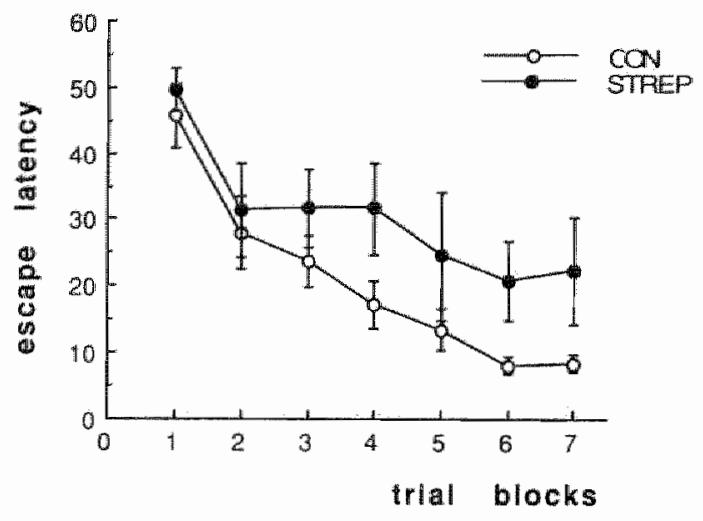

FIGURE 1: Escape latencies (in s \pm SEM) of 17 -month-old control (open cirdes) and streptozotocintreated (closed circles) Lewis rats during acquisition of the Morris spatial navigation task.

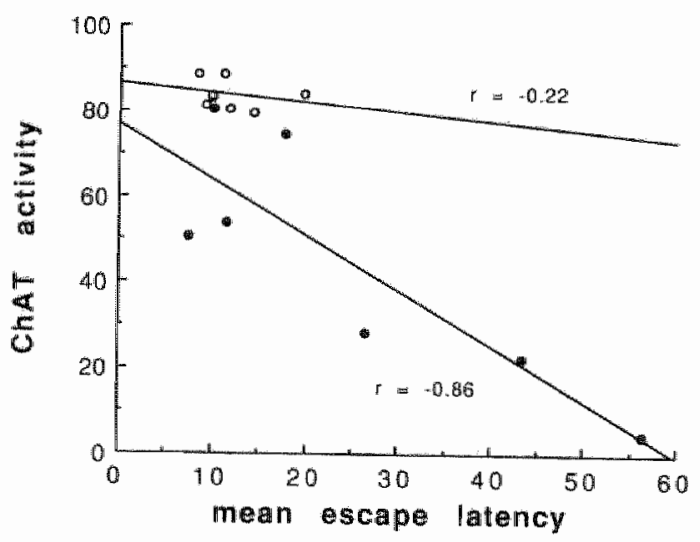

FIGURE 2: Relation between hippocampal choline acetyltransferase activity (rmol/mg protein/hour) and average escape latency during the last four trial blocks of training in the Morris task for 17 -month-old control (open circles) and streptozotocin-treated (closed circles) Lewis rats. 


\section{Discussion}

The present study showed that STREP treatment affected spatial discrimination performance in the Morris task at the end of training. This performance deficit of the STREP-treated rats was correlated to a decrease in hippocampal ChAT activity. Such a relation between spatial discrimination learning and the (hippocampal) cholinergic system has been found in other studies (e.g., Decker et al., 1988; Lindner \& Schallert, 1988).

In contrast to the study of Mayer et al. in which 12-month-old rats were used (Mayer et al., 1990), we did not observe a STREP-induced impairment in inhibitory avoidance learning. However, it should be mentioned that the mean difference score was higher for the CON rats than for the STREP rats but that the variation in both experimental groups was high. This large within group variation may be due to the age of the rats used in the present experiment (see also below). Another explanation for the different results could be that there were differences in the strain of rats used, the apparatus, and the parameters used in the inhibitory avoidance task.

We also evaluated the effects of an i.c.v. injection of STREP in 3-month-old rats but did not find a spatial discrimination performance deficit at the end of training (data not showm). We assume that young rats are able to reverse/compensate for the effects of STREP treatment. However, the young STREP-treated rats showed an impaired inhibitory avoidance performance, thereby corroborating the results of Mayer et al. (1990). Mayer et al. (1990) assumed that the shorter step-through latency during the test trial in the inhibitory avoidance task was not attributed to the STREP-induced increase in spontaneous locomotor activity because the step-through latency in the first trial did not differ for the STREP and control groups. It could, however, be argued that the STREPinduced increase in spontaneous locomotor activity might not be detected during the first trial because both the control and STREP groups had a short initial stepthrough latency. In the test trial the STREP-induced increase in spontaneous locomotor activity could have decreased the step-through latency. These data should therefore be interpreted with caution. The absence of a STREP-induced learning impairment in the Morris task in young rats underscores the possibility that inhibitory avoidance learning in young STREP-treated rats is affected by an increase in spontaneous locomotor activity. Because inhibitory avoidance learning was not affected by STREP treatment in middle-aged rats, it is unlikely that the impaired spatial discrimination performance of these rats was affected by a STREP-induced increase in spontaneous locomotor activity.

An i.c.v. injection of STREP not only affects the cholinergic system but also affects the concentration of different monoaminergic neurotransmitters (noradrenaline, dopamine and serotonin) in the rat brain differently (Ding et al., 1992). It could be argued that a change in these neurotransmitter systems could also have affected spatial discrimination performance in the Morris task. However, in the present study we found a strong correlation between escape performance and hippocampal ChAT activity, which suggests that there is a 
relation between the two measures. Such a correlation between cognitive function and ChAT activity has also been found in Alzheimer's disease (Katzman, 1986; Perry, 1980). In addition, it has been found that ${ }^{\text {a }}$ apart from the marked decrease in actetylcholine, Alzheimer's disease is accompanied by changes in different monoaminergic neurotransmitter systems (see Ding et al., 1992). Thus, although other neurotransmitter systems are affected in the STREPtreated rat and in Alzheimer's disease, there is a resemblance in the relation between the decline in cholinergic function and cognitive processes. This observation suggests that the middle-aged STREP-treated rat can be regarded as an animal model for the cognitive decline obseved in dementia. Furthermore, it has been suggested that the impairment of carbohydrate and related amino acid metabolism in Alzheimer's disease is related to an impairment of the insulininsulin receptor system, which is also affected by i.c.v. administered STREP (Nitsch et al., 1989). A similar dysfunction of an underlying fundamental biochemical process may explain the similarities between the biochemical and cognitive changes in Alzheimer's disease and in the middle-aged STREP-treated rat. The present results encourage further investigation of the effects of STREP at both a behavioral and a biochemical level. 


\title{
DIFFERENTIAL EFFECT OF ACETYL-L-CARNITINE ON OPEN FIELD BEHAVIOR IN YOUNG AND OLD RATS
}

\author{
Arjan Blokland, Wijnand Raaijmakers, F. Josef van der Staay \\ and Jellemer Jolles
}

\begin{abstract}
Acetyl-l-carnitine (ALCAR) exerts anti-aging effects with regard to various physiological and neurochemical measures in rats. In this study we evaluated the effect of a chronic treatment with ALCAR 6 weeks, 50 $\mathrm{mg} / \mathrm{kg} /$ day) on the open field behavior of 5 month-old and 26 -month-old male Wistar rats. Old rats defecated more, made fewer crossings, and spent more time in the corner squares. However, it is difficult to interpret these findings in terms of an increase in emotional reactivity with age because locomotor performance generally has been found to be impaired in old rats. Consequently, treatment effects were analysed within age groups. ALCAR had no effect in young rats, but the old ALCAR-treated rats ambulated less and spent hore time in the corner squares than the old control rats. We have interpreted this in terms of an enhanced emotional reactivity of old ALCAR-treatedrats.
\end{abstract}

\section{Introduction}

Various nootropic substances have been developed in the search for a potential pharmacological treatment of cognitive disturbances in elderly subjects. One of these substances is Acetyl-1-carnitine (ALCAR). ALCAR is the acetyl derivate of carnitine, a naturally occurring substance which plays an important role in the energy and lipid metabolism of cells (Curti et al., 1986; Drago et al., 1986). As ALCAR cancross the blood-brain barrier, it could affect brain cells directly (see Angelucci et al., 1986).

Chronic ALCAR treatment is reported to exert anti-aging effects as it prevents several age-associated neuroanatomical and neuro-chemical changes, particularly in the hippocampus (Badiali de Georgi et al., 1987; Curti et al., 1986). Another study has shown that the control of adrenocortical responsiveness is improved in aged ALCAR-treated rats (Angelucci et al., 1986). This finding is consistent with the anatomical and functional preservation of hippocampal glucocorticoid receptors found in old rats chronically treated with ALCAR (Angelucci et al., 1986). Furthermore, chronic ALCAR treatment has been shown to improve discrimination learning in 19-month-old rats compared to untreated controls (Ghirardi et al., 1988).

ALCAR treatment increases the levels of carnitine and ALCAR inthe brain. I has been suggested. that basal forebrain cholinergic neurons are activated by the increase in high-affinity choline uptake activity in cortical and hippocampal but not striatal synaptosomes (Curti et al., 1986).

The effects of ALCAR on basal aspects of behavior are not clear. Drago and co-workers (1986) found that young rats showed more ambulation and reared 
more frequently in an open field (OF) after acute injections of ALCAR (1 $\mathrm{mg} / \mathrm{kg}$, i.p.) than young untreated rats. Increased ambulation is commonly interpreted as reflecting a lower level of emotionality (Broadhurst, 1957; van der Staay et al, 1990b). We decided to study the effects of chronic ALCAR treatment on open field behavior. Besides increased ambulation (number of squares crossed) (Hall, 1936), fewer boli (Goma \& Tobena, 1978) and a decreased occupancy of comer squares (van der Staay et all, 1990b) were taken to indicate lower 'emotional reactivity'. An increased" emotional reactivity", on the other hand, should express itself in changes in these measures in the opposite direction.

Both young and old rats were used in the present study. In view of the presumed nootropic activity of ALCAR it is of interest to study the effects of chronic ALCAR on OF behavior in old rats. An age-associated decrease in locomotor activity (Willig et al., 1987) may interfere with the interpretation of the expected lower ambulation of old rats in the OF. Schuurman and Traber (1989) reported a decrease in ambulation of about 30 per cent between young (3-4 months) and aged (21-25 months) rats of the same strain used in this study. Since there are no relevant validation studies, it is not certain whether an age-related decline of $O F$ ambulation represents a fall in emotional reactivity, in arousal (Shuurman \& Traber, 1989), or in motor skills (Gage et al., 1984a). We assume, however, that the OF measures are valid to detect ALCAR-induced emotional changes within age groups, which is the main objective of the present study. Furthermore, we hypothesized that aged rats would be less active than young rats as reported by others (Gage et al., 1984a; Shuurman \& Traber, 1989; Willig et al., 1987). We compared the untreated age groups to test this hypothesis.

\section{Materials and methods}

\section{Animals}

We used male random-bred Wistar rats (Bor:WISW(SPFCpb), Winkelmann, Borchen, West Germany) from two shipments, each consisting of eight young (2-month-old) and eight old (23-month-old) animals. Rats from each shipment were matched for body weight and assigned to one of four groups: young untreated $(n=4)$, young ALCAR-treated $(n=4)$; old untreated $(\mathrm{n}=4)$, and old ALCAR-treated $(\mathrm{n}=4)$. All animals were housed individually in standard Makrolon cages and had free access to food and water. The rats were kept under a reversed light-dark cycle (lights on between 21:00 and 9:00).

\section{Treatment}

Six weeks after the animals had been matched, the ALCAR-treated groups were given $50 \mathrm{mg} / \mathrm{kg} /$ day ALCAR, which was dissolved in a $0.1 \%$ solution of saccharine. The control groups received a comparable amount of saccharine solution $(10 \mathrm{ml} 0.1 \%$ per day). Four weeks after the treatment started, the rats were handled extensively on four successive days. Two weeks later the 
animals were tested in the OF.

Behavioral testing was carried out between 10:00 and 13:00. The drinking bottles containing the solutions were offered two hours before the OF test was performed. All animals consumed the total amount of the sweetened solutions within a few minutes. A drinking bottle containing tap water was always present.

\section{Procedures}

The OF consisted of a square base $(100 \times 100 \mathrm{~cm})$ subdivided into 36 equal squares by black lines; the walls were $30 \mathrm{~cm}$ high. The floor was made of white Trespa $^{\mathrm{TM}}$ and the four walls were made of transparent Plexiglass ${ }^{\mathrm{TM}}$. Two red flluorescent tubes provided very dim illumination (about two lux) on the floor of the apparatus.

Immediately after a rat was placed in the centre of the OF the movements of the rat were scored. A crossing was scored as soon as the rat crossed line with both hind legs. After each session the number of boli was counted. "The number of lines crossed and the total time spent in the corner squares were recorded manually with an AIM 64-micro computer. The experimenter sat in front of the OF. Testing was carried out on four consecutive days in five-minute sessions.

\section{Statistical analysis}

In order to enhance reliability, the data from the four sessions were aggregated as proposed by Ossenkopp and Mazmanian (1985). Three measures of $O F$ behavior were subjected to statistical analysis:

- Mean number of boli per day (the mean number of boll were transformed to rank scores because of the frequent occurrence of zero scores, predominantly in young rats).

- The mean daily number of crossing of all squares.

- The mean time $(t)$ in seconds per session spent in the corner squares in seconds transformed to the natural logarithm $\mathrm{Ln}((\mathrm{t}+12+13+14) / 4)$ (subscripts refer to day 1 through 4 of testing) in order to remove inhomogeneity.

Shipment effects were analysed in an age by treatment by shipment analysis of variance (ANOVA). The behavioral measures of animals of both shipments were the same (all Fs involving main effect and interactions with shipment were not significant). Thus the second shipment can be considered as a replication of the first one. The data for both shipments were therefore pooled.

As mentioned in the Introduction, age-related changes were analysed by comparing the untreated groups with t-tests. The effects of the chronic ALCAR treatment were assessed within age groups by using t-tests. Age differences and treatment effects on the number of boli were analysed by Kruskal-Wallis. one-way analysis of variance by ranks (Chi2-approximation). 


\section{Results and discussion}

The treatment and control groups weighed the same after the data for both shipments had been pooled. The mean body weight (grams \pm SEM) was 288.1 ( \pm 3.6$)$ for the untreated young rats, $290.5( \pm 4.5)$ for the ALCAR-treated young rats, 499.4 ( \pm 11.1 ) for the untreated old rats, and $515.7( \pm 9.8)$ for the ALCAR-treated aged rats. At testing, 11 weeks after matching the body weights were $379.6( \pm 10.4), 375.9( \pm 9.1), 507.1( \pm 12.3)$, and 515.0 ( \pm 17.5$)$ grams, respectively. Thus, the young rats had gained weight; there was no indication that the ALCAR treatment affected the body weight. The weights of the aged rats were stable over the entire period from matching to testing.

The results of OF behavior are summarized in Table I. Compared with the young untreated rats the old untreated rats made fewer crossings $(t(14)=4.1, p$ $<0.01)$, spent more time in the corner squares $(t(14)=-2.7 p<0.05)$, and defecated more (Chi2 $=9.2$, df $=1, p<0.01$ ). Although it is tempting to conclude that aged rats react more emotionally than young rats when confronted with a novel environment, no attempt was made to interpret age differences in OF behavior because of the lack of data on the validity of these measures across ages. The decrease in ambulation in the old rats is quite similar to the value (ca 30\%) reported by others for rats of the same strain (Schuurman \& Traber, 1989).

Chronic treatment with ALCAR had no effect on the OF behavior of young rats. This result contrasts with the findings reported by Drago and co-workers (1986). The results of the two studies, however, cannot be compared directly because of procedural differences. Drago et al, used acute injections $(1 \mathrm{mg} / \mathrm{kg}$, i.p.) while we used a chronic oral administration of ALCAR. Another procedural difference is that Drago and co-workers tested the rats using the OF procedure described by Weijnen and Slangen (1970). In this procedure rats are tested for one day in a brightly lit circular arena. In our study rats were tested in a dimly lit square OF on four consecutive days, in order to enhance the reliability of the OF measures (Ossenkop \& Mazmanian, 1985; van der Staay et al., 1990b).

Old ALCAR-treated rats made fewer crossings $(t(14)=2.30, p<0.05)$ and spent more time in the corner squares $(t(14)=-2.71, \mathrm{p}<0.05)$ than the old untreated animals. This suggests that the treatment increased the emotional reactivity of the old rats when they were exposed to a novel environment. No treatment effect, however, was found for the number of boli. An alternative explanation in terms of a change in peripheral motor function cannot be excluded but is highly unlikely. If any, the expected effect of the ALCAR treatment would be to increase ambulation in the old rats as a result of the stimulatory effects of ALCAR on the energy and lipid metabolism of muscle cells (see Angelucci et al., 1986; Curti et al., 1986). A similar argument holds with regard to a possible increase in arousal or excitability. According to Onofrj et al. (1983), ALCAR-treated rats show heightened arousal. An increase in ambulation would then be expected rather than the decrease observed. 
TABLE I: Mean number ( \pm SEM) of boli (between parentheses: median), mean number of crossings of all squares and the average time spent in comer squares (in s and transformed to the natural logerithm) of untreated and ALCAR-treated young ( 5 months) and aged ( 26 months) male Wistar rats ( $\mathrm{n}=$ 8 per groupl.

*: treatment effect; $p<0.05$.

\begin{tabular}{|c|c|c|}
\hline up & $\begin{array}{l}\text { number } \\
\text { of boli }\end{array}$ & $\begin{array}{l}\text { number of } \\
\text { squares crossed }\end{array}$ \\
\hline
\end{tabular}

5-month old untreated $0.06 \pm 0.06 \quad 55.93 \pm 2.81 \quad 4.75 \pm 0.05$ (0.0)

$\begin{array}{lccc}\text { ALCAR- } & 0.28 \pm 0.20 & 54.55 \pm 3.92 & 4.75 \pm 0.07 \\ \text { treatted } & (0.0) & & \end{array}$

$$
\text { Chi2 }=-1.06 \quad t=0.76 \quad t=-0.03
$$

26-month-old untreated

ALCARtreated $3.19 \pm 1.03$ (2.9)

Chi2 $=-0.31$

$$
2.81 \pm 0.63
$$

$+$

In a recent studly we assessed the validity of the number of crossings and time spent in the corner squares of an OF as measures of emotional reactivity (van der Staay et al., 1990b). Young-adult rats of seven inbred strains were observed in the OF and a light-dark preference box. Both tests are supposed to measure emotional reactivity. Analysis by standard cross-validation techniques and by genetic correlations in a multitrait-multimethod-like design revealed that the above-mentioned $\mathrm{OF}$ measures are valid measures of emotionality in the rat. This suggests that chronic treatment with ALCAR enhances the level of emotionality in aged rats. Such a notion does not readily fit into the supposed action of ALCAR on the responsiveness of the corticosterone system.

There are few studies on the influence of aging on emotionality (Elias \& Elias, 1977). Old rats are less capable of terminating an adrenocortical stress response (Sapolsky et al., 1983), but this has not been related to OF behavior as far as we know.

The possibility that emotionality is expressed differently in young and old rats is supported by the finding that diazepam does not have the usual anti-conflict effect in the Geller-Seifter test when applied to old rats 
(Kominsky et al., 1987). Such findings underline the necessity to assess the validity of the measures of emotionality in senescent rats, a study which we are currently carrying out. 


\title{
NEUROPROTECTIVE EFFECTS OF ACETYL-L-CARNITINE IN A NEW PARADIGM FOR BEHAVIOURAL ASSESSMENT DURING ACUTE METABOLIC INHIBITION
}

\author{
Arjan Blokland, Wiel Honig and Jelle Jolles
}

\begin{abstract}
In the present study the effects of acelyl-1-camitine (ALCAR) were evaluated in a new method to assess the behavioural effects of a brief period of hypoxia in the brain. In this method, cyanide (NaCN) is injected into the lateral ventricles and a well-learnt behaviour (spatial navigation) is measured shortly thereafter. In young rats, spatial navigation performance was found to be impaired 1 and 5 minutes after an i.c.v. injection $5.0 \mu \mathrm{g} \mathrm{NaCN}$ but not after $2.5 \mu \mathrm{g}$ NaCN. The performance of the old rats was not affected after both doses of NaCN. Chronic treatment with ALCAR attenuated the NaCN-induced (dose 5.0 \#g) behavioural deficit in young but not in old rats. We hypothesize that chronic treatment with ALCAR enthances the avallability and use of alternative energy sources during metabolic inhibition and may preserve neuronal function during these periods.
\end{abstract}

\section{Introduction}

Acetyl-1-carnitine (ALCAR), a nootropic drug, has been reported to alleviate cognitive function in aged rats (Caprioli et al., 1991; Ghirardi et al., 1988; Ghirardi et al., in press). In two studies in which the effects of chronic treatment with ALCAR were evaluated in various (non)cognitive tests, it was concluded that ALCAR did not affect sensorimotor skills and that ALCAR is probably involved in the long-term storage of information (Barnes et al., 1990; Markowska et al., 1990). Biochemically, acetyl-l-carnitine acts as a carrier of fatty acids across the inner mitochondrial membrane for subsequent $\mathbb{B}$-oxidation (Fritz, 1963). Further studies have revealed that ALCAR enhances the utilization of alternative energy sources (Aureli et al., 1990), which may reverse the age-related modifications in neuronal membranes (Schoeder, 1984). Gibson and Peterson (1981) reported that the age-related decrease in glucose utilization may cause a decrease in oxidative metabollism and the release and synthesis of acetylcholine. Since ALCAR enhances the utilization of alternative energy sources, ALCAR may compensate for the decrease in glucose utilization that occurs during aging and consequently may preserve neuronal function. Such a hypothesis can be tested in an animal model in which the energy status of the brain is lowered experimentally.

There are many substances and methods which are capable of influencing brain function by changing biochemical processes involved in energy metabolism in the brain (Auer et al., 1984; Baughman et al., 1990; Crane et al., 1978; de Ryck, 1990; Hoyer \& Krier, 1986; MacMillan, 1989). Models in which the energy status of the brain is affected are used in studies to evaluate drugs which are thought to have neuroprotective properties. Such drugs reverse the development of abnormalities and/or behavioural deficits after a period of metabolic inhibition. 
However, these models do not allow assessment of behaviour during the period of metabolic inhibition. For instance, Gibson and co-workers (Gibson et al., 1983) evaluated the behavioural effects of nitrite-induced hypoxia. The interpretation of the behavioural data, however, was complicated by the peripheral effects of nitrite, for instance, a decrease in motor function (Behroozi et al., 1972). The acute effects of a period of anoxia/hypoxia can only be assessed in biochemical or pathological studies which evaluate biochemical markers or neuronal damage, respectively (Auer, 1984; Peruche et al., 1990; Pörtner et al., 1990).

We developed a method in which behaviour can be assessed during a period of acute metabolic inhibition. Cyanide $(\mathrm{NaCN})$ is injected directly into the lateral ventricles of rats and a well-learnt behaviour is measured during the period that cyanide has its effect on biochemical processes in the brain. NaCN blocks the use of $\mathrm{O}_{2}$ in the mitochondria and consequently induces hypoxia (i.e., histotoxic hypoxia; MacMillan, 1989). We administered $\mathrm{NaCN}$ by this route to avoid peripheral effects. Moreover, $\mathrm{NaCN}$ per se does not cause neuronal damage (Brierly et al., 1976). Furthermore, low doses of cyanide are converted by mitochondrial sulphur transferase into non-toxic sulphur cyanide within minutes (Hathway, 1984). Finally, we injected the $\mathrm{NaCN}$ into the lateral ventricles because the $\mathrm{NaCN}$ would probably affect hippocampal function, which is known to be critically involved in the performance in the Morris task (Morris et al., 1982).

In this study we evaluated the hypothesis that ALCAR can alleviate the behavioural deficit observed during metabolic inhibition in young and old rats. We hypothesized that ALCAR preserves neuronal function and, thus, behavioural performance during a period of hypoxia by increasing the availability by alternative sources of energy.

\section{Materials and methods}

\section{Animals}

We used male Lewis rats from two shipments. The first shipment consisted of fifteen 3 -month-old rats which were divided randomly into two groups (CON young, $n=7$; ALCAR young, $n=8$ ). The second shipment consisted of twelve 3 month-old and sixteen 22-month-old rats (CON young, $\mathrm{n}=6$; ALCAR young, $\mathrm{n}=$ 6; $\mathrm{CON}$ old, $\mathrm{n}=8 ;$ ALCAR old, $\mathrm{n}=8$ ). All rats were housed individually in standard Makrolon cages on sawdust bedding in an air-conditioned room (about $20^{\circ} \mathrm{C}$ ). They were kept under a $12 / 12$ hour light dark cycle (lights on from 9:00 to 21:00). During the experiment one old CON rat died for unknown reasons.

\section{Treatiment}

ALCAR treatment was started two weeks before the rats acquired the Morris


drug was dissolved in a $0.1 \%$ solution of saccharine. The young and old CON group received a comparable amount (10 and $20 \mathrm{ml}_{\text {, }}$ respectively) of saccharine solution. One week after start of ALCAR treatment, the rats were anaesthetized 
with pentobarbital (60 $\mathrm{mg} / \mathrm{kg}$ i.p.), and bilateral stainless steel cannulas (outer and inner diameter 0.65 and $0.30 \mathrm{~mm}$, respectively) were implanted above the lateral ventricles. The cannulas were fixed to the skull with acrylic dental cement (Paladur). The coordinates for injection sides were: $-0.08 \mathrm{~mm}$ anterior, $1.5 \mathrm{~mm}$ lateral and $-3.8 \mathrm{~mm}$ ventral from the bregma (Paxinos \& Watson, 1986).

\section{Procedures}

Spatial discrimination learming: One week after surgery the rats were trained on the standard Morris spatial navigation task (Morris, 1981) in a black watertank (diameter of $1.22 \mathrm{~m}$ ). The rats were started from four different, randomly chosen, start positions and trained to find an invisible platform (diameter $11 \mathrm{~cm}$ ) that was at a fixed position in the water tank, $1 \mathrm{~cm}$ below the surface of the water. The temperature of the water was $20-22^{\circ} \mathrm{C}$. A trial lasted until a rat had found the platform or until $60 \mathrm{~s}$ had elapsed. If a rat did not find the platform within $60 \mathrm{~s}$ it was placed on the platform for three seconds, and then removed from the water tank. On the first day the rats were given four trials. The rats were given eight trials on consecutive days until the rats had reached asymptotic performance.

Effects of $\mathrm{NaCN}$ injections: After the rats had reached asymptotic performance, the effects of $\mathrm{NaCN}$ injections were evaluated. On day one the rats received four trials, and were then injected with $\mathrm{NaCN}(2.5 \mu \mathrm{g} / 2.5 \mu \mathrm{l} /$ injection site). The rats were given four additional trials 1, 5, 10 and 15 minutes after the $\mathrm{NaCN}$ injection. The four possible start positions were balanced per group during each post-injection trial. On day three the rats were subjected to the same procedure as on day one, but now a dose of $5.0 \mu \mathrm{g} \mathrm{NaCN} / 2.5 \mu \mathrm{l} /$ injection site was administered. The same procedure with both doses was repeated one week later.

\section{Statistical analysis}

Shipment effects: Shipment effects for young subjects were analysed in a twofactorial analysis of variance (shipment and group). The behavioural measures of animals from both shipments were the same (for acquisition training and $\mathrm{NaCN}$ sessions: all $\mathrm{p}$ values $>0.10$ for $\mathrm{F}$ values involving main effect and interactions with shipment). Thus the second shipment can be considered as a duplicate of the first one. The data from both shipments were therefore pooled.

Spatial discrimination learning: Escape latencies were analysed per trial block of 4 trials. Differences in escape performance over trial blocks were analysed in a three-factorial (age, treatment and trial block) analysis of variance with repeated measures over trial blocks. In addition, differences in learning curves were analysed by comparing the individual orthogonal trend components calculated over the first four trial blocks, which correspond to the first phase of acquisition. The asymptotic performance of the old CON and ALCAR-treated rats was evauated by using $\mathrm{t}$-statistics.

Effects of $\mathrm{NaCN}$ injections: The mean escape latencies of the pre-injection trials were averaged and taken as baseline performance for each rat. In order to evaluate whether $\mathrm{NaCN}$ treatment affected the performance of the rats, the escape latency during the trials after the animals had received $\mathrm{NaCN}$ was 
compared with baseline performance by using $\mathrm{t}$-statistics. The mean escape latency of the two sessions 1,5 and $10 \mathrm{~min}$ after injection was calculated for each dose of $\mathrm{NaCN}$. For each dose of $\mathrm{NaCN}$, ALCAR treatment effects on escape latency 1,5 and $10 \mathrm{~min}$ after $\mathrm{NaCN}$ injections were analysed for each age group separately in a two-factorial (treatment and minutes) analysis of variance with repeated measures over minutes. The age groups were analysed separately because of the different mechanisms that may underlie performance in the Morris task (see Discussion).

The performance of one young control rat from the first shipment was not affected after $\mathrm{NaCN}$ injections, and this rat was excluded from the statistical analysis. The lack of effect is suggestive of an incorrect cannula placement. Unfortunately, however, the canula placement could not be verified because the brains were used for a biochemical study.

\section{Results}

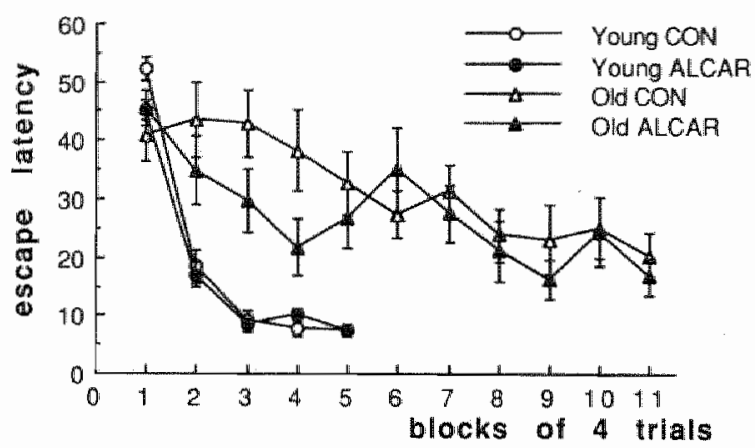

FIGURE I: EScape latency (in s \pm SEM) of young and old, control (CON) and ALCAR-treated, rats during acquisition of the Morris water escape task.

Spatial discrimination learning: There was an age-related performance deficit in the acquisition of the Morris task (general mean: $F(1,37)=50.89, p<0.01$ ). Chronic treatment with ALCAR improved performance in the Morris task during the first phase of training (general mean: $F(1,37)=5.0, p<0.05$; see Fig. 1). Further analysis revealed that ALCAR enhanced the performance of the old rats but had no effect in the young rats during the first phase of training (age by treatment by block interaction: $F(3,111)=5.60, p<0.01)$. The increase in performance was characterized by a linear trend $(F(1,37)=229.08, \mathrm{p}<0.01$ ) which explained $87 \%$ of the variation. The old ALCAR-treated rats reduced their escape 
latency faster than the old control rats (age by treatment by linear trend interaction: $F(1,37)=18.65, p<0.01$ ). At the end of training, the performance of the old CON and old ALCAR-treated rats no longer differed (last four trial blocks: ts $<1.1$, ns), indicating that the old CON and ALCAR animals had reached a similar asymptotic level of performance.

Effects of NaCN injections: During the four trials before the injection, the young and old ALCAR-treated rats never differed from their respective control groups ( $\mathrm{ts}<1.7, \mathrm{~ns})$.

Dose $2.5 \mu \mathrm{g}$. After injection with a dose of $2.5 \mu \mathrm{g}$, the performance of the young rats was not affected significantly (ts $<1.9$, ns) and did not change during subsequent trials (minutes effect: $\mathrm{F}(2,48)<1$, ns, see Fig. 2 left panel). There was only weak statistical evidence that the performance of the young CON deviated from baseline performance during the first trial, i.e. 1 minute after injection (t(11) $=1.94,0.10>\mathrm{p}>0.05$ ). The escape latency of the old rats did not deviate from baseline performance (ts $<1.3$, ns; see Fig. 2 right panel) and did not change during post-injection trials (minutes efect: $\mathrm{F}(2,26)<1$, ns).
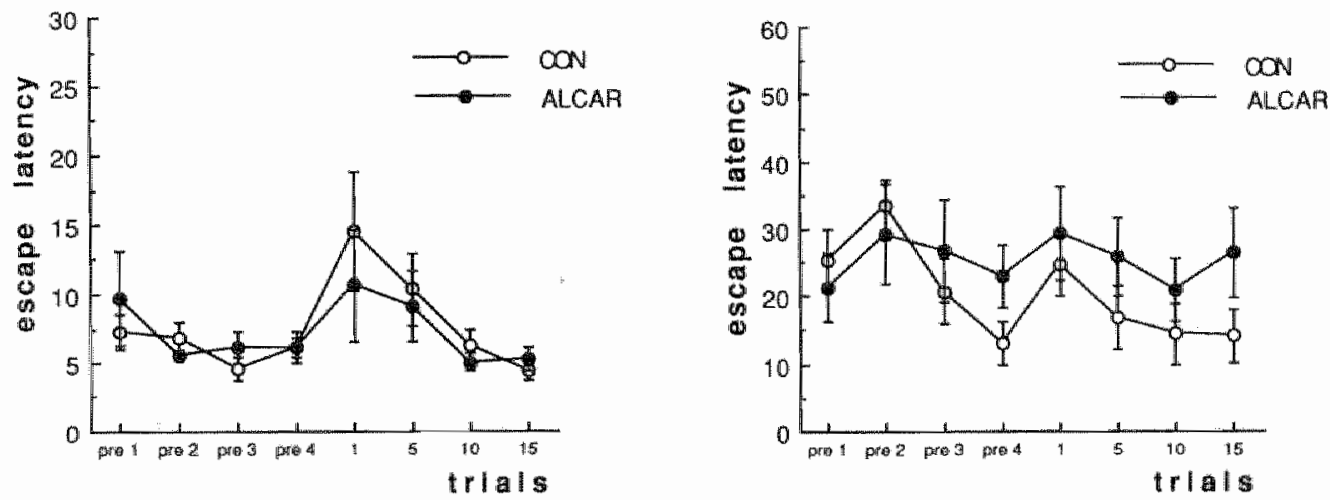

FIGURE 2: Escape latency (in $\$ \pm$ SEM) of young (left panel) and old (right panel), CON and ALCARtreated, rats during the four pre- and post-injection $(\mathrm{NaCN}, 2.5 \mu \mathrm{g})$ trials $(1,5,10$, and 15 minutes after injection) in the Morris water escape task.

Dose $5.0 \mu \mathrm{g}$ : After a dose of $5.0 \mu \mathrm{g}$, the escape latency of the young CON and ALCAR-treated rats deviated from baseline performance during the first two trials after injection, i.e. 1 and 5 minutes after $\mathrm{NaCN}$ injection ( $\mathrm{t}$ values $>2.27, \mathrm{p}$ values < 0.05; see Fig. 3 left panel). During the first trial, 1 minute after injection, the increase in escape latency of the young ALCAR rats was less than that of the young $C O N$ rats $(t(24)=2.16, p<0.05)$. The escape latency decreased during the first three trials after injection (minutes effect: $F(2,48)=8.09, p<0.01$ ). The escape latency of the young CON rats during these trials was higher than the escape 
latency of the young ALCAR animals (general mean: $(F(1,24)=5.34, p<0.05)$. The performance of the old CON and ALCAR animals did not deviate from baseline performance after $\mathrm{NaCN}$ injection (t values < 1.4, ns; see Fig. 3 right panel). Furthermore, the performance of the old CON and ALCAR animals did not differ after the $\mathrm{NaCN}$ injections ( $t$ values $<1.3, \mathrm{~ns}$ ).
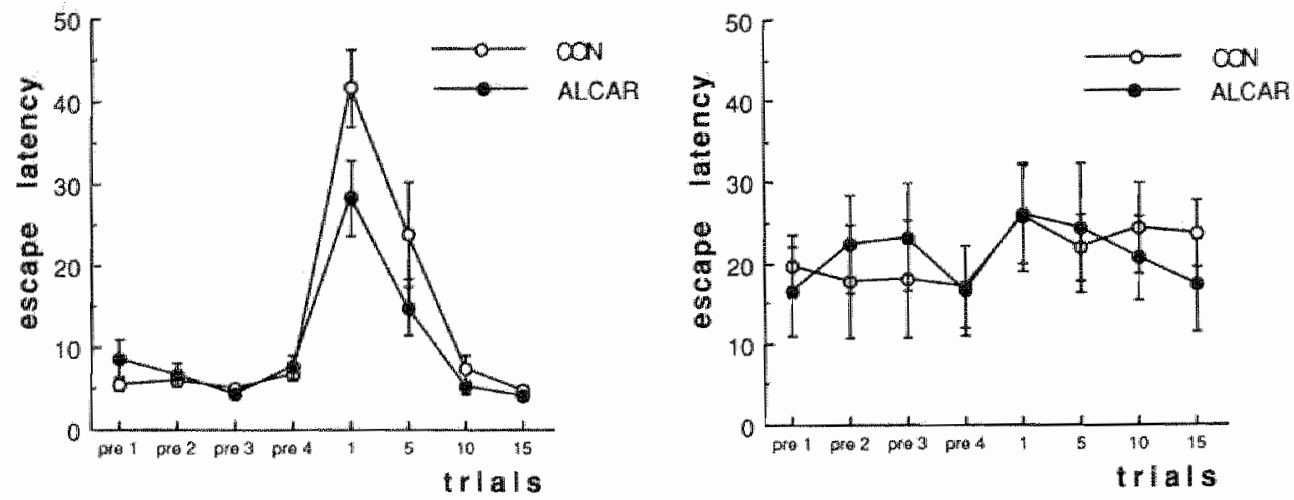

FIGURE 3: Escape latency (in s \pm SEM) of young (left panel) and old (right panel), CON and ALCARtreated, rats during the four pre- and post-injection $(\mathrm{NaCN}, 5.0 \mu \mathrm{g})$ trials $(1,5,10$, and 15 minutes after injection' in the Morris water escape task.

\section{Discussion}

Chronic treatment with ALCAR increased the rate of spatial discrimination learning of old rats during the first phase of training, which corroborates the findings of Ghirardi et al. (in press). In contrast, we found that ALCAR had no effect on escape peformance in old rats at the end of training. It has been reported recently that there is an age-related change in the use of problem-solving strategies in rats and that ALCAR antagonizes the age-related deterioration to learn and solve a radial maze task (Caprioli et al., 1991). However, ALCAR treatment did not affect the problem-solving strategy in a radial maze. In a separate study we found that young and old Lewis rats use different problemsolving strategies in the Morris task (Blokland et al., submitted). We assume that ALCAR improves the rate of learning in old rats but that the old ALCAR-treated rats used the same problem-solving strategy as the old CON rats in the Morris task. The lack of effect of ALCAR treatment during acquisition training in young rats is probably due to the fact that young rats reach asymptotic performance within only twelve trials, i.e., three trial blocks. It can thus be assumed that ALCAR had no effect in young rats because of floor effects.

When the energy state of the brain was lowered by an i.c.v. injection of $2.5 \mu \mathrm{g}$ $\mathrm{NaCN}$, the performance of the rats was not consistently affected. It can be argued 
that the rats were either able to compensate for the energy disturbance by using other energy sources or that this dose was too low to affect the energy state of the brain. However, a dose of $5.0 \mu \mathrm{g} \mathrm{NaCN}$ disrupted the performance of the rats 1 and 5 minutes after injection. It has been reported that if sulphur transferase is present in adequate amounts, cyanide is detoxified in approximately 15 minutes (Hathway, 1984). The duration of the behavioural effects of $\mathrm{NaCN}$ in the present study is thus comparable to the duration of the metabolic breakdown of the substance. Furthermore, we have measured lipid phosphorylation (an energydependent process) after an i.c.v. injection of $\mathrm{NaCN}$ (in preparation) and found a similar time course for the effects of $\mathrm{NaCN}$ on this measure.

Unexpectedly, $\mathrm{NaCN}$ treatment did not affect the performance of the old rats. It is conceivable that the absence of $\mathrm{NaCN}$ effects in the old rats could be due to a systematic error in the cannula placement in old rats. As mentioned in the methods section, we were not able to verify the cannula placement. Another explanation could be that the escape latency of the old rats was about twice as high as that of young rats and the variation was substantially greater. Moreover, old Lewis rats do not use an allocentric orientation strategy in the Morris task but probably swim in an arbitrary manner through the water tank (Blokland et al., submitted). It could be argued that $\mathrm{NaCN}$ did not affect this swimming behaviour in the old rats (see also below) which may explain the absence of $\mathrm{NaCN}$ and ALCAR treatment effects in the old rats.

In a study in which the protective effect of p-aminopropiophenone (a drug that binds to haemoglobin) after a systemic injection of cyanide was evaluated in a swimming task in guinea-pigs, it was suggested that cyanide could have affected cognitive function ( $\mathrm{D}^{\prime} \mathrm{Mello}$, 1986). This is supported by the finding that the brain is the primary target for cyanide (Way, 1984). In the present experiment we injected $\mathrm{NaCN}$ directly into the ventricles. It is well known that the hippocampus is a target structure for drugs that are injected i.c.v. Since disruption of hippocampal function is known to result in impairments in the Morris task (Morris et al., 1982; 1990), we tentatively conclude that $\mathrm{NaCN}$ caused a navigational impairment.

Chronic treatment with ALCAR was found to attenuate the $\mathrm{NaCN}$-induced (dose $5.0 \mu \mathrm{g}$ ) behavioural deficit in young rats. This finding was replicated in the present study and indicates that chronic treatment with ALCAR is able to preserve neuronal function during a period of metabolic inhibition. This is in accordance with our hypothesis that ALCAR enhances the use of alternative sources of energy during a period of metabolic inhibition. Furthermore, the present results could be related to the protective effects of 1 -carnitine in ammonia intoxication (Ohtsuka \& Griffith, 1991). In this study, Ohtsuka and Griffith assumed that 1 -carnitine sustained the energy needs during ammonia intoxication, which causes an energy deficit in the brain (Cooper \& Plum, 1988). The additional value of the model used in the present study is that the biochemical mechanism of action of the nootropic drug ALCAR could be evaluated at a behavioural level. Until now, the behavioural and biochemical effects of ALCAR have been evaluated separately, and only speculative 
explanations could be given for the behavioural effects of ALCAR. From the results of the present study it can be hypothesized that the mechanism of action of the nootropic drug ALCAR could be related to an increase in availability of alternative energy sources.

The model used in the present study allows the assessment of behaviour during a period of acute histotoxic hypoxia. The treatment with $\mathrm{NaCN}$ disrupted the behaviour of young rats during a period of about $5 \mathrm{~min}$ post-injection; thereafter, the effects were no longer measurable. During this period drugs that are assumed to preserve neuronal function during a hypoxic state can be tested. Furthermore, after the behavioural experiments have been completed the effects of the $\mathrm{NaCN}$ treatment and a drug can be evaluated at a biochemical level in the same animals. This offers the possibility to correlate the behavioural and biochemical effects, which could lead to a better understanding of the mechanism of action of the drug tested. For example, we have evaluated the effects of ALCAR and NaCN injections on an energy-dependent process, namely lipid phosphorylation (in preparation).

In summary, the present study showed that chronic treatment with ALCAR improved the rate of learning in old rats. In young rats, an i.c.v. injection with $\mathrm{NaCN}(5.0 \mu \mathrm{g}$ bilateral) affected swimming behaviour in a Morris task, an effect probably due to a temporary loss of neuronal function during the period of metabolic inhibition. Chronic treatment with ALCAR reversed the $\mathrm{NaCN}$ induced behavioural deficit, which indicates that ALCAR preserves neuronal function during a short-lasting period of metabolic inhibition. ALCAR presumably increased the availability and the use of alternative energy sources during this period. Finally, the model used in the present study allows the assessment of behaviour during a period in which the energy level in the brain is lowered experimentally. This allows the assessment of the behavioural and biochemical effects of drugs that are assumed to preserve neuronal function during histotoxic hypoxia.

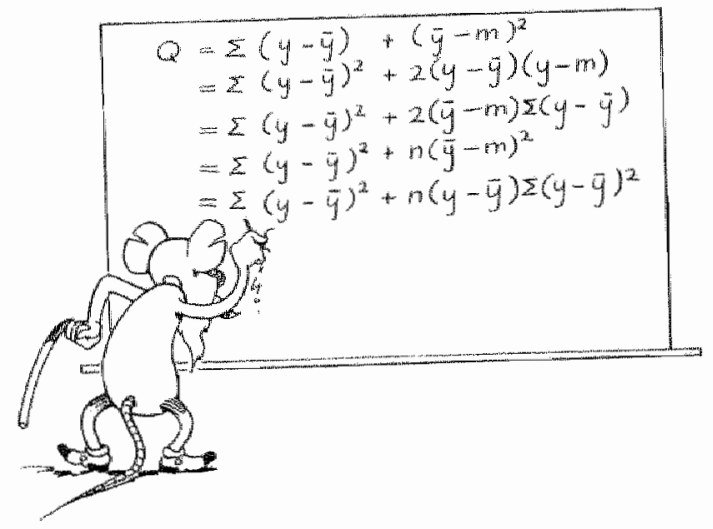




\section{SUMMARY AND CONCLUSIONS}

\section{Introduction}

The cognitive abilities of most people decline with increasing age. Many people experience increasing problems with retreiving information from memory and feel that this has a strong negatively influence on their quality of life. The decline in cognitive function is more dramatic in people suffering from dementia, which can be regarded as a case of pathological brain/cognitive aging. Because of the increased life span of man, the problems and diseases observed in aged people will become more prevalent. A better understanding of the processes that underlie the cognitive decline in aging and dementia could lead to better treatments and diagnostic tools. In addition, it may be possible to distinguish risk factors for cognitive functions in addition to the age-associated impairments of memory. Consequently, this type of research may eventually lead to an improvement in the quality of life of aged people. Although human studies have revealed some characteristics of the age-related cognitive decline, this research has practical and ethical limitations.

The cognitive decline in aging and dementia has been investigated in animals because there are fewer practical and ethical limitations. It is assumed that, when appropriate models are used, the results from animal studies can be extrapolated to man. In the last decade much attention has been focused on the development of valid animal models of cognitive aging. This research has revealed that the cognitive deficits observed in aged animals were to a great extent comparable to the cognitive deficits observed in aged people. However, the internal validity of animal models (but also human studies) can be questioned. An animal model of the age-related decline in cognitive function was evaluated in this dissertation. The performance of rats of different ages was assessed in different learning and memory tasks and by using different test procedures in these tasks. In addition, age-related differences in non-cognitive aspects of behavior were assessed in two different tests. Age-related changes in these non-cognitive behaviors could lead to differences in performance in learning tasks. By using one strain of rats and by measuring one dimension of learning and memory, these studies could lead to a better understanding of the nature of the age-related performance deficits in learning and memory tasks. This could give more information about the vallidity of currently used animal models of aging (i.e., spatial discrimination learning).

An animal model in which a fundamental biochemical process, the use of glucose in nerve cells, was affected was also evaluated in this thesis. A decrease in the availability of glucose in neurons has been considered as a possible cause for Alzheimer's disease. The behavioral effects of a decreased glucose availability were evaluated in an animal model of aging and performance was correlated with central cholinergic function. Finally, the behavioral effects of a nootropic drug were evaluated. Nootropic drugs are drugs which may alleviate the 
cognitive decline in aging and dementia. The effects of the drug on emotional reactivity were first evaluated. In a second experiment the effects of the nootropic drug were evaluated in a spatial discrimination paradigm. In this experiment the hypothesis that the nootropic drug could alleviate the availability of alternative energy sources was evaluated.

\section{Summary of studies}

\section{Chapter 2:}

In the first study a spatial cone-field apparatus was developed in which working memory and reference memory could be assessed simultaneously. The cone-field task also made it possible to measure aspects of behavior other than learning and memory (e.g, food search pattern) in order to control for factors that could interfere with learning and memory performance. The cone-field apparatus is considered a valuable tool in aging studies because different factors which may confound age-related performance deficits (speed of responding, motivation and food search patterns) can be controlled for. Different experiments were performed with the cone-field apparatus and a consistent age-related performance deficit in both working and reference memory was found. The conefield task could also be used in longitudinal studies. Taken together, the results suggested that the cone-field task could be regarded as a valuable task in aging research.

\section{Chapter 3:}

In the second study, Lewis rats of five different ages (4-, 12-, 18-, 24-, and 30 months) were tested in the cone-field task. The aim of the study was to evaluate the development of the age-related cognitive dlecline by using rats of five different ages covering the normal life-span. Unexpectedly, the results showed that from the age of 18 months onwards, Lewis rats had a better reference memory performance than younger rats whereas from the age of 12 months working memory performance was impaired. These results did not corroborate the general assumption of an age-related decline on both memory measures. The observation that 4-month-old rats had a superior working memory performance compared with rats of other ages suggested that they had adopted a habit of inspecting non-reinforced places. It was assumed that this was due to the procedure used in the adaptation phase of the cone-field task, in which all cones were baited. The younger (4-and 12-month-old) ratsinspected previously baited cones, which could have an ecological advantage. In addition, the results of the second study supported the notion that during aging different aspects of behavior decline at different rates.

\section{Chapter 4:}

In the third study young and old Lewis rats were tested in the cone-field apparatus but the task procedures were changed. The aim of this study was to evaluate whether old Lewis rats used an allocentric orientation strategy in the 
cone-field task. In this task the rats were started from one position and the cones were located in one corner in the cone-field. In accordance with other studies, no age-related differences in working and reference memory were found in the acquisition in this simple discrimination task. Moreover, the rats could have used an egocentric orientation strategy, an orientation strategy that does not decline with age. During probe trials, in which the rats were started from the opposite site of the cone-field, both the young and the old rats were found to use an allocentric orientation strategy. These findings indicate that the use of an allocentric orientation strategy does not necessarily decline with aging.

\section{Chapter 5:}

In the fourth study the performance of Lewis rats of three different ages was evaluated in another spatial discrimination paradigm. The rats were tested in the Morris spatial navigation task, a task that has revealed consistent age-related performance deficits in other studies. Because the reference memory performance of old Lewis rats was fairly high in the more complex cone-field task (see Chapters 3 and 4 ), it was assumed that old Lewis rats would perform the Morris task well. However, the old Lewis rats showed a marked escape performance deficit compared to the young and middle-aged rats in the Morris task. In a learning set procedure it was found that the performance of the old rats was not affected when the test environment was changed, whereas the escape latency of the younger rats increased during the first phase of training in a new environment. This suggested that the old Lewis rats did not use an allocentric orientation strategy in the Morris task. In an additional experiment it was found that old Lewis rats were only able to use a cue when it coincided with the platform position. The results of this study indicated that the learning and memory performance of young and old Lewis rats is task dependent. It was assumed that different operationalizations of learning (escape latency in Morris task; ratio of visits to baited and unbaited cones in coen-field), and differences in reinforcers (escape from water in Morris task; food in cone-field) contributed to the task dependent age-related performance differences. It was suggested that the aversive component of the Morris task (i.e., water) may have confounded the performance of the old Lewis rats.

\section{Chapter 6:}

Because the age-related differences in unlearned behavior (i.e., reactivity to a stressful stimulus) could have interfered with learning performance in the experiments described in Chapter 5 , the fifth study was designed to evaluate the age-related differences in reactivity to different stressful stimuli. Lewis rats and Brown-Norway rats of three different age groups were subjected to an open-field test. Analysis of two parameters of emotional reactivity revealed that the behavioral response to the novel environment was different for adult rats than for the middle-aged and old rats. A strain difference in emotional reactivity was also found, which corroborated the findings of other studies. In an additional experiment the same rats and young and old Wistar rats were subjected to an 
active avoidance task in a shuttle box. No consistent age-related performance deficit was found during acquisition training in the shuttle box. The expected strain difference in the acquisition of the shuttle box behavior was found. Because performance in the shuttle box is largely dependent on reactivity to the footshock, it was assumed that the age- and strain-dependent reactivity to shock could explain the findings of the shuttle box experiment. The results thus suggested that age-related differences in the unlearned behavioral response to stressful stimuli (e.g., novelty, shock, and water) could contribute to the agerelated difference in learning performance in learning tasks with an aversive component.

\section{Chapter 7:}

One other factor that could contribute to age-related performance deficits in learning tasks could be an age-related change in motivation to perform a task. A frequently used manner to motivate animals to acquire a task is to deprive them of food and to reward the required response with food. However, old rats weigh more than young rats and food deprivation may have a different effect in young and old rats. One method used to equalize the difference in food motivation between young and old rats is to reduce the percentual body weight of older rats more than that of younger rats. This technique has been named a differential deprivation technique. In the sixth study food motivation was assessed in a runway under two different deprivation levels. In addition, food motivation was assessed during a progressive ratio schedule of reinforcement at one level of deprivation. The results indicated that differential food deprivation can be considered a reliable method to achieve comparable levels of food motivation in rats of different ages. Furthermore, it was found that old rats needed more time to achieve maximal levels of performance in the runway, which suggested that old rats may need more training before their performance is optimal in learning tasks. Finally, no correlation was found between the operationalizations of motivation in the runway and in the progressive ratio test. This corroborated the notion that food motivation may not be a unitary concept and that other factors may interfere with notivation. Because differences in food motivation may have differential effects on learning performance, this factor should be controlled for, especially in tasks in which differences in motivation are likely to occur, e.g., aging studies.

\section{Chapter 8:}

The seventh study evaluated aging by using a within-subjects design. Young and old rats were subjected to different cognitive tasks and non-cognitive tests to evaluate the interdependence of the different behavioral parameters. In addition, the relation between behavior and three different biochemical measures was evaluated. Age-related differences in the relation between the different behavioral and/or biochemical measures could reveal information about the aging process. There were no correlations among the different parameters in and within the different tasks/tests, which underscores the notion that behavioral 
functions may decline independently and may be different for each subject. Food motivation and emotional reactivity did not predict performance in the learning tasks. Although there was no correlation between these non-cognitive behaviors and learning, it cannot be ruled out that these factors may contribute differently to the learning performance of young and old rats. Hippocampal choline acetyltransferase activity did not predict performance in the learning task in old rats, suggesting that this marker of cholinergic transmission may not be indicative of a cholinergic dysfunction in relation to aging. Baseline blood glucose levels correlated with the working and reference memory performance of old rats in the cone-field: no such correlation was found for young rats. The results suggested that the age-related decline in cognitive function may be related to an impaired regulation of blood glucose levels.

\section{Chapter 9:}

Most animal madels used to study the cognitive decline with age and dementia have evaluated the involvement of the cholinergic system by affecting this system directly, by lesioning cholinergic nuclei, or by affecting cholinergic transmission by injecting muscarinic antagonists. In the eighth study an animal model was introduced in which rats were treated with streptozotocin (injected intracerebroventricularly). Streptozotocin impairs the insulin-insulin receptor system in the brain and subsequently decreases the availability of glucose in nerve cells. It has been hypothesized that a decreased availability of glucose in nerve cells could be the underlying cause of Alzheimer's disease. Young rats treated with streptozotocin showed an impaired inhibitory avoidance performance but streptozotocin did not affect spatial discrimination learning in the Morris task as it does in rats treated with cholinergic antagonists. Thus the streptozotocin-treated rat cannot be regarded as an animal model of the cognitive decline associated with dementia because the spatial discrimination performance ogf the rats was not impaired at the end of training. However, middle-aged streptozotocin-treated rats showed an impaired spatial discrimination performance at the end of training in the Morris task comparable to that normally observed rats treated with cholinergic antagonists. Hippocampal choline-acetyltransferase activity was decreased in the middle-aged streptozotocin-treated rats. Hippocampal choline acetyltransferase correlated with the spatial discrimination performance of the middle-aged streptozotocintreated rats but not that of middle-aged control rats. These findings suggested that the middle-aged streptozotocin-treated rat could be regarded as an animal model of the cognitive decline of dementia. Furthermore, the data encourage further research into behavioral, biochemical and pathological aspects of the streptozotocin-treated rat.

\section{Chapter 10:}

In the ninth study the effects of a chronic treatment with the nootropic drug acetyl-1-carnitine on open-field behavior was evaluated in young and old rats. Acetyl-1-carnitine has been shown to alleviate learning impairments in old rats 
and to improve discrimination performance in adult rats. The aim of this study was to evaluate whether acetyl-J-carnitine also affected other aspects of behavior and whether the effects of the nootropic drug were age dependent. Old acetyl-1carnitine-treated rats were more emotionally reactive than the old control rats whereas treatment with acetyl-1-carnitine had no effect on the emotional reactivity of young rats. Although the results could suggest that the nootropic drug increased the emotional reactivity of old rats to a novel environment, this conclusion should be made with care. The operationalized measures of noveltyinduced emotional reactivity that were validated in young rats may not be valid measures of emotional reactivity in old rats (see Chapter 6).

\section{Chapter 11:}

In the last study an attempt was made to understand the possible mechanism of action of the nootropic drug acetyl-l-carnitine. An understanding of the mechanism of action of nootropic drugs may be of importance to further research for the development of cognition-enhancing drugs and possible treatments for aged and demented people. Young and old rats were chronically treated with acetyl-1-carnitine and trained in the Morris spatial navigation task. During training the escape latency of old acetyl-l-carnitine-treated rats decreased more rapidly than that of old control rats. However, the asymptotic performance of both groups of old rats was similar. This suggested that treatment with acetyl-1carnitine increased learning in old rats, which corroborates findings from other studies, but that it did not affect the use of a problem-solving strategy. After the rats had reached asymptotic performance, the effects of intracerebroventricular injections of sodium cyanide were evaluated. Cyanide induces a short-lasting period of histotoxic hypoxia. It was assumed that acetyl-l-carnitine would enhance the availability of alternative energy sources to preserve neuronal function during a period of hypoxia. This preservation of neuronal function was evaluated by assessing escape performance in the Morris task. No behavioral deficits were observed in young and old rats after a dose of $2.5 \mu \mathrm{g}$ of cyanide. This suggests that at this dose cyanide does not affect neuronal function in order to affect spatial orientation behavior. However, the escape performance in the Morris task was impaired 1 and 5 minutes after injection of $5 \mu \mathrm{g}$ cyanide in young rats. Chronic treatment with acetyl-l-carnitine attenuated the cyanideinduced behavioral deficit in young rats. The escape performance of old rats was not affected after $5 \mu \mathrm{g}$ cyanide. The absence of cyanide effects in old rats could be attributed to a systematic wrong cannula placement. Another possibility could be that old rats do not use an allocentric orientation strategy (see Chapter 5) and therefore cyanide may not affect this poor performance. The results suggest that an enhanced availability of energy sources may be the underlying mechanism for the alleviated learning performance in old rats. The paradigm could thus be useful in studying the behavioral and biochemical effects of neuroprotective agents. 


\section{Conclusions}

In the Chapters $2,3,4$ and 5 the performance of Lewis rats of different age groups was evaluated in two different spatial discrimination tasks using different procedures. No consistent age-related decline in cognitive function was found in Lewis rats of different ages (see also Chapter 6). This suggests that other factors could have contributed to the age-related difference in performance in different learning and memory tasks. It was assumed that young Lewis rats (up to an age of 12 months) adopted a habit of inspecting unbaited cones in the cone-field task and old Lewis rats (from an age of 18-20 months onwards) do not use an allocentric orientation strategy in the Morris spatial navigation task. These differences in the use of problem-solving strategies do not make it possible to interpret the differences in performance in learning tasks in terms of differences in spatial learning deficits. Apart from age-related differences in problem-solving strategies, there are other factors that could contribute to a difference in learning performance in rats of different ages.

Age-related differences in reactivity to stressful stimuli and food motivation were evaluated in this dissertation (Chapter 6 and 7 , respectively). In the study in which reactivity to stressful stimuli was evaluated in rats of different ages, the unlearned behavioral response to these stimuli was found to be expressed differently by rats of different ages. In the experiment in which age-related differences in food motivation were evaluated, we assumed that a differential food deprivation technique would lead to comparable levels of food motivation in rats of different ages. Taken together, the results of the Chapters 2 to 7 revealed that it is virtually impossible to compare age-related differences in learning and memory processes in learning and memory tasks. In the Introduction it was already mentioned that comparison of learning processes in rats of different age groups is impossible because of a definition of learning. Age-related differences in other aspects of behavior that could interfere with learning performance have been recognized by different authors (Ingram, 1985; Ray \& Barrett, 1973; Rigter, 1983; Sarter, 1987). Although these problems have been recognized, many studies still conclude that there are age-related differences in learning and memory in animals. Before such a conclusion can be drawn, one should be sure that the variables that could interfere differently with learning and memory performance in young and old rats are controlled for.

From the results of the Chapters 2 to 7 , in which predominantly one strain of rats (Lewis) was used, it can be concluded that the age-related performance deficit is task dependent and may not reflect differences in learning and memory capacity. The use of different tasks with different procedures can thus be considered as a powerful tool to investigate the nature of the age-related difference in performance in learning and memory tasks. Evaluation of noncognitive processes in rats of different ages may also contribute to a better understanding of the age-related differences in performance in learning and memory tasks. This leads to the first conclusion: 
Age differences in learning and memory performance should be evaluated in different tasks using different procedures. In addition, age-related differences should also be evaluated in noncognitioe tests. Conclusions with respect to learning and memory deficits should take the data obtained from the different tasks and tests into account.

Using this approach, one could say that conclusions cannot be drawn with regard to age-related learning and memory deficits in the rat, as has been shown for the Lewis strain in this dissertation.

This may lead one to conclude that it is not possible to compare age groups with respect to learning and memory processes. It may, however, be questioned whether it is necessary to evaluate differences in learning and memory between age groups. The primary aim of aging research is to understand the process of aging and to elucidate the possible causes of the age-related cognitive decline (see Introduction). From the Chapters 2 to 7 of this dissertation it was concluded that there are age differences in non-cognitive behaviors, and that these differences are likely to interfere with learning performance. It is therefore probable that a better understanding of age-related changes in more distinct (non)cognitive behavior may contribute to a better understanding of age-related changes in cognitive behavior.

It should also be mentioned that learning/memory is not a unitary concept but is consists of warious processes that contribute to the adaptive behavioral change. Until now, only little attention has been given to aspects of cognitive behavior that could underlie the age-related differences in learning and memory performance. It was shown recently that there is an age-related change in the use of problem-solving strategies (Caprioli et al., 1991). However, the performance of the rats was dependent on previous experience and was not dependent on the problem-solving strategy. Because a similar age-related change in the use of problem-solving strategies has been observed in humans (Zacks, 1982), experiments which evaluate age-related changes in problem-solving strategies in animals may reveal interesting data which may be of relevance to human studies.

Another cognitive process that shows a decline with age in man, i.e., attention (Giambra \& Quilter ${ }^{\text {i } 1988 ; ~ P l u d e ~ \& ~ D o u s s a r d-R o o s e v e l t, ~ 1989 ; ~ S t a n k o v, ~ 1988), ~ h a s ~}$ not been studied in animals thus far. The use of animals could be advantageous for evaluating the age-related decline in attentional processes. Evaluation of individual cognitive processes may lead to a better understanding of the agerelated decline in performance in learning and memory tasks. This leads to a second conclusion:
Research which evaluates the cognitive decline in aging and dementia should also consider the changes in (non)cognitive processes that could underlie the performance differences in learning and memory tasks. 
Another approach which may reveal more information about the aging process is the within-subject design (Chapter 8). The relation between different parameters of learning, non-cognitive behavior and biochemical measures gives information about parallel changes in behavior and biochemistry. It has been shown that the integrity of cholinergic neurons in the forebrain is required for spatial discrimination learning (Fischer et al., 1991), that the duration of bouts of paradoxical sleep is related to cognitive functions in old rats (Markowska et al., 1989), that brain glucose utilization is related to spatial discrimination learning (Gage et al., 1984b), and that blood glucose is related to spatial learning in old but not young rats (Chapter 8). Studies that correlate different aspects of behavior and biochemistry may reveal information about the relation between the deterioration of different functions with age. After having identified the relationship between behavior and/or blochemistry, further research could evaluate the causal relation between different parameters. A third conclusion is:

Correlational studies can reveal a relationship between behavior and biochemistry and consequently may contribute to our understanding of the aging process.

It was demonstrated in Chapter 9 that a decreased availability of brain glucose impaired the performance of middle-aged rats in the Morris spatial navigation task. Performance in the spatial discrimination task was related to hippocampal cholinergic function. These findings suggest that a fundamental biochemical process underlies the decrease in central cholinergic and cognitive function. This may explain why treatments with cholinergic drugs have not led to a consistent improvement in aged and demented people. A decrease in brain glucose metabolism (energy metabolism) has been found to be related to a decline in cognitive performance (see Gage et all., 1984b). Thus, it could be argued that alleviation of the decrease in brain energy metabolism could lead to an improvement in the cognitive functioning of aged and demented people. In the Chapters 10 and 11 the behavioral effects of a drug, acetyl-1-carnitine, were tested. This drug is assumed to increase the availability of alternative energy sources and therefore to preserve neuronal function. Different studies have shown that acetyl1-carnitine alleviates the learning and memory impairments of old rats (e.g., Caprioli et al., 1990; Ghirardi et all., in press). Because it was found that acetyl-1carnitine preserved neuronal function during a period of hypoxia, it was assumed that the mechanism of action of the drug is related to the energy metabolism of nerve cells. The observation that a decrease in brain energy levels could be a basal process underlying the cognitive deficits observed in aging and dementia and that drug which enhances brain energy metabolism, improves cognitive functioning supports further research of the fundamental biochemical processes affecting the energy level of the brain. The fourth conclusion:

The evaluation of fundamental processes which affect the energy state of the brain could be an important contribution to our 
understanding of the aging process and consequently lead to effective treatment for the cognitive decline associated with (pathological) aging.

\section{Summary of conclusions}

Age-related performance deficits in learning and memory tasks do not necessarily reflect age-related differences in learning and memory processes. A better insight into the nature of the age-related performance deficit can be obtained by using one strain of rats and by evaluating performance in animal models of cognitive aging that measures one dimension of memory. It is also important to evaluate other (non)cognitive processes which may interfere with learning and memory performance in order to understand the nature of the impaired performance in aged rats. Correlational studies in which behavioral and biochemical measures are evaluated may contribute significantly to our understanding of the aging process. Finally, behavioral and biochemical evaluation of the effects of basal biochemical processes may be a fruitful approach in aging research and could lead to treatments for the cognitive decline of aging and dementia. 


\section{REFERENCES}

- Adelstein, T.B. Kesner, R.P. \& Strassberg (1992). Spatial recognition and spatial order memory in patients with dementia of the Alzheimer's type. Neuropsycholagica, 30, 59-67.

- American Psychiatric Association. DSM-III-R: Diagnostic and statistical manual III (3rd ed., revised). APA Press: Washington D.C., 1987.

- Ando, S. \& Ohashi, Y. (1991). Longitudinal study on age-related changes of working and reference memory in the rat. Neurosci. Lett. 128, 17-20.

- Angelucci, L. \& Ramacci. M.T. Acetyl-l-carnitine: neuropharmacological potentialities in the

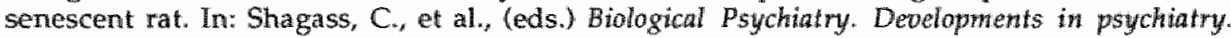
(vol. 7). Elseviers Science Publishing: New York,1986 (pp. 1349-1351).

- Aroujo, D.M., Lapchak, P.A. Meany, M.J., Collier, B. \& Quiron, R. (1990). Effects of aging on nicotinic and muscarinic autoreceptor function in the rat brain: relationship to presynaptic cholinergic markers and binding sites. J. Neurosci, 10, 3069-3078.

- Auer, R.N., Wieloch, T., Olsson, Y. \& Siesjö, B.K. (1984). The distribution of hypoglycemic brain damage. Acta Neuropathol., 64, 177-191.

- Aureli, T., Micchelli, A., Ricciolini, R., Enrica Di Cocco, M., Ramacci, M.T., Angelluci, L., Ghirardi, O. \& Conti, F. (1990). Aging brain: effect of acetyl-1-carnitine treatment on rat brain energy and phospholipid metabolism. A study by $31 \mathrm{P}$ and 1H NMR spectroscopy. Brain Res., 526 , 108-112.

- Azari, N.P. (1991). Effects of glucose on memory processes in young adults. Psychophatmacology, $105,521-524$.

- Badiali De Giorgi, L., Bonvicini, F., Bianchi, D., Bossoni, G. \& Laschi, R. (1987). Ultrastructural aspects of ageing rat hippocampus and effects of 1 -acetyl-camitine treatment. Drugs Exp. Clin, Res. $13,185-189$.

- Barnes, C.A. (1979). Memory deficits associated with senescence: A neurophysiological and behavioral study in the rat. J. Comp. Physiol. Psychol., 93, 74-104.

- Barnes, C.A. (1988). Spatial learning and memory processes: The search for their neurobiological mechanisms in the rat. Trends Neurosci., 11, 163-169.

- Barnes, C.A., Markowska, A.L., Ingram, D.K., Kametani, H., Spangler, E.L., Lemken, V.J. \& Otton, D.S. (1990). Acetyl-l-camitine 2: Effects on learning and memory performance of aged rats in simple and complex mazes. Neurobiol. Aging, 11, 499-506.

- Barnes, C.A., Nadel, L. Honig. W.K. (1980). Spatial memory deficit in senescent rats. Can. J. Psychol. / Reo. Car. Psychol., 34, 29-39.

- Bartus; R.T. Dean, R.L., Beer, B. \& Lippa, A.S. (1982). The cholinergic hypothesis of geriatric memory dysfunction. Science, $217,408-417$.

- Bartus, R.T. Dean, R.L., Pontecorvo, M.J. \& Flicker, C. (1985). The cholinergic hypothesis: a historical overview, current perspective, and future directions. In: Olion, D.S., Gainzw, E. \& Corkin, S. (Eds.), Memory dysfunctions: an integration of animal and human research from preclinical and clinical perspectives. Anm.N.Y.Acad. Sci, 444, 332-358.

- Baughman, V.L., Hoffman, W.E., Miletich, D.J. \& Albrecht, R.F. (1990). Cerbrovascular and cerebral metabolic effects of $\mathrm{N}_{2} \mathrm{O}$ in untestrained rats. A nesthesiology. $73,269 \mathrm{~m} 272$.

- Bierley, R.A., Ruxem, G.J., Tröster, A.I., \& Bealty, W.W. (1986). Preserved spatial memory in old rats survives 10 months without training. Behat. Neur. Biol., 45, 223-229.

- Behroozi, K.R., Guttman, R., Gruener, N. \& Shu val, H.1. (1972). Changes in motor activity of mice giwen sodium nitrite in drinking solution. Istadien J. Med. Sci., 8, 1007.

- Birren, J.E. \& Schaie, K.W. Handbook of the psychology of aging. (2nd ed.). Van Nostrand Reinhold Company: New York, 1985.

- Blokland, A, Raaijmakers, W., van der Staay, F.J. \& Jolles, J. (1990). Differential effect of Acetyl1-carritine on open field behavior in young and old rats. Phystol. Behaw., 47, 783-785.

- Blokland A., Honig, W.. \& Raaijmakers, W. Age-related changes in spatial discrimination 
learning in Lewis rats. (submilted).

- Bolles, R.C. Theory of motivation. (2nd ed.) Harper and Row: New York, 1975a.

- Bolles, R.C. Leaming theory. Holt, Rinehart and Winston: New York: 1975b.

- Botwinnick, I. Neuropsychology of aging. In: Filskov, S.B. \& Boll, T.J. (Eds.). Handbook of

Clinical Neuropsychology. Wiley: New York, 1981 (135-171).

- Bowen, D.M. Smith, C.B., White, P. Davison, A.N. (1976). Neurotransmitter-related enzymes and indices of hypoxia in senile dementia and other abiotrophes. Brain, $99,459-496$.

- Bower, G.H. \& Hilgard, E.R. Theories of learning (5th ed). Prentice-Hall Inc. Englewood Cliffs N..., 1981.

- Brierly, J.B., Brown, A.W. \& Calverley (1976). Cyanide intoxication in the rat: physiological and neuropathollogical aspects. I. Neur. Neurosurg. Psychiat., 39, 129-140.

- Broadhurst, P.L. (1957). Determinants of emotionality in the rat. I. Situational factors. Br. $)$

Psychol. 48, 1-12.

- Broks, P., Preston, G.C., Iraub, M., Poppleton, P., Ward, C. \& Stahl, S.M. (1988). Modelling

dementia: Elfects of scopolamine on memory and attention. Neuropsychologica, 26, 685-700.

- Burelk, J.D. Pathology of Aging Rats. CRC Press, Inc.:West Palm Beach, 1978.

- Cahill, L. \& McGaugh, J.L. (1990). Amygdaloid complex lesions differentially affect retention of tasks using appetitive and aversive reinforcement. Behat. Neurosci., 104,532-543.

- Campbell, B.A. \& Haroutunian, V. (1981). Effects of age on long-term memory: retention of fixed interval responding. I. Gerontol., 36, 338-341.

- Campbell, B.A., Teghtsoonian, R. \& Williams, R.A. (1961). Activity, weight loss, and survival time of food-deprived rats as a function of age. I. Comp. Physiol. Psychol.,54, 216-219.

- Caprioli, A., Ghirardi, O., Giuliani, A., Ramacci, M.T. \& Angelucci, L. (1991). Spatial learning and memory in the radial maze: a longitudinal study in rats from 4 to 25 months of age. Neurobiol. Aging, 12, 605-607.

- Caprioli, A., Ghirardi, O., Ramacci, M.T. \& Angelucci, L. (1990). Age-dependent deficits in radial maze performance in the rat: effect of chromic treatment with acetyl-1-carnitine. Prog.

Neuropsychopham. Biol. Psychiat., 14, 359-369.

- Coleman, P.D. (1989). How old is old? Newrobiol. Aging, 10, 115.

- Coleman, P., Finch, C. Joseph, ]. (1990). The need for multiple time points in aging studies.

Neurobiol. Aging, 11, 1-2.

- Collerton, D. (1986). Cholinergic function and intellectual decline in Alzheimer's disease.

Neuroscience, 19, 1-28.

- Cooper, A.J.L. \& Plum, F. (1988). Biochemistry and physiology of brain ammonia. Physiol. Rev., 67, 440-519.

- Craik, F.J.M. Age differences in human menory. In: Birren, J.E. \& Shaie, K.W. (Eds.) Handbook of the psychology of aging. Van Nostrand Reinhold Company: New York, 1977 (pp. 384-420).

- Craft, S., Zallen, G. \& Baker, L. D. (1992). Glucose and memory in mild senile dlementia of the Alzheimer type. Journal of Clinical and Experimental Neuropsychology, 14, 253-267.

- Crane, P.D., Braun, L.D. Cornford, E.M. Cremer, J.E., Glass, J.M. \& Oldendorf, W.H. (1978). Dosedependent reduction of glucose utilization by pentobarbital in rat brain. Stroke, 9, 12-18.

- Crook, T., Bartus, R.T., Ferris, S.H., Whitehouse, P., Cohen, G.D. \&ershon, S. (1986). Ageassociated memory impairment: Proposed diagnostic criteria and measures of clinical change report of a national institute of mentall health work group. Develop. Newropsychol, $2,261-276$. - Curti, D. Dagani, F., Marzatico, F. \& Benzi, G. Aging and acelyl-l-camitine treatment: effects on some cerebral biochemical parameters. In: Shagass, $C_{\text {, }}$ et al. (Eds.) Biological Psychiatry.

Developments in psychiatry. (vol. 7) Elseviers Science Publishing: New York, 1986 (pp. 1343-1345).

- Dean, R.L. \& Bartus, R.T. Behavioral models of aging in nonhuman primates. In: Iversen, L.L."

Iversen, S.D. S Snyder, S.H. (Eds.), Psychopharmacology of the aging nervous system (Handbook of psychopharmacology, vol. 20). Plenum Press: New York, 1988 (pp. 325-392).

- de Boer, S.F., Koopmans, S.I. Slangen, J.L. \& Van der Gugten, J. (1990). Plasma catecholamine, corticosterone and glucose responses to repeated stress in rats: effect of interstressor interwal length. 
Physiol. Behad, 47, 1117-1124.

- Decker, M.W., Pelleymounter, M.A. \& Gallagher, M. (1988). Effects of training on a spatial memory task on high affinity choline uptake in hippocampus and cortex in young adult and aged rats. I. Neurosci: $8,90-99$.

- deLeon, M.J., Harris, S.H., George, A.E., Reisberg, B., Christman, D.R. Kricherf, I.I. \& Wolk A.P. (1983). Computed tomography and positron emission transaxial tomography evaluation of normal aging and Alzheimer"s Disease. J. Cerebr. Blowd Flow Met, 3, 391-394.

- de Ryck, M. (1990). Animal models of cerebral stroke. pharmacological protection of function. Europ. Neurol., 30, 21-27.

- Devenport, L.D. \& Cater, N. (1986). Etharol blockade of context change effects. Behav. Neural Biol., 45,135-142.

- Ding, A.. Nitsch, R. \& Hoyer, S. (1992). Changes in brain monoaminergic neurotransmitter concentrations in rat after intracerebroventricular injection of streptozotocin. J. Cereb. Blood Flow Mer. 12, 103-109.

- D'Mello, G.D. (1986). Effects of sodium cyanide upon swimming performance in guinea-pigs and the conferment of protection by pretreatment with $\mathrm{p}$-aminopropiophenone. Neturbbehav. Toxicol. Teratol., 8, 171-178.

- Doty, B.A. (1966). Age differences in avoidance conditioning as a function of distribution of trials and task difficulty. I. Genet. Psychol. 109, 249-254.

- Doty, B.A. (1972). The effects of cage environment upon avoidance responding of aged rats. 1 . Gerontol, 27, 358-360.

- Drachman, D.A. \& Leavitt, J. (1974). Human memory and the cholinergic system. Arch. Newrol., 30, 113-121.

- Drago, F, Continella, G., Pennisi, G., Alloro, M.C., Callani, M. \& Scapagnini, U. (1986\%.

Behavioral effects of acetyl-1-carnitine in the male rat. Pharmacol. Biochem. Behav. "24, 1393-1396.

- Dunntett, S.B. (1985). Comparative effects of cholinergic drugs and lesions of the nucleus basalis or fimbria-fornix on delayed matching in rats. Psychophamacology, 87, 357-363.

- Dunnett, S.B., Evenden, J.L. \& Iversen, S.D. (1988). Delay-dependent short-term menory deficits in aged rats. Psychopharmacology, 96, 174-180.

- Dunnett, S.B., Everitt ${ }_{s}$ B.J. \& Robbins, T.W. (1991). The basal forebrain-cortical system:

interpreting the functional consequences of exitotoxic lesions. Trends Neurosci., 14, 494-501.

- Elias, M.F. \& Elias, P.K. Motivation and activity. In: Age, leaming ability and intelligence.

Sprott, R.L. (Ed.). Van Nostrand Reinhold: New York, 1977 (pp. 26-40).

- Fibiger, H.C. (1991). Cholinergic mechanisms in learning memory and dementia: a review of recent evidence. Trends Netrosci., 14, 220-223.

- Fischer, W., Chen, K.S., Gage, F.H. \& Björklund, A. (1991). Progressive diecline in spatial learning and integrity of forebrain cholinergic neurons in rats duning aging. Newrobiol. Aging, 13, 9-23.

- Flicker, C., Ferris, S.H., Crook, T., Bartus, R.T. \& Reisberg, B. Cognitive function ín normal aging and early dementia. In: "Traber, ${ }_{*}$. \& Gispen, W.H. (Eds.), Senile dementia of the Alzheimer type. Springer-Verlag (Berlin): Heidelberg, 1985 (pp. 2-17).

- Fonnum, F. (1975). A rapid radio-chemical method for the determination of choline acetyltransferase. ]. Neurochem, $24,407-409$.

- Fritz, I.B. (1963). Carnitine and its role in fatty acid metabolism. Adv. Lipid Res., 1, 285-334.

- Fry, W., Kelleher, R.T. \& Cook (1960). A mathematical index of performance on fixed-interval schedules of reinforcement. 1. Exp. Anual. Behav., 3, 193-199.

- Gage, F.H., Chen, K.S., Buzsaki, G. Armstrong, D. (1988). Experimental approaches to age-related cognitive impairments. Neurobiol. Aging, 9, 645-655.

- Gage, F.H., Dunnett, S.B. \& Björklund, A. (1984a). Spatial learning and motor deficits in aged rats. Newrobiol. Aging, 5, $43-48$.

- Gage, F.H., Kelley, P.T.A. B Bjöklund, A. (1984b). Regional changes in brain glucosta metabolism reflect oognitive impairment in aged rats. J. Newrosci., 4, 2856-2865. 
- Gage, F.H., Dunnett, S.B. \& Björklund, A. (1989). Age-related impairments ín spatial memory are independent of those in sensorimotor skills. Newrobiol. Aging, 10, 347-352.

- Gallagher, M. \&elleymounter, M.A. (1988a). An age-related spatial learning deficit: choline uptake distinguishes "impaired" and "unimpaired" rats, Newrobiol. Aging, 9, 363-369.

- Gallagher, M. \& Pelleymounter, M.A. (1988b). Spatiall learming deficits in old rats: A model for decline in the aged. Neurobiol. Aging, 9, 549-556.

- Gamzu, E. (1985). Animal behavioral models in the discovery of compounds to treat memory dysfunction. In: Olton, D.S., Gamzu, E. C Corkin, S. (Eds.), Memory dysfunctions: an integration of animal and human research from predinical and clinical perspectives. Ann. N. Y. Acad. Sci., 444, $370-393$.

- Ghirardi, O, Milano, S., Ramacci, M.T. \& Angelucci, L. (1988). Effect of acetyl-1-camitine chronic treatment on discrimination models in aged rats. Physiol. Behad:, 44, 769-773.

- Ghirardi, O. Milano, S., Ramacci, M.T. \& Angelucci, L. (in press). Acetyl-1-carnitine and spatial learning in senscent rats. Prog. Neuropsychophamacol. Biol. Psychiat.

- Gtambra, L.M. Q Quilter, R. (1988). Sustained attention in adulthood: a unique, large sample, longitudinal and multicohort analysis using the Mackworth-Clock-Test. Psychol. Aging, 3, 75-83.

- Gibson, G.E., Pelmas, C.J. \& Peterson, C. (1983). Cholinergic drugs and 4-aminopyridine alter hypoxic-induced behavioral deficits. Pharmacol. Biochem. Behav, 18, 909-916.

- Gibson, G.E. \& Peterson (1981). Aging decreases metabolism and the release and synthesis of acetylcholine. I. Neurochem., 37, 978-984.

- Gilad, G.M. \& Shiller, 1. (1989). Differences in open-field behavior and in learning tasks between two rat strains differing in their reactivity to stressors. Behav. Brain Res., 32, 89-93.

- Giurgia, C.E., Greind1, M.G. \& Preat, S. (1983). Nootropic drugs and aging. Acta Psychiat. Bel., 83, 349-358.

- Goma, M. \& Tobena A. (1978). Reliability of various measures obtained in open-field test.

Psychol. Reports, 43,559-569.

- Goodrick, C.L. (1968). Learning, retention, and extinction of a complex maze habit for

mature-young and senescent Wistar albino rats. J. Gerontol., 27, 298-304.

- Goodrick, C.L. (1972), Learning by mature-young and aged Wistar rats as a function of test

complexity. I. Gerontol, $27,353-357$.

- Goodrick, C.L. (1973). Maze learning of mature-young and aged rats as a fumction of distribution of practice. \%. Exp. Psychol, 9.8, 344-349.

- Goodrick, C.L. (1975). Behavioral rigidity as a mechanism for facilitation of problem solving for aged rats. 1. Gerontol., 30, 181-184.

- Goodrick, C.L. Problem solving and age: A critique of rodent research. In: Sprott, R.L. (Ed.), Age*

learming ability, and intelligence. Van Nostrand Reinhold: New York, 1980 (pp. 5u25).

- Hagan, J. I. Morris, R.G.M. The cholinergic hypothesis of memory: A review of animal

experiments. In: Iversen, L.L., Iversem, S.D. \& Snyder, S.H. (Eds.) Handbook of

psychopharmacology (vol. 20). Plenum Press: New York, 1988 (pp. 237-323).

- Hall, C.S. (1936). Emotional behavior in the rat. III. The relationship between emotionality and ambulatory activity. 1. Comp. Physiol. Psychol, 22, 345-352.

- Hall, J.L., Gonder-Frederick, L.A., Chewning, W.W., Silveira, J. \& Gold, P.E. (1989). Glucose enhancement of memory in young and aged humans. Newropsychologica, 27, 1129-1138.

- Hamm, R.J. Knisley, J.5. \& Dixon, C.E. (1983). Animal models of age changes in short-term memory: the DRL schedule. Exp. Aging Res, 9, 23-25.

- Hathway, D.E.. Molecular aspects of toxicology. The Royal Society of Chemistry: London, 1984, p. 84.

- Hepler, D.J., Wenk, G.J. Cribbs, B.L., Olton, D.S. \& Coyle, J.T. (1985), Memory impairments following basal forebrain lesions. Brain Res, $346,8-14$.

- Hock, F.J. (1987). Drug influences on learning and memory in aged animals and humans.

Nenropsychobiology, 17, 145-160.

- Hodos, W. (1961). Progressive ratio as a measure of reward strength. Science, 134, 943-944. 
- Houx, P.) (1991). Cognitioe aging and health-related factors. Doctoral dissertation, University of Limburg, The Netherlands.

- Hoyer, S. \& Krier, C. (1986). Ischemia and the aging brain. Studies on glucose and energy metabolism in rat cerebral cortex. Neurobil. Agizg. 7, 23-29.

- Ingram, D.K. (1985). Analysis of age-related impairments in learning and memory in rodent models. In: Oiton, D.S., Gamzu, E. \& Corkin, S. (Edss.). Memory dysfunctions: an integration of animal and human research from preclinical and clinical perspectives. Anm. N. Y. Acad. Soi, 444 , $312-331$.

- Ingram, D.K. (1988). Complex maze learning in rodents as a model of age-related memory impairment. Neurobiol. Aging, 9, 475-485

- Ingram, D.K., London, E.D. \& Goodrick, C.L. (1981). Age and neurochemical correlates of radial maze performance in rats. Neurobiol. Aging, 2, 1147 .

- Ingram, D.K., Spangler, E.L. \& Vincent, G.P. (1983). Behavioral comparison of aged virgin and retired breeder mice. Exp. Aging Res, 9, 111-113.

- Jarrard, L.E. (1980). Selective hippocampal lesions and behavior. Physiol. Psychol, 8, 198-206. - Jarrard, L.E., Okaichi, H. Steward, O. \& Goldschmidt, R.B. (1984). On the role of hippocampal connections in the performance of place and cue tasks: Comparisons with danage to hippocampus. Behary. Neurosci., 98, 946-954.

- Johnson, M.K. \& Hasher, L. (1987). Human learning and memory. Anr. Rev. Psychol, 38, 631-668. - Jolles, J. (1983). The pharmacology of memory. Intermal Med, 3, 2-5.

- Jolles, J. Neuropeptides and the treatment of cognitive deficits in aging and dementia. In: Swaab. D.F., Fliers, E., Mirmiran, M., Van Gool, W.A. \& Van Haaren, F. (Eds.). Prog. Brain Res, , 70, Elsevier: Amsterdam, 1986 (429-441).

- Jolles, J., Bothmer, J., Markerink, M. \& Ravid, R. (1992). Phosphatidylinositol kinase activity is reduced in Alzheimer"s disease. I. Neurochem., 58, 2326-2329.

- Jucker, M., Oettinger, R. \& Bältig, K (1988). Age-related changes in working and reference menory performance and locomotor activity in the Wistar rat. Behav. Neur. Biol., 50, 24-36.

- Katzman, R. (1986). Alzheimer's disease. N.Eng. J. Med., 314, 964-973.

- Kobayashi, S., Kametani, H., Ugawa, Y., \& Osani, M. (1988). Age difference of response strategy in radial naze performance of Fischer-344 rats. Physiol. Bekav., 42, 277-280.

- Kolb, B. \& Whishaw, I.Q. Furdametatals of thuman mewropsychology (3rd ed.). Freeman and Co.: New York, 1990 (pp. 643-676).

- Kominsky, H.L., Buck, M.A. Mundinger, K.L., McSweeney, F.K., Farmer-Dougan, V.A. \& Dougan, J.D. (1987). Effects of aging on anticonflict and CNS depressant activity of diazepam in rats.

Psychopharmacology, 93, 443-448.

- Lachman, S.]. \& Brown, C.R. (1957). Behawior in a free choice multiple path elimination problem, 1. Psychols, 43, 27-40.

- Levenson, R.W., Carstensen, L.L., Friesen, W.V. \& Ekman, P. (1991), Emotion, Physiology, and expression in old age. Psychol. Aging, 6, 28-35.

- Levere, "T.E. \& Walker, A. (1991). Old age and cognition: enhancement of recent memory in aged rats by the calcium entry blocker nimodipine. Neurabiol. Aging, 13, 63-66.

- Light, L.L. (1991). Memory and aging: four hypothesis in search of data. Ann. Rev. Psychol, 42, 333-376.

- Lindner, M.D. \& Schallert, T. (1988). Aging and atropine effects on spatial navigation in the Morris water task. Behav. Neurosci, 102, 621-634.

- Lindsay, S., Nichols, C.W. \& Chaikoff, LL. (1968). Naturally occurring thyroid carcinoma in the rat. Similarities to human medullary carcinoma. Arch. Pathol, $86,353-364$.

- Lowy, A.M., Ingram, D.K., Ollon, D.S., Waller, S.B., Reynolds, M.A. London, E.D. (1985).

Discrimination learning requiring different memory components in rats: Age and neurochemical comparisons. Behav. Neurosci., $99,638-651$.

- Luine, V. Hearns, M. (1990). Spatial memory deficits in aged rats: contributions of the cholinergic system assessed by ChAT. Brain Res, 523, 321-324. 
- MacMillan, V.H. (1989). Cerbral energy metabolism in cyanide encephalopathy. I. Cereb. Blood Flow Metabol, 9, 156-162.

- Markowska, A.L., Ingram, D.K., Barnes, C.A., Spangler, E. L., Lemken, V.J., Kametani, H., "Yee, W. Olton, D.S. (1990). Acetyl-I-cannitine 1: Effects on mortality, pathology and sensory-motor performance in aging rats. Neurobiol. Aging, 11, 491498.

- Markowska, A.L. Stone, W.S., Ingram, D.K., Reynolds, I., Gold, P.E., Conti, L.H., Pontecorvo, M.I., Wenk, G.L. \& Olton, D.S. (1989). Individual differences in aging; behavioral and neurobiological correlates. Neurobiol. Aging, 10, 31-43.

- Marshall, J.F. (1982). Sensorimotor disturbances in the aging rodent. J. Gerontol, 37, 548-554.

- Mayer, G., Nitsch, R. \& Hoyer, S. (1990). Effects of changes in peripheral and cerebral glucose metabolism on locomotor activity, learning and memory in adult male rats. Brain Res, 532, 95-100. - MoCarty, R. \& Kopin, 1.). (1978). Sympatho-adrenal medullary activity and behavior during exposure to fooshock stress: a comparison of seven rat strains. Physiol. Behav., 21, 567-572.

- MoGeer, P.L. \& MoGeer, E.G. Aging and neurotransmitter systems. In: Finch, C.E., Potter, D.E. \& Kenny (Eds.). Parkinson's disedse II. Adonaces in experimental medicine and biology, (113). Plenum Press: New York, 1978 (pp. 411-47).

- Morris, R.G.M. (1981). Spatial localization does not require the presence of local cues. Learn. Matio., 12, 239-261.

- Morris, R.G.M., Garrud, P., Rawlins, I.N.P. \& O'Keefe (1982). Place navigation impaired in rats with hippocampal lesions. Nature, 297, 681-683.

- Morris, R.G.M., Schenk, F., Tweedie, F. \& Jarrard, L.E. (1990). Ibotenate llesions of hippocampus and/or subiculum: dissociating components of allocentric spatial leaming. Eur. J. Neurosci., 2, 10161028.

- Munn, N.L. Handbook of psychological research on the ret. Houghton Mifflin Company: Boston, 1950.

- Nitsch, R. \& Hoyer, S. (1991). Local action of the diabetogenic drug, streptozotocin, on glucose and energy melabolism in rat brain cortex. Neurosci. Lett., 128, 199-202.

- Nitsch, R., Mayer, G. \& Hoyer, S. (1989). The intracerebroventricularly streptozotocin-treated rat: impairment of cerebral glucose metabolism resembles the alterations of carbohydrate metabolism of the brain in Alzheimer's disease. J. Neur. Transm. [P-D Section], 1, 109-110.

- Oades, R.D. Isaacson, R.L. (1978). The development of food search behavior by rats: The effects of hippocampal damage and haloperidol. Behav. Biol, 24, 327-337.

- Ohtsuka, Y. \& Griffith, O.W. (1991). 1-Carnitine protection in ammonia intoxication. Biochem.

Pharmacol., 41, 1957-1961.

- O'Keefe, I. \& Nadel, L. The hippocampus as a cognitive map. Clarendon Press: Oxford, 1978.

- Olton, D.S. (1977). Spatial memory. Scientific American, 236, 82-98.

- Olton, D.S. Memory functions and the hippocampus. In: W. Seifert (Ed.), Neturobiology of the Hippocampusis Academic Press: London, 1983 (pp. 335-373).

- Olton, D.S. (1988). Aging and spatial cognition. Newrobiol. Aging, 9, 569-570.

- Olton, D.S. Becket, J.T. \& Handelmamn, G.F. (1979). Hippocampus, space, and memory. Behav.

Brain Sci, 2, 313-365.

- Olton, D.S., Collisom, C. \& Werz, M.A. (1977). Spatial memory and radial maze performance of rats. Learm. Motiv., 8, 289-314.

- Olton, D.S., Markowsika, A.L. (1988). Within-subjects, parametric mamipulations to investigate aging. Neurobiol. Aging, 9, 469-474.

- Olton, D.S. \& Papas, B.C. (1979). Spatial memory and hippocampal function. Neuropsychologica, $17,669-682$.

- Olton, D.S. Samuelson, R.J. (1976). Remembrance of places passed: Spatial memory in rats. J.

Exp. Psychol: Anim. Behtavi. Process, 2, 97-116.

“ Onofrj, M., Bodis-Wollner, I., Pola, P. \& Calvani, M. (1983). Central cholinergic effects of levo-acetylcarnitine. Drugs Exp. Clin. Res. , 9, 161-169.

- Ossenkop, K.P. and Mazmanian, D.S. (1985). The principle of aggregation in psychobiological 
correlational research: An example from the open-field test. Awim. Leam. Beltav, 13, 339-344. - Paxinos, G. \& Walson, C. The rat brain in stereotaxic coordinates. (2nd edition) Academic Press: Sydney, 1986.

- Pelleymounter, M.A. Smith, M.Y. \& Gallagher, M. (1987). Spatial leaming impairnents in aged rats trained with a salient configuration of stimuli. Psychobiology, $13,284-354$.

- Peruche, B., Ahlmeyer, B., Brungs, H. \& Krieglstein, J. (1990). Cultered neurons for testing antihypoxic drug effects. I. Phamracol. Meth, $23,63-77$.

- Perry, E.K. (1980). The cholinergic system in old age and Alzheimer's disease. Age Ageing, 9, 1-8.

- Plude, D.J. \& Doussard-Roosevelt, J.A. (1989). Aging, selective attention, and feature integration. Psychol. Aging, 4, 98-105.

- Pörtner, H.O., Boutilier, R.G., Tang, Y. \& Toews (1990). Determination of intracelular pH and

$P_{\mathrm{CO}}$ after metabolic inhibition by fuoride and nitrilotriacetic acid. Respir. Physiol, 81, 255-274.

- Rapp, P.R., Rosenberg, R.A. \& Gallagher, M. (1987). An evaluation of spatial information

processing in aged rats. Behav. Newrosci., 101, 3-12.

- Ray, O.S. Barrett, R.J. Interaction of learning and memory with age in the rat. In:

Psychophamacology and aging. Eisdorfer, C. Bann, W.E. (Eds.). Plenum Press: New York, 1973 (pp. 17-39).

- Rigter, H. Pitfalls in behavioural ageing research in animals. In: Aging of the Brain. Gispen, W.H. \& Traber, I. (Eds.). Elsevier: Amsterdiam, 1983 (pp. 197 208).

- Salamone, J.D. (1986). Behavioral functions of the nucleus basalis magnocellularis and its relationship with dementia. Trends Neurosci. June, $256-258$.

- Salthouse, T.A. Speed of behavior and its implications for cognition. In: Birren, JE. Shaile, K.W. (Eds.), Handbook of the psychology of aging. (2nd ed.). Van Nostrand Reinhold Company: New York, 1985 (pp. 400-426).

- Salthouse, T.A. (1988). Initiating the formalization of theories of cognitive aging. Psychol. Aging. 3, 3-16.

- Salthouse, T.A. Cognition, motor behavior, and the assessment of atypical aging. In: Howe, M.L., Stones, M.J. \& Brainerd, C.J. (Eds.). Cognitive and behavioral perfornance factors in atypical aging. Van Nostrand Reinhold: New York, 1990 (pp. 400-426).

- Sapolsky, R., Krey, L. \& McEwen, B. (1983). The adrenocortical stress-response in the aged male rat: impairment of recovery from stress. Exp. Gerontol., 18, 55 64 .

- Sarter, M. (1987). Measu rement of cogmitive abilities in senescent animals. Int. I. Newrosci., 32, 765774.

- Sarter M. (1991). Taking stock of cognitive enhancers. Trends Pharmacal Sci, 12, 456-461.

- Sarter, M. \& Markowitsch, H.J. (1983). Reduced resistance to progressive extinction in senescent rats: A neuroanatomical and behavioral study. Newrobiol. Aging, 4, 203-215.

- Schoeder, F. (1984). Role of membrame lipid asymmetry in aging. Newrobiol. Aging, 5, 323-333.

- Schuurman, T. \& Traber, I. Old rats as an animal for senile dementla: Behavioral effects of nimodipine. In: Bergener, M. \& Reisberg, B. (Eds.) Diagnosis and treatment of sichile dewiewtia. Springer-Verlag: Berlin, 1989.

- Sprangler, E.L., Chachich, M.E., Curtis, N.J. \& Ingram, D.K. (1989). Age-related impairment in complex maze learning in rats: relationship to neophobia and cholinergic antagonism. Neurobiol. Aging, 10, 133-141.

- Stankov, L. (1988). Aging, attention, and intelligence. Psychol. Aging, 3, 59-74.

- Stone, C.P. (19.29). Age factor in animal learning. Gen. Psychol. Mort., 5, 1-130.

- Stone, W.S., Cottrill, K.L., Walker, D.L. \& Cold, P.E. (1988). Blood glucose and brain function: Interaction with CNS cholinergic systems. Behav. Neur. Biol., 50, 325-334.

- Stone, W.S. Wenk, G.L., Olton, D.S. \& Gold, P.E. (1990). Poor blood glucose regulation predicts sleep and memory deficits in normal aged rats. I. Gerontol., 45, B169-173.

- Terwel, D. Markerink, M. \& Jolles, J. (1992). Peptidases are affected differendly in neocortical regions of brains from patients with Alzheimer's disease. Dementia, 3, 108-113.

- Van der Staay, F.I. Behavioral consequences of chromic dietary cholne enrichment. Doctoral 
dissertation, University of Nijmegen, 1989.

- van der Staay, F.J., Blokland, A. \& Raaijmakers, W.G.M. (1990a). Different time course for agerelated changes of behavior in a complex spatial cone-field discrimination task in Lewis rats.

Psychobiology, 18, 305-311.

- Van der Staay, F.J. Kerbusch, S. Raaijmakers, W.G.M. (1990b). Genetic correlations in wallidating emotionality. Behav. Genet, 20,51-62.

- van der Staay, F.J., Krechting, B., Blokland, A. \& Raatimakers, W.G.M. (1990c). The cone field: A spattal discrimination taskl for the automatic and simultaneous assessment of working and reference memory in rats. \%. Neurosci. Meth, 31, 13-22.

- van der Staay, F., Raaijmakers, W.G.M. \& Collijn, T.H. (1986). Spatial discrimination and parsive avoidance behavior in rats: Age-related changes and modulation by chronic dietary choline enrichment. In: A. Fisher, I. Hanin; \& C. Lachman (Eds.). Alzheimer's and Parkinson's diseases: Strategies for research and development. Adv. Behav. Biol, 29: 603-608.

- wan der Staay, F.J., Raaijmakers, W.C.M., Lammers, A.J.J.C. \& Tonnaer, J.A.D.M. (1989). Selective fimbria lesions impair acquisition of working and reference memory of rats in a complex

discrimination task. Behav. Brain Res., 32, 151-161.

- van der Staay, F.J., Raaijmakers, W.G.M., Sakkee, A.N. \& wan Bezooijen, C.F.A. (1988). Spatial working and reference memory of adult and senescent rats after thiopental anaesthesia. Neurosci.

Res. Comm. 3, 55-61.

- van der Staay, F.J., wan Nies, J. \& Raaijmakers, W. (1990d). The effects of aging in rats on working and reference memory performance in a spatial holeboard discrimination task. Behav. Newr. Biol. $53,356-370$.

- van Hest, A, Van Haaren, F. \& Van de Poll, N.E. (1988). The behavior of male and female rats pressing a lever for food is not affected by sex differences in food motivation. Behav. Brain Res., 27, 215-221.

- van Luijtelaar, E.L.J.M, van der Staay, F.J. \&erbusch, J.M.L. (1989). Spatial memory in rats: A cross validation study. Quart. J. Exp. Psychol., 41B, 287-306.

- Wallace, J.E., Krauter, E.E. Campbell, B.A. (1980). Animal models of declining memory in the aged: short-term and spatial memory in the aged rat. J. Gerontol, 35, 364-370.

- Way, J.L. (1984). Cyanide intoxication and its mechanisms of antagonism. Ann. Reo. Phamacol.

Toxicol., 24, 451-481.

- Weijnen, J.A.W.M. \& Slangen, J.L. (1970). Effects of ACTH-analogues on extinction of conditioned behavior. Prog. Brain Res., 3, 221-235.

- Whishaw, I.Q. (1985). Cholinergic receptor blockade in the rat impairs locale but not taxon strategies for pllace navigation in a swimming pool. Behav. Neurosci, 99, 979-1005.

- Whishaw, 1.Q. (1987). Hippocampal, granulle cell and CA3-4 lesions impair formation af a place learning-set in the ral and induce reflex epilepsy. Behav. Brain Res., 24, $59-72$.

- Whishaw, I.Q. \& Mittleman, G. (1986). Visits to starts, routes, and places by rats (Ratlus norvegicus in swimming pool navigation tasks. J. Contp. Psychol. 100, 422-431.

- Whishaw, 1.Q., OConner, W.T. \& Dunnett, S.B. (1985). Disruption of central cholinergic mechanisms in the rat by basal forebrain lesion or atropine: effects on feeding, sensorimotor behaviour, locomotor activity and spatial nawigation. Behut. Brain Res, 17, 103-115.

- Whitehouse, P.I., Price, D.L., Clark, A.W. Coyle, J.T, Delong, M.R. (1981). Alzheimer's disease: evidence for selective loss of cholinergic neurons in the nucleus basalis. Ann. Neurol, 10, $122-126$.

- Willians, R.A. Campbell, B.A. (1961). Weight loss and quinine-milk ingestion as measures of "hunger" in infant and adult rats. J. Comp. Physiol. Psychol., 54, 220-222.

- Willig, F., Palacios, A., Monmaur, P., M'Harzi, M., Laurent, J. \& Delacour, ]. (1987). Short-term memory, exploration and motor activity in aged rats. Neurobiol. Aging, 8, 393-402.

- Willner, P. Wise, D., and Ellis, T. (1986). Specific disruption of spatial behaviour in rats by central muscarinic receptor blockade. Psychopharmacology, 90, 29-235.

- Winer, B.J. Statistical principles in experimental design, MoGraw-Hill Book Company: New 
York, 1971.

- Winocur, G. (1988). Long-term memory loss in senescent rats: Neuropsychological analysis of interference and context effects. Psychol. Aging, 3, 273-279.

- Witherspoon, D. \& Moscovitch, M. (1989). Stochastic independence between two implicit memory tasks. I. Exp. Psychol.: Leam. Mem. Cogn., 15, 22-30.

- Wree, A., Kaever, C., Birgel, B., Schleicher, A. Horwath, E. \& Zilles, K. (1991). Local cerebral glucose utilization in the brain of old, learning impaired rats. Histochemisty $y, 95,591-603$.

- Yourg, S.I. \& Kamil, A.C. (1982). Response strategies in the radial arm maze: Running around in circles. Anim. Leam. Behav., 10, 530-534.

- Zacks, R.T. (1982). Encoding strategies use by young and elderly adults in a keeping track task. J. Gerontol., 37, 203-211.

- Zeldin, R.K., \& Olton, D.S. (1986). Rats acquire spatial learning sets. J. Exp. Psychol.: Anim.

Behav. Proces., 12, 412-419. 



\section{SAMENVATTING EN CONCLUSIES}

\section{Introductie}

Naarmate de leeftijd toeneemt zullen de cognitieve vermogens van de meeste mensen af́nemen. In toenemende mate ervaren mensen dat problemen met het ophalen van informatie uit het geheugen een negatieve invloed heeft op hun functioneren en dientengevolge de subjectieve qualiteit van het leven negatief beinvloed wordt. De verslechtering in cognitief functioneren is dramatischer bij mensen die leiden aan dementie, wat gezien kan worden als een pathologische vorm van het verouderingsproces. Omdat de levensverwachting var de mens in de komende decennia zal toenemen, zullen, absoluut gezien, meer mensen geconfronteerd worden met de problemen en ziekten die gepaard gaan met veroudering (dubbele vergrijzing). Een beter inzicht in de processen die ten grondslag liggen aan dementie en aan de leeftijdsgerelateerde afname in cognitieve functies zou kunnen leiden tot medische behandelingen, en diagnostische hulpmiddelen die de verslechtering in prestaties vroegtijdig kunnen onderkennen. Daaremboven kunnen risicofactoren worden onderkend die een negatieve invloed hebben op de cognitieve functies op latere leeftijd. Als gevolg van dit onderzoek zou de qualiteit van het leven op oudere leeftijd verbeterd kunnen worden. Ofschoon humane studies karakteristieken van de cognitieve veroudering hebben kunnen onderkennen, heeft het humane onderzoek zijn practische en ethische beperkingen.

De achteruitgang in cognitieve functies die gepaard gaan met veroudering en dementie is onderzocht in experimenten waarin gebruik gemaakt werd van dieren aangezien de practische en ethische beperkingen in mindere mate aanwezig zijn. Aangenomen wordt dat, indien adequate diermodellen worden gebruikt, de resultaten van experimenten met dieren geëxtrapoleerd kunnen worden naar de mens. In het laatste decennium is er veel aandacht besteed aan de ontwikkeling van valide diermodellen voor de afname in cognitieve functies bij veroudering en dementie. Dit onderzoek heeft laten zien dat de leefijdsgerelateerde afname in cognitieve prestaties zoals die bij dieren worden gevonden in grote mate overeenkomen met de karakteristieken zoals die bij de mens worden gevonden. De interne validiteit van de diermodellen, maar ook van de humane studies, kan echter laag zijn. In deze dissertatie werd een diermodel van cognitieve veroudering nader onderzocht. De prestatie van ratten van verschillende leeftijden werd gemeten in verschillende leer- en geheugentaken, gebruikmakend van verschillende testprocedures in deze taken. Daarenboven werd nagegaan in hoeverre er leeftijdsgerelateerde verschillen waren in niet-cognitieve gedragingen. Leeftijdsgerelateerde verschillen in deze niet-cognitieve gedragingen zouden kunnen leiden tot leeftijdsgerelateerde prestatieverschillen in leer- en geheugentaken. Door gebruik te maken van én rattestam en het meten van één dimensie van leren en geheugen, zouden de studies beschreven in deze dissertatie een beter inzicht kunnen verschaffen in de 
aard van de leeftijdsgerelateerde achteruitgang in prestatie in leer- en geheugentaken. Op zijn beurt zou dit meer informatie kunnen opleveren met betrekking tot de validiteit van de gebruikte diermodellen van cognitieve verouderingen.

In deze dissertatie werd ook een diermodel geïntroduceerd waarin een fundamenteel neurochemisch proces, het gebruik van glucose in zenuwcellen, was aangedaan. Er is verondersteld dat een afname in de mogelijkheid van zenuwcellen om over glucose te beschikken ten grondslag kan liggen aan de ziekte van Alzheimer. Nagegaan werd welke gedragsmatige effecten gevonden konden worden in een diermodel voor cognitieve veroudering na verstoring van het glucoseverbruik in het centrale zenuwstelsel. In de laatste twee hoofdstukken van de dissertatie werden de gedragsmatige effecten van een noötropicum bestudeerd. Noötropica zijn stoffen die verondersteld worden de leeftijdsgerelateerde achiteruitgang in cognitieve functies, en zoals die gevonden worden bij dementie, tegen te gaan. In eén studie werd het effect van het noötropicum op emotionele reactiviteit bestudeerd. In een tweede studie werd nagegaan welke effecten een chronische behandeling met het noötropicum had op spatieel leren. Tevens werd in de tweede studie bestudeerd of het noötropicum de beschikbaarheid van alternatieve energie reserves verhoogde, hetgeen inzicht zou kunnen verschaffen in het werkingsmechanisme van het noötropicum.

\section{Samenvatting van de studies}

Hoofdstuk 2:

In de eerste studie werd de ontwikkeling van een spatiële discriminatie leertaak, het conusveld, beschreven. Het conusveld biedt de mogelijkheid om tegelijkertijd twee aspecten van leren en geheugen te meten, i.e. werkgeheugen en referentiegeheugen. Bovendien kunnen in een conusveldtaak ook aspecten van gedrag gemeten worden (e.g. een systematische manier van het ophalen van het voedsel) die zouden kunnen interfereren met de leer- en geheugenprestatie en als gevolg daarvan tot een onjuiste interpretatie wan de resultaten zou kunnen leiden. Het conusveld werd ook beschouwd als een waardevolle taak voor onderzoek naar de leeftijdsgerelateerdle verslechtering in cognitieve functies omdat gecontroleerd kon worden voor verschillende confounders (snelheid van responderen, motivatie, en een systematiek in het ophalen van het voedsel). Verschillende experimenten werden beschreven waarin een consistent leeftijdsgerelateerde verslechtering in prestatie werd gevonden voor zowel werk- als referentiegeheugen. Eén van de experimenten toonde aan dat het conusveld een geschikte taak is voor het uitvoeren van longitudinale studies. Samengevat blijkt het conusveld een geschikt apparaat voor het onderzoek naar de leeftijdsgerelateerde afname in cognitieve functies.

Hoofdstuk 3 :

In de tweede studie werden Lewis ratten van 5 verschillende leeftijden $(4,12$, 
18,24 en 30 maanden oud) getest in het conusveld. Het doel van de studie was om na te gaan hoe het verloop van de verslechtering van de cognitieve functies was over verschillende tijdpunten van de levensloop van de rat. Onverwachts werd gevonden dat de ratten van 18 maanden en ouder beter presteerden op de maat referentiegeheugen terwijl de 4 maanden oude ratten beter presteerden op de maat werkgeheugen dan de vier andere leeftijdsgroepen. Deze bevindingen kwamen niet overeen met de algemeen gevonden leeftijdsgerelateerde verslechtering in cognitieve prestaties. Omdat de 4 maanden oude ratten de beste

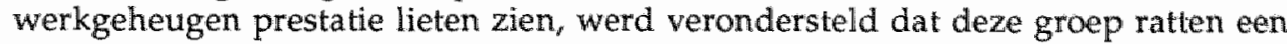
gewoonte ontwikkelden om niet beloonde conussen te blijven bezoeken. Deze gewoonte zou verklaard kunnen worden uit het feit dat alle conussen beloond waren tijdens de adaptatie trials. De ratten van de twee jongste leeftijdsgroepen (4 en 12 maanden oud) inspecteerden dus eigenlijk eerder beloonde conussen, hetgeen op zich een ecologisch voordeel zou kunnen hebben. De resultaten van de tweede studie ondersteunden de opvatting dat verschillende functies onafhankelijk van elkaar onderhavig zijn aan het verouderingsproces.

\section{Hoofdstuk 4:}

In de derde studie werden jonge en oude Lewis ratten getest in een eenvoudige conusveld taak. Het doel van deze studie was na te gaan welke oriëntatiestrategie jonge en oude ratten gebruikten in het conusveld. In deze studie werden de ratten gestart vanaf één positie en waren de beloonde conussen geclusterd in een hoek van het conusveld. In overeenstemming met andere studies werd er geen leeftijdsgerelateerde afname in prestatie gevonden in deze meer eenvoudiger spatiële discriminatie taak. De ratten konden bovendien ook gebruik maken van een egocentrische oriëntatiestrategie, een strategie waarvoor geen leeftijdsgerelateerde prestatieverschillen zijn gevonden. In probe-trials werden de ratten vanaf de tegenovergestelde startpositie opgestart en werd nagegaan welke conussen de ratten bezochten. De resultaten toonden aan dat zowel jonge als oude ratten een allocentrische oriëntatiestrategie gebruikten. Dientengevolge werd geconcludeerd dat het gebruik van een allocentrische oriëntatiestrategie niet noodzakelijker wijs leeftijdsafhankelijk is.

\section{Hoofdstuk 5:}

In de vierde studie werden Lewis ratten van verschillende leeftijden getest in een ander spatieel discriminatie paradigma. De ratten werden gestest in de Morris spatiële navigatie taak waarin een consistent leeftijdsgerelateerde prestatieverslechtering is gevonden. Omdat de oude Lewis ratten een relatief goede spatiële discriminatieprestatie vertoonden in het conusveld (zie Hoofdstuk 3 en 4), werd verondersteld dat er in de relatief gemakkelijke Morris taak geen leeftijdsgerelateerde prestatieverslechtering kon worden gevonden in deze stam. In tegenstelling tot de verwachtingen presteerden de oude Lewis ratten aanmerkelijk slechter dan jonge ratten en ratten van middelbare leeftijd. In een 'learning set' procedure werd gevonden dat oude ratten geen allocentrische orientatiestrategie gebruikten in de Morris talak. Dit werd 
geconcludeerd uit de bevinding dat de prestatie van de oude ratten niet veranderde als de spatiele cues veranderden. Verder werd in één experiment gevonden dat oude Lewis ratten in de Morris taak alleen een cue kuninen gebruiken als deze samenvalt met het ontsnappingsplatform. De resultaten suggeren dat de spatiële discriminatie prestatie van jonge en oude Lewis ratten taakafhankelijk is. Verondersteld werd dat dit te wijten is aan de verschillende operationalisaties van leerprestatie (ratio bezoeken aan beloonde en onbeloonde conussen in conusveld; ontsnappingstijd in Morris taak) en het verschil in 'reinforcers' (woedselbeloning in conusveld; ontsnapping aan water in Morris taak). De aversieve component van de Morris taak zou de leerprestatie van de oude dieren nadelig kunnen hebben beïnvloed.

\section{Hoofdistuk 6:}

De mogelijke leeftijdsafhankelijke verschillen in reactiviteit op stressvolle stimuli, zoals dat in Hoofdstuk 5 werd verondersteld, werd onderzocht in een vijfde studie. Lewis ratten en Brown-Norway ratten van 3 verschillende leeftijden werden onderworpen aan een open veld test. Analyse van twee geoperationaliseerde maten voor emotionele reactiviteit liet zien dat de gedragsmatige respons op het geconfronteerd worden aan een nieuwe omgeving bij jonge dieren anders was dan die bij ratten van middelbare leeftijd en oude ratten. Zoals verwacht bleek er tevens een stamverschil te zijn voor de twee geoperationaliseerde maten voor emotionele reactiviteit. In een tweede experiment werden dezelfde ratten die gebruikt waren in de open veld test, en jonge en oude Wistar ratten getest in een twee-weg actieve vermijdings taak. In deze shock-gemotiveerde leertaak werd geen consistente leeftijdsgerelateerde verslechtering gevonden. Opmerkelijk was zelfs dat oude Lewis ratten de taak sneller leerden dan de jonge Lewis ratten. Conform andere studies, bleek dat de prestatie in deze taak stamafhankeliik was. Omdat de leerprestatie in de actieve vermijdingstaak grotendeels afhankelijk is van de reactiviteit op de aversieve stimulus (shock), werd aangenomen dat er leeftijdsgerelateerde en stamathankelijke verschillen bestaan voor de gedragsmatige respons op stressvolle stimuli. De resultaten van deze studie suggereren dat leeftijdsgerelateerde verschillen in de reactiviteit op stressvolle stimuli de interpretatie van leeftijdsgerelateerde verschillen in leer- en geheugenprestatie, zoals die gemeten worden in leertaken met een aversieve component (water, shock en 'novelty'), bemoeilijken.

\section{Hoofdstuk 7:}

Een andere factor die zou kunnen bijdragen aan de leeftijdsgerelateerde verschillen in leerprestatie is een leeftijdsgerelateerde verandering in motivatie om te presteren. Een veel voorkomende wijze om dieren te motiveren is het voedseldepriveren van dieren en het gewenste gedrag te belonen met voer. Oude dieren wegen echter zwaarder dan jonge dieren en het is daarom voorstelbaar dat voedseldeprivatie bij jonge en oude dieren een verschillend effect op de motivatie om te presteren bewerkstelligt. Een manier om de 
motivatie van jonge en oude dieren zoveel mogelijk gelijk te krijgen is door het gewicht van oude ratten percentueel verder te reduderen dan dat van jonge ratten ( $100 \%$ is het gewicht als voedsel altijd voorradig was). Deze methode is benoemd als een differentiële deprivatie techniek. In de zesde studie werden Brown-Norway ratten van verschillende leeftijden getest in een loopgang, twee maal op twee verschillende depriwatieniveaus. De motivatie om voer te verkrijgen werd tevens eenmaal, tijdens het laagste deprivatieniveau, gemeten in een operante conditionerings kamer waar de ratten werden getest op een progressive ratio schedule of reinforcement'. De resultaten van deze studie lieten zien dat het differentieel depriveren van ratten van verschillende leeftijden als een methode mag worden beschouwd die de motivatie van ratten van verschillende leeftijden vergelijkbaar maakt. Verder werd gevonden dat oude ratten meer training nodig hadden voordat de prestatie in de loopgang optimaal was. Dit betekent dat het bereiken van een optimale prestatie in voedselgemotiveerde leertaken verschillend kan zijn voor oude en jonge ratten. Als laatste bleek dat er geen relatie bestond tussen de geoprerationaliseerde maten voor motivatie in de loopgang en 'progressive ratio' test. Dit ondersteund het idee dat motivatie niet als één proces dient te worden gezien maar dat meerdere processen hierbij een rol spelen. Omdat verschillen in voedselmotivatie van invloed kunnen zijn op de leerprestatie, moet voor deze factor worden gecontroleerd, met name in verouderingsstudies.

\section{Hoofdstuk 8:}

In de zevende studie werd een "within subject design" gebruikt om veroudering te bestuderen. Jonge en oude ratten werden onderworpen aan verschillende cognitieve taken en niet-cognitieve tests om na te gaan welke relatie er bestond tussen de verschillende operationalisaties van gedrag. Daarenboven werd nagegaan of er een relatie bestond tussen de gedrags maten en drie biochemische parameters. Leeftijdsgerelateerde veranderingen in de relatie tussen de verschillende parameters zou informatie kunnen verschaffen over mogelijke verouderingsverschijnselen voor deze parameters. Er werd geen correlatie gevonden tussen de verschillende geoperationaliseerde gedragsmaten. Dit ondersteunt de opvatting dat het verouderen verschillend is voor (niet) cognitieve functies en dat dit individueel verschillend kan zijn. Voedselmotivatie en emotionele reactiviteit hadden voorspellingswaarde voor de prestatie in de verschillende leertaken. Echter het kan niet uitgesloten worden dat twee factoren de leerprestatie voor jonge en oude ratten differentieel beïnloeden. Hippocampale cholineacetyltransferaseactiviteit had geen voorspellende waarde voor de leerprestatie in jonge en oude ratten. Dit suggereert dat deze marker van cholinerge neurotransmissie niet gezien kan worden als een marker voor de cholinerge dysfunctie bij veroudering. Baseline bloedglucose niveau was gerelateerd aan de geoperationaliseerde maten voor leren in het conusveld (referentie- en werkgeheugen). Er werd verondersteld dat de leeftijdsgerelateerde afname in cognitieve functies gerelateerd zou kunnen zijn aan een verstoorde bloedglucose regulatie. 
Hoofdstuk 9:

De meeste diermodellen voor cognitieve veroudering en dementie hebben de rol van de cholinerge transmissie als uitgangspunt genomen. De functies van hel cholinerge systeem kan worden bestudeerd door lesies te maken in cholinerge kernen of het verstoren van de cholinerge neurtransmissie door middel van injecties van cholinerge antagonisten. In de achtste studie werd een diermodel gebruikt waarin de ratten behandeld werden met streptozotocine (intracerebroventriculair geinjecteerd). Streptozotocine verstoord de opname van glucose in neuronen, hetgeen verondersteld wordt ook ten grondslag te liggen bij de neurodegeneratieve verschijnselen zoals die voorkomen bij de ziekte van Alzheimer. Bij jonge streptozotocine behandelde ratten werd gevonden dat de prestatie op de inhibitoire vermijdingstaak verstoord was, maar dat de prestatie in de spatiële Morris taak niet verstoord was zoals dat gevonden wordt bij dieren die behandeld zijn met cholinerge antagonisten. Ratten van middelbare leeftijd bleken na streptozotocine behandeling wel een slechtere prestatie te hebben in de Morris taak. In de ratten van middelbare leeftijd bleek de hippocampale cholineacetyltransferaseactiviteit te zijn afgenomen. De afname in cholineacetyltransferaseactiviteit bij de ratten van middelbare leeftijd bleek te correleren met prestatie in de Morris taak. Deze correlatie werd niet gevonden in de controle ratten. De resultaten suggereren dat ratten van middelbare leeftijd behandeld met streptozotocine beschouwd kunnen worden als een model voor de cognitieve dysfuncties zoals die zich voordoen bij dementie. De resultaten geven aanleiding tot verdere bestudering van dit model op zowel gedragsmatig, biochemish en pathologisch niveau.

Hoofdstuk 10:

In de negende studie werd nagegaan wat het effect van een chronische behandeling met het noötropicum acetyl-l-carnitine op open veld gedrag in jonge en oude ratten. Acetyl-1-carnitine blijkt de leerprestatie van oud dieren te verbeteren en de discriminatievermogens bij ratten van middelbare leeftijd te verbeteren. De doelstelling van deze studie was na te gaan wat het effect van een behandeling met acetyl-1-carnitine was op een ander aspect van gedrag, en of dit verschillend was voor jonge en oude ratten. De oude behandelde ratten waren meer emotioneel reactief dan de oude controle ratten terwijl er geen effect van behandeling werd gevonden in jonge dieren. Ofschoon de resultaten geinterpreteerd werden als een behandeling geinduceerde toename in emotionele reactiviteit in oude ratten moet voorzichtigheid in acht genomen worden. De geoperationaliseerde maten van emotionaliteit zijn wellicht niet valide voor oude ratten (zie Hoofdstuk 6).

\section{Hoofdstuk 11:}

In de laatste studie werd het mogelijke werkingsmechanisme van het noötropicum acetyl-1-carnitine bestudeerd. Het begrijpen van het werkingsmechanisme zou van grote waarde zijn voor het ontwikkelen van cognitie verbeterende stoffen en mogelijke behandelingen voor ouderen en 
dementiepatiënten. Jonge en oude ratten werden chronisch behandeld met acetyl1-carnitine en getest in de Morris taak. Tijdens de training leerden de oude behandelde ratten sneller het platform te localiseren dan de onbehandelde oude ratten. De asymptotische prestatie was echter gelijk voor beide oude groepen oude ratten. Dit suggereert dat acetyl-1-carnitine het initiële leerprocess bij oude ratten versnelt, maar dat de behandeling geen effect heeft op het gebruik van een oplosstrategie. Nadat de ratten op asymptotisch niveau presteerden werd nagegaan welk effect werd gevonden na een intracerebroventriculaire injectie met cyanide. Cyanide veroorzaakt een kortdurende periode van histotoxische hypoxie. Er werd verondersteld dat acetyl-1-carnitine de beschikbaarheid over alternatieve energievoorraden zou toen toenemen en als gevolg daarvan de gevolgen van histotoxische hypoxie voor het neuronaal functioneren zou kunnen verminderen. De verminderde gevolgen voor het neuronaal functioneren, als gevolg van een acetyl-1-carnitine behandeling, werd bestudeerd door het meten van de ontsnappingstijd in de Morris taak. Na een dosis van 2.5 $\mu \mathrm{g}$ bleek de ontsnappingstijd van de ratten niet te zijn verhoogd. Dit zou kunnen betekerien dat deze dosis het neuronal functioneren niet zodanig beïnvloedt om gedragsmatige effecten te bewerkstelligen. Na een dosis van $5 \mu \mathrm{g}$ bleek echter dat de ontsnappingstijd 1 en 5 minuten na injectie was verhoogd voor jonge ratten. Chronische behandeling met acetyl-1-carnitine verminderde de door cyanide geïnduceerde gedragseffecten. De ontsnappingstijd van de oude ratten was niet verhoogd bij beide groepen. De afwezigheid van cyanide effecten bij oude dieren zou verklaard kunnen worden door een systematische verkeerde canule plaatsing. Een andere mogelijkheid zou echter kunnen zijn dat de injectie met cyanide de reeds slechte prestatie van de oude ratten niet verder zou kunnen verslechteren: om goed te presteren zouden oude ratten een allocentrische oriëntatie strategie moeten gebruiken, maar die oude Lewis ratten blijkbaar niet gebruiken in de Morris taak (zie Hoofdstuk 5). De resultaten doen vermoeden dat een chronische behandeling met acetyl-1-carnitine de beschikbaarheid van alternatieve energiebronnen van de cel vergroot en dientengevolge beter bestand is tegen energiearme periodes. Dit zou het werkingsmechanisme kunnen zijn van het noötropicum waardoor een verbetering van cognitieve functies kan optreden in oude ratten. Het paradigma dat werd gebruikt in deze studie zou bruikbaar kunnen zijn voor de bestudering van neuroprotectieve stoffen op zowel gedrags- en neurochemisch niveau.

\section{Concllusies}

In de hoofdstukken 2 tot en met 5 werd de prestatie van Lewis ratten van verschillende leeftijdsgroepen gemeten in twee verschillende spatiële discriminatie taken. Er werd geen consistent leeftijdsgerelateerde verslechtering in cognitieve prestaties gevonden. Deze bevindingen suggereren dat andere factoren dan leer- en geheugenprocessen bijdragen aan de leeftijdsgerelateerde verschillen in prestatie in de verschillende leer-en geheugentaken. Er werd aangenomen dat jonge Lewis ratten (tot en met 12 maanden) een gewoonte 
ontwikkelden om niet beloonde conussen te blijven bezoeken in het conusveld terwijl de oude Lewis ratten (vanaf 18-20 maanden) geen allocentrische oriëntatiestrategie gebruiken in de Morris taak. Door deze leeftijdsgerelateerde verschillen in oplosstrategiën is het niet mogelijk om leeftijdsgerelateerde verschillen in prestatie in leer- en geheugentaken, te interpreteren in termen van verschillen in leer- en geheugenprocessen. Naast de verschillen in oplosstrategiën zijn er nog meer factoren die kunnen bijdragen aan de leeftijdsgerelateerde verschillen in prestatie in leer-en geheugentaken.

In deze dissertatie zijn twee studies beschreven waarin leeftijdsgerelateerde verschillen in de reactiviteit op stressvolle stimuli en voedselmotivatie werden onderzocht. De resultaten van de studie waarin verschillen in reactiviteit op stresswolle stimuli werden gemeten suggereerden dat er een leeftijdsafhankelijk verschil was in de gedragsrespons op een aversieve stimulus. In de studie waarin de leeftijdsgerelateerde verschillen in voedselmotivatie werden bestudeerd werd aan de hand van de resultaten aangenomen dat een differentiële voedseldeprivatie techniek een goede manier is om het motivatieniveau van jonge en oude dieren vergelijkbaar te krijgen. De eerste zes studies geven aan dat het vrijwel onmogelijk is om verschillen in leer- en geheugenprestatie tussen jonge en oude dieren te interpreteren in termen van verschillen in leer-en geheugenprocessen. In de introductie (Hoofdstuk 1) werd al besproken dat het onmogelijk was om jonge en oude dieren met elkaar te vergelijken vanwege een definitie van leren. Verschillende auteurs hebben al gewezen op de mogelijke factoren die ten grondslag kunnen liggen aan de leeftijdsgerelateerde prestatieverschillen in leer- en geheugentaken (Ingram, 1985; Ray \& Barrett, 1973; Rigter, 1983; Sarter, 1987). Ofschoon de problemen onderkend worden, zijn er nog steeds veel studies die concluderen dat er leeftijdsgerelateerde verschillen in leren en geheugen zijn. Voordat een dergelijke conclusie kan worden getrokken zou men zich eerst moeten vergewissen van het feit dat voor alle mogelijke interfererende variabelen gecontroleerd is.

Gezien de resultaten van de eerste zes studies, waarin voornamelijk één rattestam (Lewis) werd gebruikt, werd geconcludeerd dat de leeftijdsgerelateerde prestatieverschillen taakafhankelìjk was en dat daarom de verschillen geen verschillen leer- en geheugencapaciteit kunnen weergeven. Door verschillende taken en verschillende procedures te gebruiken kan men nagaan wat de aard kan zijn van de leeftijdsgerelateerde prestatieverschillen. Ook de bestudering van niet-cognitieve processen in ratten van verschillende leeftijden kan meer inzicht geven over de aard van de leeftijdsgerelateerde achteruitgang in prestatie in leeren geheugentaken. Dit leidt tot de eerste conclusie:

Leeftijdsverschillen in leer. en geheugenprestatie dienen bestudeerd te worden in verschillende taken gebruikmakend oan verschillende procedures. Daarenboven dient het effect van leeftijd bestudeerd te worden in niet-cognitieve tests. Conclusies ten aanzien van leeftijdsgerelateerde achteruitgang in leer- en geheugenprocessen moeten de resultaten van de verschillende 
studies in acht nemen.

Gebruikmakend van deze methode zou men tot de conclusie kunnen komen dat het niet mogelijk is om leer-en geheugenprocessen te vergelijken voor jonge en oude ratten. Dit is in deze dissertatie duidelijk gedemonstreerd voor de Lewis stam.

Dit zou weer aanleiding kunnen zijn om te concluderen dat er geen middelen zijn om leer- en geheugenprocessen bij jonge en oude ratten te vergelijken. Men zou zich echter kunnen afvragen of het wel noodzakelijk is om leeftijdsgerelateerde verschillen in leren en geheugen te onderzoeken. De primaire doelstelling van het verouderingsonderzoek is om het verouderingsproces te begrijpen en de mogelijke oorzaken voor het verval in cognitieve functies te achterhalen. Naar aanleiding van de resultaten van de eerste zes studies kon geconcludeerd worden dat er leeftijdsgerelateerde verschillen waren in niet-cognitieve gedrag, en dat het aannemelijk was dat deze factoren interfereerden met leer-en geheugen prestatie. Dientengevolge kan het begrijpen van de leeftijdsgerelateerde veranderingen in niet-cognitief gedrag leiden tot en beter inzicht in de leeftijdsgerelateerde veranderingen in cognitief gedrag.

Verder dient nog opgemerkt te worden dat leren/geheugen een concept is dat niet één proces weerspiegelt. Meerdere processen dragen bij aan de adaptieve verandering in het gedrag. Tot op heden is er weinig aandacht besteed aan cognitieve processen die ten grondslag kunnen liggen aan de leeftijdsgerelateerde verschillen in leer- en geheugenprestaties. Onlangs werd gerapporteerd dat er een leeftijdsgerelateerde verandering is in het gebruiken van een oplosstrategie (Caprioli et al, 1991), maar dat de prestatie van de ratten onafhankelijk is van de gebruikte oplosstrategie. Omdat een gelijksoortige leeftijdsgerelateerde verandering in het gebruik van een oplosstrategie ook is gevonden bij mensen (Zacks, 1982), zouden experimenten waarin de leeftijdsgerelateerde veranderingen in het gebruik van oplosstrategiën interessante resultaten opleveren die vergelijkbaar zijn met de humane studies.

Een ander cognitief proces dat onderkend is onderhavig te zijn aan verval in de ouder wordende mens (i.e, aandacht; Giambra Quilter, 1988; Stankov, 1988; Plude \& Doussard-Roosevelt, 1988), heeft nog geen aandacht gekregen in het dierexperimentele verouderingsonderzoek. De voordelen van het onderzoek met dieren zou van groot voordeel kunnen zijn in het bestuderen van leeftijdsgerelateerde afname in aandachtsprocessen. De bestudering van afzonderlijke cognitieve processen zou kunnen leiden tot een beter inzicht in de leeftijdsgerelateerde achteruitgang in prestatie in leer-en geheugentaken. Dit leidt tot een tweede conclusie:

Onderzoek dat de achteruitgang in cognitieve functies bestudeerd bij veroudering en dementie zou ook aandacht moeten besteden aan cognitieve processen die ten grondslag zouden kunnen liggen aan de leeftijdsgerelateerde prestatieverschillen in leer- en 
geheugentaken.

Een andere benadering die meer inzicht zou kunnen verschaffen in verouderingsprocessen is het gebruik van een within-subject design. De relatie tussen verschillende parameters wan leren, niet-cognitief gedrag en biochemische maten verschaft informatie over de leeftijdsgerelateerde veranderingen in de relatie tussen parameters. Zo heeft men aangetoond dat de integriteit van de cholinerge cellen in de basale voorhersenkernen nodig is voor spatieel discriminatie leren (Fischer, et al., 1991), de duur van bouts gedurende de paradoxale slaap gerelateerd is an cognitieve functies (Markowska et al., 1988), centraal glucoseverbruik is gerelateerd is aan spatieel leren (Gage et al., 1984b), en bloedglucose is gerelateerd aan spatieel leren in oude ratten (zie Hoofdstuk 8). Studies waarin verschillende aspecten van gedrag en biochemie worden gecorreleerd zouden meer inzicht kunnen verschaffen in de relatie tussen de afname in functies met het ouder worden. Een derde conclusie is:

Correlationele studies kunnen meer inzicht verschaffen in de relatie tussen gedrag en biochemie en als gevolg een significante bijdrage kunnen leveren in het onderzoek nat verouderingsprocessen.

In Hoofdstuk 9 werd gevonden dat dat een afname in de beschikbaarheid van glucose in de hersenen de prestatie van ratten wan middelbare leeftijd deed verslechteren in de spatiële Morris taak. De prestatie in de Morris taak bleek gerelateerd te zijn aan de hippocampale cholinerge activiteit. Deze bevindingen suggereren dat een fundamenteel biochemisch proces ten grondslag ligt aan de afname in cholinerge en cognitieve functies. Dit zou kunnnen verklaren dat behandelingen met cholinerge medicamenten niet geleid hebben tot een betrouwbare behandelingsmethode. Er is gevonden dat een afname van het verbruik van glucose in de hersenen (energie metabolisme) gerelateerd is aan de afname in cognitieve functies (Gage et al., 1984). Het zou dus beargumenteerd kunnen worden dat een verhoging van de energetische parameters zou kunnen leiden tot een verbetering van cognitieve functies in de ouder wordende mens en dementie patiënten. In de laatste twee studies werden de gedragsmatige effecten van een noötropicum, acetyl-l-carnitine, bestudeerd. De werking van dit noötropicum zou kunnen berusten op een verhoging van de energetische parameters van neuronen en zou als gevolg daarvan de neuronale functies kunnen behouden/verbeteren. Verschillende studies hebben laten zien dat acetyl-1-carnitine de leer- en geheugenprestatie van oude ratten verbeterd (Caprioli et al, 1990; Ghirardi et al., in press). Gezien de resultaten uit de laatste studie, waarin het cyanide geinduceerde verstoorde gedrag minder dramatisch was na een behandeling met acetyl-l-carnitine, werd geconcludeerd dat het werkingsmechanisme van het noötropicum gerelateerd is aan de energiestatus van neuronen. De bevindingen dat een afname in het energieniveau van de hersenen een onderliggend proces zou kunnen zijn aan de verschijnselen zoals 
die zich woordoen bij veroudering, en dat een stof die de energetische parameters van neuronen verhoogd een positief effect heeft op cognitieve functies, rechtvaardigen verder onderzoek die de functie van de basale processer in relatie tot het energiemetabolisme van neuronen bestudeerd. De vierde conclusie is:

De bestudering van fundamentele processen die een effect hebben op de energiehuishouding van het brein kumnen een significante bijdrage leveren in het onderzoek naar verouderingsprocessen en zou dientengevolge kunnen leiden tot een effectieve behandeling van cognitieve dysfuncties als gevolg van (pathologisch) verouderen.

\section{Samenvatting van conclusies}

De leeftijdsgerelateerde afname in leer- en geheugenprestaties weerspiegelen niet altijd leeftijdsgerelateerde verschillen in leer- en geheugenprocessen. Door het gebruik van een rattestam en het bestuderen van de prestatie in verschillende modellen voor cognitieve veroudering, die én dimensie van leren/geheugen meten, kan men een beter inzicht krijgen in de aard van de leeftijdsgerelateerde achteruitgang in cognitieve prestaties. Ook zal de bestudering van (niet)cognitieve processen, die de leerprestatie kunnen beïnvloeden, meer inzicht geven over de aard van het cognitieve verval. Correlationele studies waarin zowel gedrags- als biochemische variabelen worden bestudeerd, kunnen een significante bijdrage leveren in het onderzoek naar cognitieve veroudering. Als laatste zou de bestudering van gedrag en biochemie na een interventie die de fundamentele processen aandoet een vruchtbare benadering kunnen zijn in het verouderingsonderzoek hetgeen op zijn beurt weer zou kunnen leiden tot een effectieve behandeling van cognitieve dysfuncties als gevolg van (pathologisch) verouderen. 



\section{Woorden van dank}

Zoals all door veel promovendi kenbaar is gemaakt, is de totstandkoming van een proefschrift het werk van vele mensen. Daarom zou ik op deze pagina eigerlijk alle mensen willen noemen die mij geholpen hebben bij de totstandkoming van dit proefschrift. $\mathrm{k}$ ben echter van mening dat dit een ondoenlijke zaak is omdat er heel veel mensen hebben bijgedragen aan de totstandkoming van dit proefschrift (zelfs met een lettergrootte 9 zou deze pagina nog te klein zijn). Ik zou me daarom willen beperken tot de groepen van mensen die ik dank verschuldigd ben.

De familie:

De familie zou ik willen bedanken voor de steun waarop ik kon rekenen als ik die nodig had.

De onderzoeksgroep:

Ik zou alle medewerkers van de vakgroep Neuropsychologie \& Psychobiologie, maar ook enkele medewerkers van de vakgroep Fysiologie (thans

Bewegingswetenschappen) en de Instrumentele Dienst, willen bedanken die een bijdrage hebben geleverd aan het onderzoek, en diegenen die zorgden voor een prettige sfeer op de afdeling.

De vriendenkring:

Ook zij hebben mij, een ieder op zijn eigen wijze, gestimuleerd om het proefschrift tot een goed einde te brengen.

De sportorienden:

De mensen van de 'Cheetahs', 'Babbelboks' en 'Shantung-siau-lim' zou ik willen bedanken voor de sportieve uurtjes waarin ik veel ontspanning heb kunnen vinden, ofschoon er mensen waren die niet het idee hadden dat ik mij aan het ontspannen was.

Nogmaals, dank je wel! 



\section{Curriculum Vitae}

De schrijver van dit proefschrift werd geboren op 30 januari 1962 te Heerlen. In 1980 werd het HAVO diploma behald en werd het VWO diploma behaald in 1982. Daarna werd aangevangen met de studie Psychologie aan de Katholieke Universiteit van Nijmegen, en werd het propadeutisch examen behaald in 1983. In 1988 werd het doctoraalexamen behaald (afstudeerrichting Vergelijkende en Fysiologische Psychologie). Gedurende twee perioden (november 1988 - juni 1989 en juli 1990 - juli 1992) werd onderzoek verricht bij de Rijksuniversiteit Limburg (Vakgroep Neuropsychologie \& Psychobiologie, hoofd prof. dr. J. Jolles) waarwan een groot gedeelte in dit proefschrift is beschreven. 



\section{LIST OF PUBLICATIONS}

\section{PAPERS}

- Blokland, A., Honig, W., \& Raaijmakers, W. (in press) The effect of intra-hippocampal scopolamine injections in a repeated spatial acquiistion task. Psychopharmacology.

- Blokland, A., Raajinakers, W.G.M., Honig, W. \& Van der Staay, F.J. (1992). Spatial discrimination learning and orientation strakegy in young and old Lewis rats. Neuroscience Research Communications, $10,105-110$.

- Blokland, A., Prickaerts, I. \& Raaijmakers, W.G.M. (1992). Reduced level of anxiety in adult Lewis rats after chronic ethanol consumption. Physiology \& Beha vior, $51,245-248$.

- Blokiand, A., Raaijmakers, W., Van der Staay, F.J., Jolles, J. (1990). Differential effect of Acetyl-L-Carritine on open field behavior in young and old rats. Physiology \& Behaviot, 47, 783785.

- Raaijmakers, W.G.M., Blokland, A, Van der Staay, F.J, \& Jolles, J. (1990). Differental behavioral effects of chronic oral administration of Acetyl L-Camitine in young and old rats. In: C.F.A. van Bezooijen, R. Ravid, \& A.A.J. Verhofstad (Eds.), From gene to man. Rijswijk: Stichting Gerontologie en Geriatrie, pp. 173-177.

- Raaijmakers, W.G.M., Van der Staay, F. , Drinkenburg, W.H.I.M. \& Blokland, A. (1990). Age differences and effects of lesion in the nucleus basalis magnocellularis on a seven choice task in a radiall alley maze. In: C.F.A. van Bezooijen, R. Ravid, \& A.A.J. Verhofstad (Eds.), From gene to man. Rijswijk: Stichting Gerontologie en Geriatrie, pp. 159-163.

- Van der Staay, F.J., Blokland, A., \& Raaijmakers, W. (1990). Different time course for age-related changes of behavior in a complex spatial cone-field discrimination task in Lewis rats.

Psychobiology, 18, 305-311.

- Van der Staay, F.J., Krechting, B., Blokland, A., Raaijmakers, W.G.M. (1990). The cone field: a spatial discrimination task for the automatic and simultaneous assessment of working and reference memory in rats. Journal of Neuroscience Methods, 31, 13-22.

- Van der Staay, F.J, Raaijmakers, W.G.M., \& Blokland, A. (1990). Age differences in spatial memory of rats in two tasks differing only for the response required to find and obtain the food reward. In: C.F.A. van Bezooijen, R. Ravid, \& A.A.J. Verhofstad (Eds.), From gene to man. Rijswijk: Stichting Gerontologie en Geriatrie, pp. 154-158.

\section{PAPERS SUBMITTED}

- Blokland, A. Correlation between behavioral and biochemical parameters in young and old Lewis rats. submitted to Behavioral and Neural Biology

- Blokland, A, Honig, W. \& Jolles, J. Neuroprotective effect of acetyl-l-camitine in a new paradigm for behavioral assessment during acute metabolic infibition. submitted to Pharmacology Biochemistry \& Behavior.

- Blokland, A. Honig. W. Raaimakers, W. Age-related changes in spatial discrimination performance in Lewis rats. revised version submitted to Behavioral Neuroscience.

- Blokland, A. \& Jolles, I. Spatial learning deficit and reduced hippocampal ChA activity after

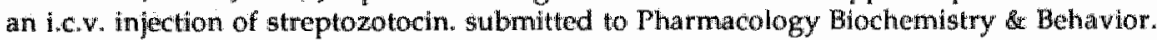

- Blokland, A., Prickaerts, J. \& Raaijmakers, W. Spatial and temponal discrimination learning is not affected after chronic ethanol treatment in Lewis rats. revised version submitted to Pharmacology Biochemistry \& Behawior.

- Blokland A., Raaijmakers, W., van der Staay, F.J. Agewelated changes in the response to stressful stimuli in different rat strains. revised version submilted to Behawioural Brain Research. - Blokland, A., Radijmakers, W. Age differences in food motivation in Brown-Norway rats. submitted to Learning and Motivation

\section{ABSTRACTS}

- Blokland, A., Drinkenburg, W.H.I.M., Van der Staay, F.J., Willems-van Bree, E, Waanders, R., 
Tonnaer, J.A.D.M. Ratjmakers, W.C.M. (1987). Chronic treatment with ORG 2766 after basalis lestons in old rats: Effects on spatial discrimination and cortical choline acetyltransferase. 29. TeaP (Tagung experimentell arbeitender Psychologen). April 12-16, Institut für Psychologie der RWTH Aachen, Federal Republic of Germany, Abstract no. 16.

- Idem in: Proceedings of the Eight Low Countries Meeting. May 22 1987, Nijmegen, The

Metherlands. p. 12.

- Blokland, A, Drinkenburg, W., Van der Staay, Fy., Willems-van Bree, E, Waanders, R., Tonnaer, I., \& Raijmakers, W.G.M. (1987). Effects of chronic treatment with ORG 2766 in old rats with nucleus basalis lesions on spatial discrimination and cortical choline acetyltransferase. Proceedings of the 28th Dutch Federation Meeting. Dutch Federation- Federation of medical scientific societies, April 22-23, Nijmegen, The Netherlands, Abstract no. 50.

- Blokland, A., Prickacrts, I, \& Raaijmakers, W. (1991). Spatial and temporal discrimination learning is not affected after chronic ethanol treatment in Lewis rats. Retraite NWOdeelwerkgemeenschap gerontologie en geriatrie, 24-25 Oktober, Egmond aan Zee.

- Blokland, A. Raaijmakers, W, \& Van der Staay, F.J. (1989). Age differences in reference memory of Lewis rats are compromised by more extensive scanning of the test environment by younger rats. Proceedings of the 10th Low Country Meeting, May 26, Namur, Belgium. p. 70.

- Blokland, A.. Raaijmakers, W., \& Jolles, J. (1992). Spatial learning deficit and reduced hippocampal ChAT activity after an i.c.v. injection of streptozotocin in rats: An animal model for the cogntive decline in dementia ? Proceedings of the 33rd Dutch Federation Meeting. Dutch Federation- Federation of medical scientific societies, April 21-22, Rotterdam, The Netherlands. - Idem in: Archives Internationale de Pharmacodynamie (in press).

- Blokland, A. Honig, W., \& Raaijmakers, W. (1991). Effects of intra-hippocampal scopolamine injections in repeated spatial acquisition. Proceedings of the Twelfth Low Countries Meeting. May 23-24, Cologne, Federal Republic of Germany, p. 44.

- idem in: ESF Winter School, Organisation and plasticity of neural systems in learning and memory, January 4-11, 1992, Zuoz, Switzerland.

- Drinkenburg, W.H.I.M., Blokland, A., Van der Staay, F. J., Willems van Bree, E., Waanders, R, Tonnaer, J.A.D.M., \& Raaijmakers, W.G.M. (1987). Influence of age and nucleus basalis lesions on spatial discrimination learning and cortical choline acetyltransferase activity in rats. 29. TeaP (Tagung experimentell arbeitender Psychologen). April 12-16, Institut für Psychologie der RWTH Aachen, Federal Republic of Germany, Abstract no. 61.

- idem in: Proceedings of the Eight Low Countries Meeting. May 22 1987, Nijmegen, The Netherlands. p. 16.

- Drinkenburg, W.H.I.M., Blokland, A., Van der Staay, F.J., Willems-van Bree, E., Waanders, R, Tommaer, J.A.D.M. \& Raaijmakers, W.G.M. (1987). Effects of nucleus basalis lesions and age on spatial discrimination learning and cortical choline acetyltransferase in rats. Proceedings of the 28th Dutch Federation Meeting. Dutch Federation-Federation of medical scientific societies, April 22-23, Nijmegen, The Netherlands, Abstract mo. 131.

- Prickaerts, $J$. Blokland, A., Raaimakers (1991). Reduced level of anxiety in adult Lewis rats after chronic ethanol consumption. Proceedings of the Twelfth Low Countries Meeting. May 23-24, Cologne, Federal Republic of Germany, p. 73.

- Raaijmakers, W. \& Blokland, A. (1991). Age-related impairments in memory performance of rats; do they reflect true age differences in memory? Retraite NWO-deelwerkgenteenschap gerontologie en geriatrie, 24-25 Oktober, Eggmond aan Zee.

- Raaijmakers, W. Blokland, A. \& van der Staay. F.J. (1992). Age-related impairments in memory performance of rats; do they reflect true age differences in memory? Proceedings of the 10th Low Country Mecting. May 26, Namur, Belgium. p. 9. 